\title{
Wind and Wake Sensing with UAV Formation Flight: System Development and Flight Testing
}

Trenton Jameson Larrabee

West Virginia University

Follow this and additional works at: https://researchrepository.wvu.edu/etd

\section{Recommended Citation}

Larrabee, Trenton Jameson, "Wind and Wake Sensing with UAV Formation Flight: System Development and Flight Testing" (2013). Graduate Theses, Dissertations, and Problem Reports. 678.

https://researchrepository.wvu.edu/etd/678

This Thesis is protected by copyright and/or related rights. It has been brought to you by the The Research Repository @ WVU with permission from the rights-holder(s). You are free to use this Thesis in any way that is permitted by the copyright and related rights legislation that applies to your use. For other uses you must obtain permission from the rights-holder(s) directly, unless additional rights are indicated by a Creative Commons license in the record and/ or on the work itself. This Thesis has been accepted for inclusion in WVU Graduate Theses, Dissertations, and Problem Reports collection by an authorized administrator of The Research Repository @ WVU. For more information, please contact researchrepository@mail.wvu.edu. 


\title{
Wind and Wake Sensing with UAV Formation Flight: System Development and Flight Testing
}

\author{
Trenton Jameson Larrabee
}

Thesis submitted

\begin{abstract}
to the Benjamin M. Statler College of Engineering and Mineral Resources at West Virginia University
\end{abstract}

\author{
in partial fulfillment of the requirements for the degree of \\ Master of Science in \\ Aerospace Engineering \\ Marcello Napolitano, Ph.D., Chair \\ Yu Gu, Ph.D. \\ Haiyang Chao, Ph.D. \\ Department of Mechanical and Aerospace Engineering \\ Morgantown, West Virginia \\ 2013
}

Keywords: Wind Estimation, Wind Sensing, Wake Modeling, Wake Sensing, Wake Visualization, Ground Control Station, Unmanned Aircraft System, Formation Flight, Unscented Kalman Filter 


\section{Abstract \\ Wind and Wake Sensing with UAV Formation Flight: System Development and Flight Testing Trenton Jameson Larrabee}

Wind turbulence including atmospheric turbulence and wake turbulence has been widely investigated; however, only recently has it become possible to use Unmanned Aerial Vehicles (UAVs) as a validation tool for research in this area. Wind can be a major contributing factor of adverse weather for aircraft. More importantly, it is an even greater risk towards UAVs because of their small size and weight. Being able to estimate wind fields and gusts can potentially provide substantial benefits for both unmanned and manned aviation. Possible applications include gust suppression for improving handling qualities, a better warning system for high wind encounters, and enhanced control for small UAVs during flight. On the other hand, the existence of wind can be advantageous since it can lead to fuel savings and longer duration flights through dynamic soaring or thermal soaring.

Wakes are an effect of the lift distribution across an aircraft's wing or tail. Wakes can cause substantial disturbances when multiple aircraft are moving through the same airspace. In fact, the perils from an aircraft flying through the wake of another aircraft is a leading cause of the delay between takeoff times at airports. Similar to wind, though, wakes can be useful for energy harvesting and increasing an aircraft's endurance when flying in formation which can be a great advantage to UAVs because they are often limited in flight time due to small payload capacity.

Formation flight can most often be seen in manned aircraft but can be adopted for use with unmanned systems. Autonomous flight is needed for flying in the "sweet spot" of the generated wakes for energy harvesting as well as for thermal soaring during long duration flights. For the research presented here formation flight was implemented for the study of wake sensing and gust alleviation.

The major contributions of this research are in the areas of a novel technique to estimate wind using an Unscented Kalman filter and experimental wake sensing data using UAVs in formation flight. This has been achieved and well documented before in manned aircraft but very little work has been done on UAV wake sensing especially during flight testing. This document describes the development and flight testing of small unmanned aerial system (UAS) for wind and wake sensing purpose including a Ground Control Station (GCS) and UAVs.

This research can be stated in four major components. Firstly, formation flight was obtained by integrating a formation flight controller on the WVU Phastball research UAV aircraft platform from the Flight Control Systems Laboratory (FCSL) at West Virginia University (WVU). Second, a new approach to wind estimation using an Unscented Kalman filter (UKF) is discussed along with results from flight data. Third, wake modeling within a simulator and wake sensing during formation flight is shown. Finally, experimental results are used to discuss the "sweet spot" for energy harvesting in formation flight, a novel approach to cooperative wind estimation, and gust suppression control for a follower aircraft in formation flight. 


\section{Acknowledgments}

I would like to thank my committee chairman Dr. Marcello R. Napolitano and my committee members Dr. Haiyang Chao, and Dr. Yu Gu for their guidance and support in this effort. Giving me the chance to work throughout the past couple of years in the Flight Control Systems lab has immeasurably changed my future as an engineer.

I would like to thank West Virginia University and in particular the Benjamin M. Statler College of Engineering and Mineral Resources for being home for the last 6 years. I would like to thank Dr. Victor Mucino, the graduate program director, Dr. Jack Prucz, the MAE department chair, and finally Deborah Willis who helped with all of the logistics of being a graduate student.

I would like to thank the member of the FCSL that I had the privilege to work closely with over the past couple of years. This includes Dr. Srikanth Gururajan, Dr. Brad Seanor, Dr. Matt Rhudy, Amanda McGrail, Tanmay Mandal, Caleb Rice, Kyle Lassak, Matteo Guerra, Giovanni De Nunzio, and Matteo Dariol. I would also like to thank the undergraduate students that helped in the lab including Clint Smith, Matthew Underwood, Mathew Milanese, Sean Belardo and Lucas Behrens.

The research conducted during this project could not have been accomplished if it weren't for the extremely talented and proficient pilot's including Mike Eden, Mike Spencer, and Dave Ellis.

I would like to thank my parents who have always been there for guidance and advice and have always given their love and support. I would like to thank my brothers, Andy and Ryan, for their support as well and for always being there.

I would like to thank my wife and kids for supporting me through graduate school over the last couple years. More than anyone my wife has been a constant support and has shown me love beyond what I deserve and thank her for all that she has done.

Finally, I would like to thank my Lord and Savior, Jesus without whom any accomplishments I make would be worth nothing. I owe him my all. 


\section{Table of Contents}

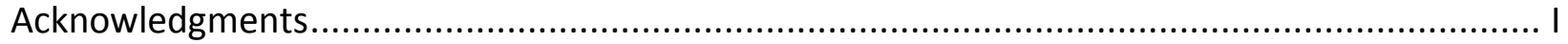

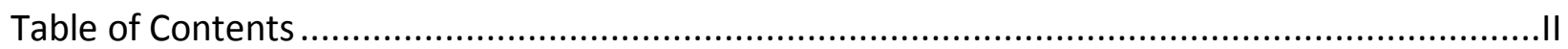

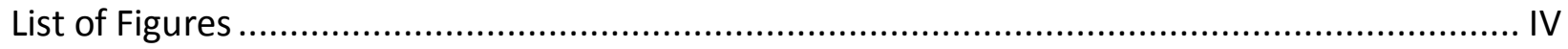

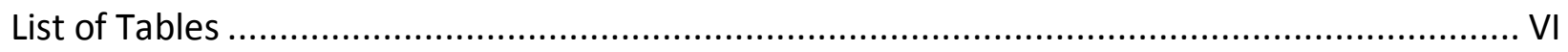

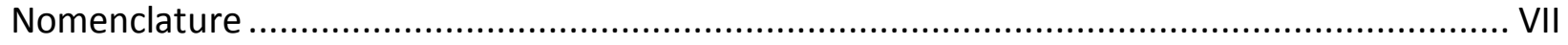

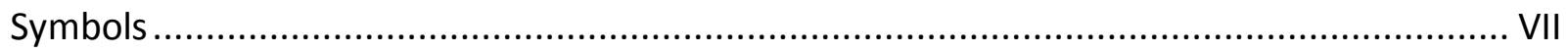

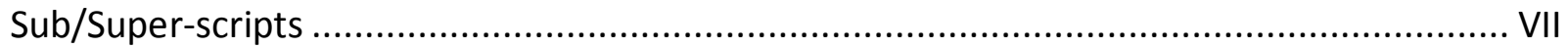

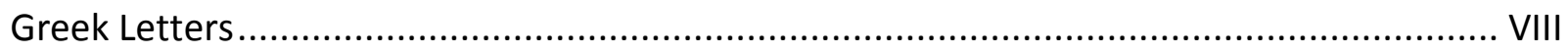

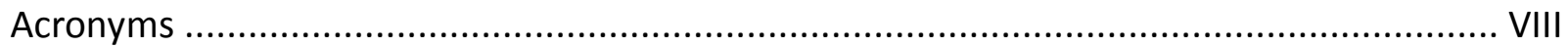

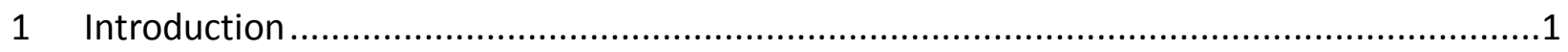

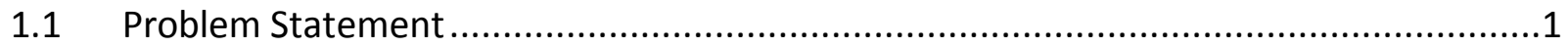

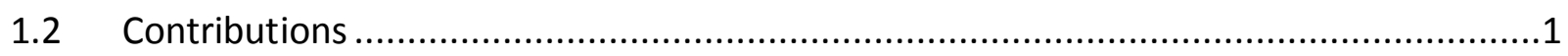

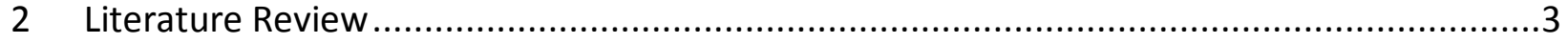

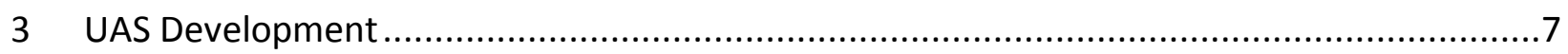

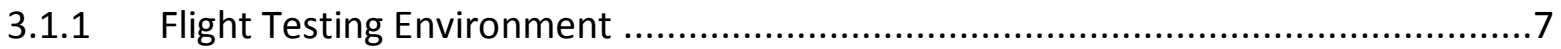

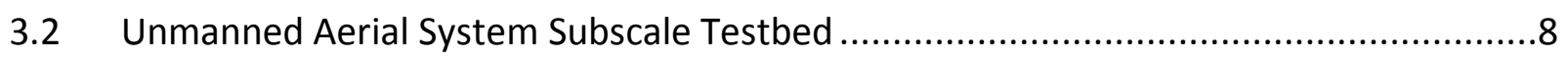

3.2.1 Phastball Research UAV Subscale Testbed ….......................................................8

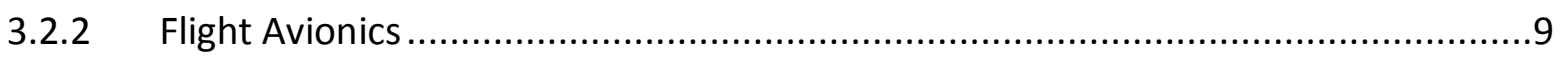

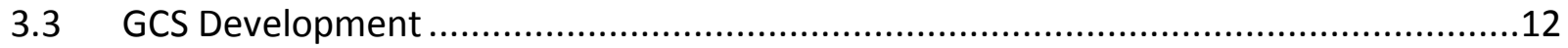

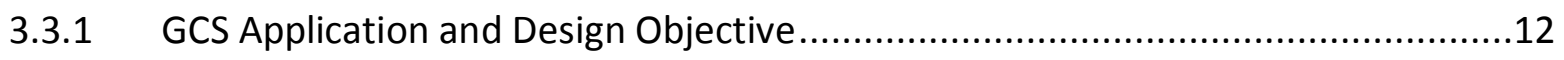

3.3.2 GCS Hardware System..................................................................................13

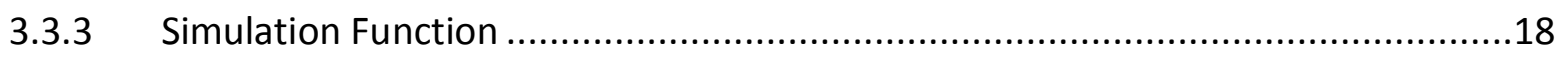

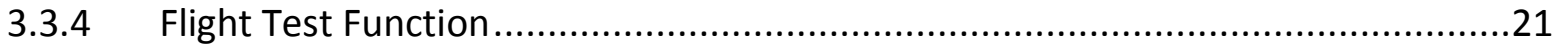

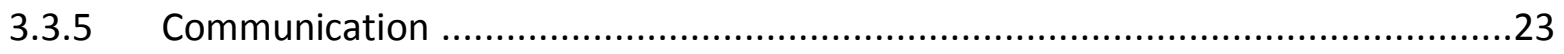

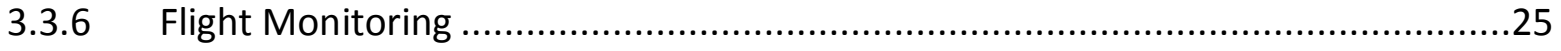

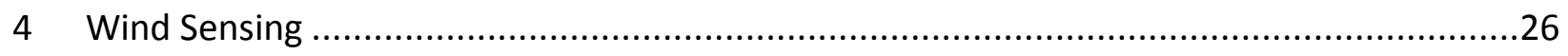

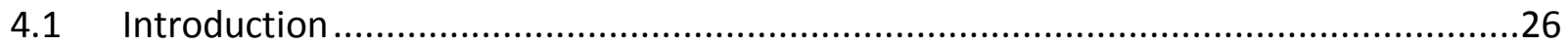

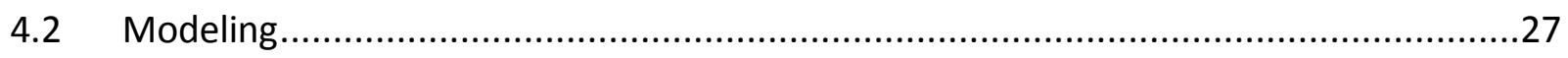

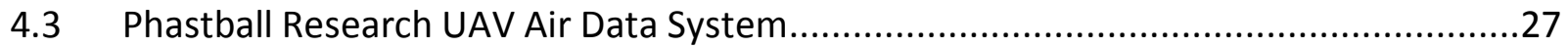




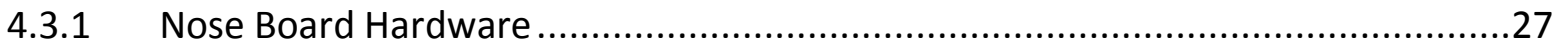

4.3.2 Weather Station for Ground Truth .............................................................30

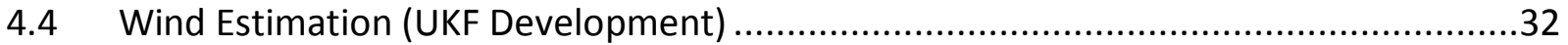

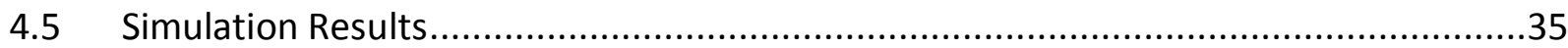

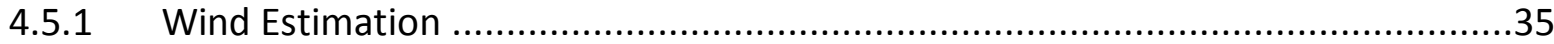

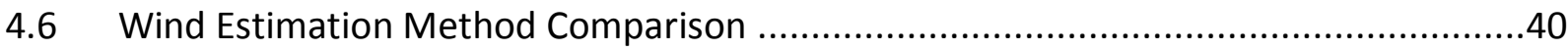

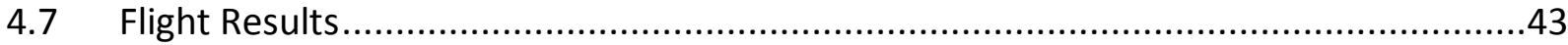

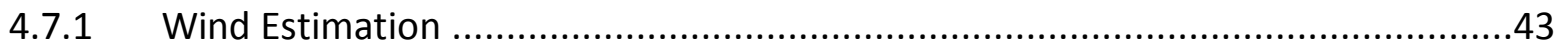

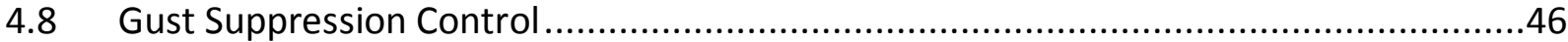

5 Wake Sensing with UAV Formation Flight ..............................................................50

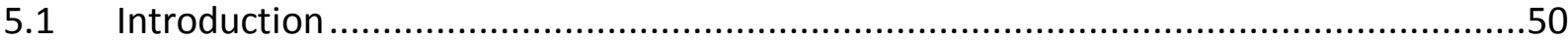

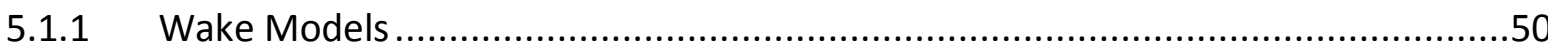

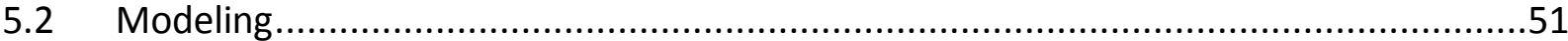

5.3 Phastball Research UAV Formation Flight Setup.....................................................55

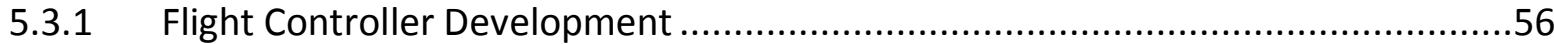

5.4 Wake Sensing Through Flight Tests ....................................................................59

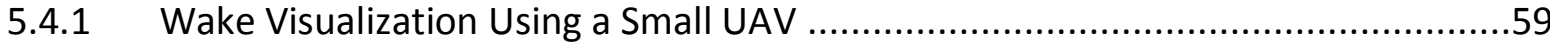

5.4.2 Wake Sensing During Formation Flight .....................................................61

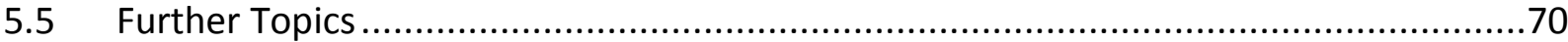

5.5.1 Sweet Spot for Maximum Energy Savings........................................................70

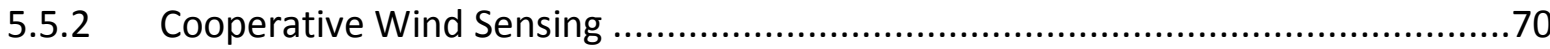

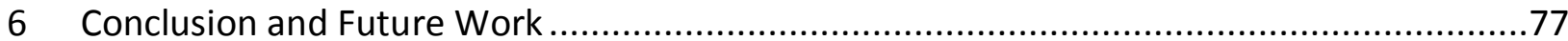

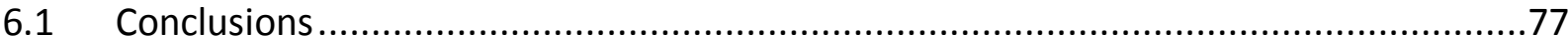

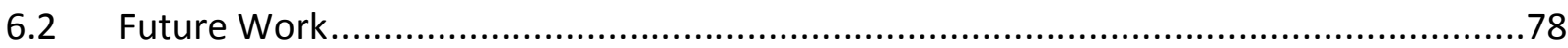

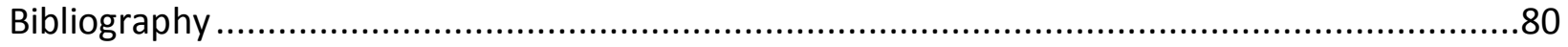




\section{List of Figures}

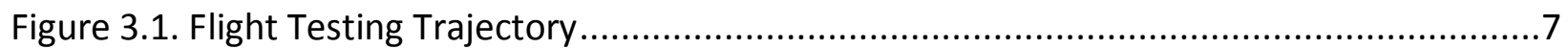

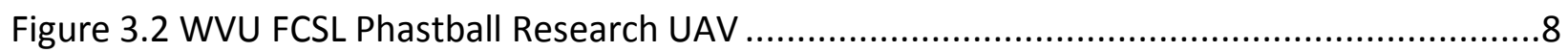

Figure $3.3 \mathrm{Gen}-\mathrm{V}$ avionics system (reproduced from ${ }^{32}$ ) ....................................................10

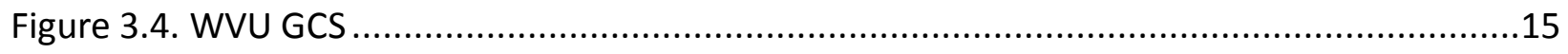

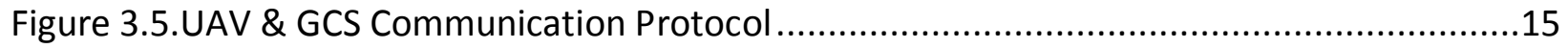

Figure 3.6. Message Tree Showing Partial Downlink Data During Flight ....................................16

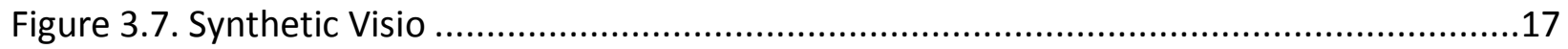

Figure 3.8. GCS display windows: Primary Flight, Overhead Map, and Surfaces Displays ...........17

Figure 3.9. Simulator \& GCS Communication Protocol..........................................................18

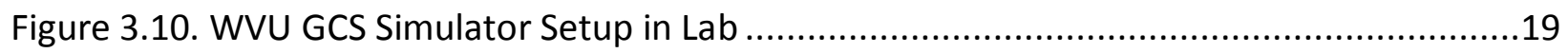

Figure 3.11. Conceptual Representation of Simulator Designed in Simulink ............................20

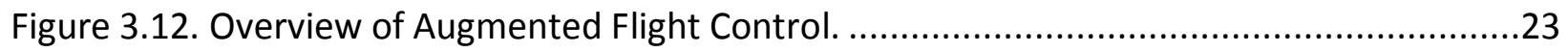

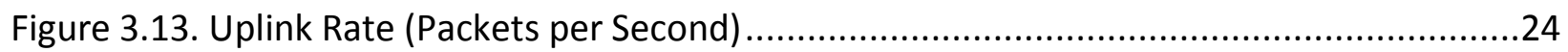

Figure 3.14. Uplink Packets Received During GCS Controller Flight ......................................25

Figure 4.15 One minus cosine wind idealization (reproduced from 40) ....................................27

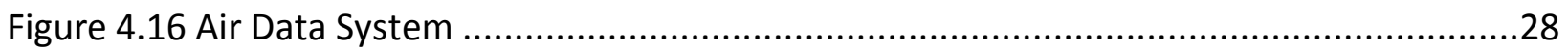

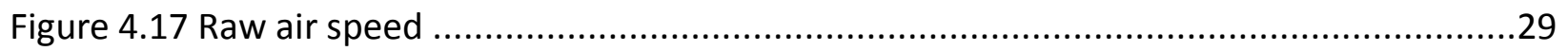

Figure 4.18 Calibrated air speed ........................................................................................

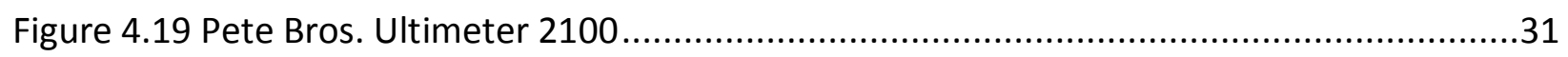

Figure 4.20 Weather station setup during flight testing ..........................................................

Figure 4.21 UKF estimation of wind for a constant wind field ...................................................3

Figure 4.22 UKF estimation of wind for a constant wind field ................................................38

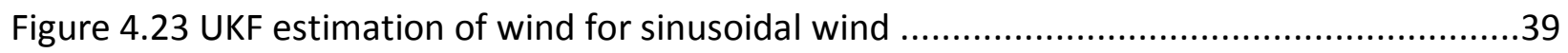

Figure 4.24 Wind triangle for direct method wind calculation ................................................40

Figure 4.25 Wind estimation comparison between UKF and direct calculation ..........................41

Figure 4.26 Wind estimation comparison between UKF and direction calculation .....................42

Figure 4.27 Wind speed at varying altitude (reproduced from NASA Glenn Research Center) ...43

Figure 4.28 UKF wind estimation and weather station data.....................................................45 
Figure 4.29 Induced wind from the leader's wake onto the follower ..................................48

Figure 4.30 Vertical acceleration of the follower UAV as it encounters the leader's wake ........48

Figure 4.31 Vertical acceleration of the follower UAV as it encounters the leader's wake .........49

Figure 5.32 Wake modeling for multiple Phastball Research UAVs in formation flight ..............52

Figure 5.33 Wake model sketch (reproduced from faa.gov) ...........................................52

Figure 5.34 Induced vertical velocity from the wake of the Phastball Research UAV ................55

Figure 5.35 Phastball Research UAV formation flight...................................................56

Figure 5.36 Wake visualization using smoke and small UAV ..........................................60

Figure 5.37 Wake visualization using smoke and small UAV (detailed) .................................61

Figure 5.38 Follower aircraft data during normal flight................................................63

Figure 5.39 Follower flight data during formation flight ............................................63

Figure 5.40 Follower wake sensing data during formation flight .....................................66

Figure 5.41 Follower wake sensing data during formation flight ....................................67

Figure 5.42 Follower sensor data during wake sensing .............................................68

Figure 5.43 Follower wake sensing data during formation flight....................................69

Figure 5.45 Wind field used for UKF wind estimation of leader UAV .................................72

Figure 5.46 Wind field used for UKF wind estimation of follower UAV ..............................72

Figure 5.47 Induced wind on follower from the leader's wake .......................................73

Figure 5.48 UKF wind estimation using flight data from leader only ...................................73

Figure 5.49 UKF wind estimation using flight data from follower only ..................................74

Figure 5.50 UKF wind estimation using cooperative wind estimation techniques ...................74

Figure 5.51 Wind states of UKF estimation using follower data .......................................75

Figure 5.52 Wind states of UKF estimation using cooperative flight data ............................75 


\section{List of Tables}

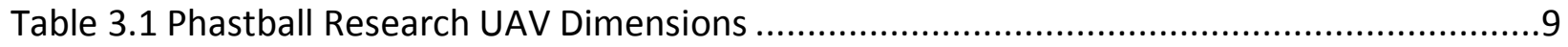

Table 3.2 Phastball Research UAV sensor information.....................................................11

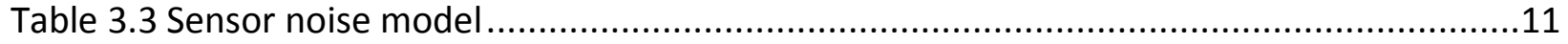

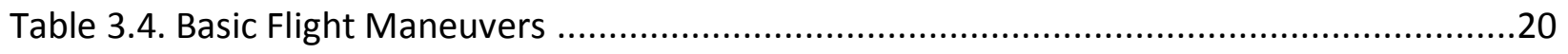

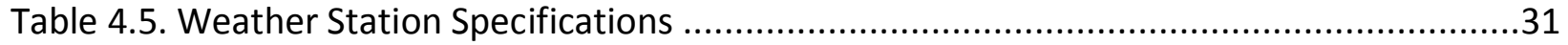

Table 4.6 Update and measurement equations for the UKF .........................................33

Table 4.8 Wind estimation comparison statistics .....................................................42

Table 4.7 Gust suppression control statistics .......................................................... 47

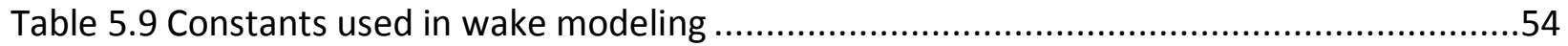

Table 5.10 Variable separation control characteristics ....................................................59

Table 5.11 Cooperative wind estimation statistics .......................................................76 


\section{Nomenclature}

\begin{tabular}{lll|lll} 
Symbols & & & \\
$\widehat{W}$ & wind velocity estimation & $\mathrm{m} / \mathrm{s}$ & $\mathrm{T}$ & temperature & \\
$\mathrm{B}$ & wing span & $\mathrm{m}$ & $\mathrm{u}$ & body axis $\mathrm{o}$ velocity & $\mathrm{m} / \mathrm{s}$ \\
$\mathrm{b}$ & half wing span & $\mathrm{m}$ & $\mathrm{U}$ & output \\
$\mathrm{E}[\cdot]$ & expectation operator & & $\mathrm{V}$ & velocity & $\mathrm{m} / \mathrm{s}$ \\
$\mathrm{g}$ & gravity & $\mathrm{m} / \mathrm{s}^{2}$ & $\mathrm{v}$ & body axis y velocity & $\mathrm{m} / \mathrm{s}$ \\
$\mathrm{m}$ & mass & $\mathrm{kg}$ & $\mathrm{V}$ & induced velocity & $\mathrm{m} / \mathrm{s}$ \\
$\mathrm{p}$ & roll rate & $\mathrm{deg} / \mathrm{s}^{2}$ & $\mathrm{w}$ & body axis z velocity & $\mathrm{m} / \mathrm{s}$ \\
$\mathrm{P}$ & pressure & $\mathrm{Pa}$ & $\mathrm{W}$ & wind velocity & $\mathrm{m} / \mathrm{s}$ \\
$\mathrm{P}$ & position & $\mathrm{m}$ & $\mathrm{x}$ & distance from wake & $\mathrm{m}$ \\
$\mathrm{q}$ & pitch rate & $\mathrm{deg} / \mathrm{s}^{2}$ & $\mathrm{X}$ & generation \\
$\mathrm{r}$ & yaw rate & $\mathrm{deg} / \mathrm{s}^{2}$ & $\mathrm{z}$ & altitude & \\
$\mathrm{R}$ & radius & $\mathrm{m}$ & & & $\mathrm{m}$ \\
$\mathrm{s}$ & wing area & $\mathrm{m}$ & &
\end{tabular}

\section{Sub/Super-scripts}

\begin{tabular}{ll|ll} 
0 & initial & long & longitudinal \\
ail & aileron & $\mathrm{N}$ & North \\
AS & airspeed & Pitot & Pitot tube \\
C & core & rud & rudder \\
corr & corrected & S & static \\
D & Down & tot & total \\
d & differential & uncorr & uncorrected \\
E & East & w & wind \\
elev & elevator & $x$ & x direction \\
F & follower & y & y direction \\
GPS & global positioning system & $\mathrm{z}$ & z direction \\
L & leader & $\alpha$ & wind power law coefficient \\
lat & lateral & $\theta$ & rotational \\
P & power law & &
\end{tabular}




\begin{tabular}{|c|c|c|c|c|c|}
\hline$\alpha$ & angle of attack & deg & $\pi$ & pi & NA \\
\hline$\beta$ & sideslip angle & deg & $\rho$ & air density & $\mathrm{kg} / \mathrm{m}^{3}$ \\
\hline$\Gamma$ & circulation & vorticity*m ${ }^{2}$ & $\tau$ & Turbulence level & N/A \\
\hline$\delta$ & surface deflection & deg & $\phi$ & roll angle & deg \\
\hline$\zeta$ & $\begin{array}{l}\text { gust suppression sign } \\
\text { control }\end{array}$ & $\mathrm{N} / \mathrm{A}$ & $\begin{array}{l}\varphi \\
\psi\end{array}$ & $\begin{array}{l}\text { rotation about wake } \\
\text { yaw angle }\end{array}$ & $\begin{array}{l}\text { deg } \\
\text { deg }\end{array}$ \\
\hline & pitch angle & deg & $\omega$ & vortex descent speed & $\mathrm{m} / \mathrm{s}$ \\
\hline
\end{tabular}

\section{Acronyms}

ADS ...............Air Data System

ARIA ..............Advanced Research Integrated Avionic

EKF................Extended Kalman Filter

FCSL ..............Flight Control Systems Laboratory

FDC ............... Flight Dynamics and Control toolbox

GPS ...............Global Positioning System

ILC................... Inner Loop Controller

IMU................Inertial Measurement Unit

OLC ................ Outer Loop Controller

UAV.................Unmanned Aerial Vehicle

UKF ................Unscented Kalman Filter

WVU ...............West Virginia University

std.................Standard Deviation

NED ................. North East Down

FPV.................. First Person View 


\section{Introduction}

\subsection{Problem Statement}

Generally UAVs have a smaller size and weight and, therefore, are more sensitive to wind and turbulence in the air. Thus, it is important to understand the characteristics of both the wind field that the aircraft is flying through as well as turbulence a UAV may encounter. Another disadvantage of many UAVs is their limited payload and, therefore, a limited endurance; therefore, fuel efficiency and energy harvesting are important issues. Ways to increase endurance through energy harvesting techniques includes both wind soaring, which requires knowledge of the local wind field, and formation flight where a follower aircraft may use the wake of a leader aircraft to increase lift and reduce fuel consumption.

The goal of this research is to develop an accurate and inexpensive system utilizing commonly used to estimate the local wind around an aircraft as well as sense the wake in a leader-follower formation flight. In order to accomplish this task an Unscented Kalman filter (UKF) was developed for estimating the magnitude and direction of the wind which was compared to a ground truth using data from two ground control stations. Next, a flight controller was introduced in order to allow for formation flight and wake sensing using an air data sensor system. Finally, simulations were conducted to show the improvements of using cooperative wind and wake sensing where the previously introduced UKF was modified for the follower aircraft to use the wind estimate of the leader aircraft.

\subsection{Contributions}

The most relevant contributions of this research is a novel way to estimate wind states using a UKF, and Air Data system (ADS), GPS and IMU sensors. Another contribution is that this research also provides some of the first ever wake sensing data of UAVs in formation flight where the follower aircraft is flying completely autonomously. Finally, there are some simulation results showing the potential benefits of cooperative wind and wake sensing along with gust suppression control. 
The rest of this thesis is organized as in the following. Chapter 0 provides a brief literature overview of what has been done in the field of wind and wake sensing as well as development of leading edge GCSs. Chapter 0 describes the development of the UAS used for this research effort including the UAV and its sensor suit used for flight testing as well as the GCS used for aircraft control and simulation. Chapter 4 discusses how wind sensing was accomplished through both simulation and flight test including modeling, UKF development, and fight data. Chapter 0 shows the modeling used for the leader's wake, wake visualization using a smoke screen, and flight data showing the follower sensing the leader's wake. This chapter also shows simulation results for cooperative wind and wake sensing and gust suppression control. Finally, Chapter 0 concludes the document with recommendations for this research project as well as future work of this research. 


\section{Literature Review}

Wind and turbulence have a large impact on aircraft flight performance, energy consumption, and aviation safety, especially for small and micro Unmanned Aerial Vehicles (UAVs). In nature, albatrosses are able to travel thousands of miles by soaring dynamically through the wind gradient generated by ocean waves. With the aid of thermal winds, large birds such as hawks can stay aloft for hours without a single wing beat ${ }^{1}$. Birds are also known to use atmospheric turbulence such as from the wake of another bird in order to increase its flight range. Many species of birds are known to be able to boost their range and efficiency through formation flight, where the following bird will ride the wake induced by the leading bird $^{2}$. It has been shown that a flock of 25 birds could increase their range by up to 70 percent that an individual bird could achieve on its own ${ }^{3}$. Similarly, the use of wind information during navigation, such as thermal soaring and formation flight, can significantly increase the flight endurance of small $\mathrm{UAVs}^{4,5,6}$. It is envisioned that in the near future small UAVs will be increasingly deployed in dangerous situations such as tornado chasing, forest fires, chemical leaks, nuclear contamination, and for meteorological measurement and aerial observations ${ }^{7}$.

UAVs with customized instrumentation can offer relatively inexpensive airborne research platforms for providing real-time wind-field measurements with high spatial and temporal resolutions. For the purpose of wind estimation, the three-vector method was proposed in 1998 for manned aircraft to determine the wind speed through a combination of three known ground velocity vectors to find the circle center with the assumption of constant airspeed and wind speed ${ }^{8}$. A similar approach is the "two-vector method" which uses two ground velocity and heading measurements to form two triangles for wind speed and airspeed estimation ${ }^{9}$. In the last two decades, stochastic filters are increasingly used for the wind estimation problem on UAVs, including Extended Kalman filters (EKF) $)^{10,11}$ and Unscented Kalman filters (UKF) ${ }^{12}$. A simple EKF was proposed for the 2D constant wind estimation using only the GPS and Pitottube sensor ${ }^{10}$. Other EKFs were developed based on a more complete set of airborne sensor suites available to small UAVs [10]. Other than the wind triangle relationship, aircraft dynamic

response can also be utilized for wind estimation ${ }^{13,14}$. It is worth mentioning that most of the above approaches did not require the measurements from air flow angle sensors, which makes 
it difficult to estimate the 3D wind. However, such capabilities are crucial to the wake sensing problem because there are significant vertical components from wake vortices and for. The knowledge of vertical wind components is also critical for energy harvesting through thermal soaring or flying with the sweep spot ${ }^{16}$ of the leader's wake.

The majority of wake modeling research has been conducted on manned aircraft to help understanding and mitigating the potential dangers of an aircraft encountering another aircraft's wake. Also, by understanding the characteristics of wakes produced by aircraft, it becomes possible to increase air traffic while maintaining safety from unwanted wake encounters. In recent years, wake modeling has been focused using small UAVs ${ }^{15}$. These approaches often require substantial on-board computing power to calculate the wake in real time; additionally, they often require pre-processing data to fully understand the wake characteristics.

Although the general shape and position of trailing vortices have been extensively studied ${ }^{16}$, there is still a big gap between simulated results and real UAV flight tests. Real-time wake sensing will allow aircraft to fly in close formation and benefit from the trailing vortices of the leader's aircraft by maintaining a position within the upwash, or sweet spot of the leader's $\operatorname{vortex}^{17}, 18$. Another reason for using UAVs for wake sensing for energy harvesting is that it has been shown in reent flight testing programs that it well beyond the pilots ability to maintain control inside of the wake for extended periods of time ${ }^{19,18}$. The high workload associated with flying in the sweet spot of the leader's wake is due to the face that the pilot cannot trim the aircraft because of the random nature of the turbulence within the wake. It is also important to point out that simulations have shown that there are regions within the wake where an aircraft may not have the control authority to successfully trim ${ }^{16}$.

One of the major benefits of being able to sense the surrounding wind and the wake of another aircraft is to increase the endurance and, therefore, range of the aircraft. UAVs that have a flight range beyond the range of sight of the pilot need some type of external display for the pilot to see what the UAV is doing. In smaller applications this can accomplished through First Person View (FPV) flight where the pilot looks at a screen that usually displays flight data 
and a flight video from the UAV. On larger applications it is necessary for the pilot to fly from a replicated cockpit in order to have access to useful flight data, video, and controls and therefore, they are often flown through the use of a Ground Control Station (GCS). It is becoming increasingly common for military and scientific research to require the development of customized UAV GCSs for their specific objectives. Therefore, different types of UAV GCSs have been ad-hoc designed and developed. The military relies heavily on the capabilities of GCSs for control and mission planning of UAVs such as the Reaper or the Predator in various missions including surveillance, reconnaissance, and strike tasks ${ }^{20}$, which are usually more tolerant to system delays, that could be several seconds. Similarly, the ANKA program designed by the Turkish Aerospace Industries and used by the Turkish Air Force has developed a GCS for control and monitoring of an air vehicle in real-time as well as mission planning, data recording, and querying that is non-real-time ${ }^{21}$.

Not only military but several universities, research centers, and private companies have developed GCSs for research with various types of UAVs such as Berkeley's BEAR research facility $^{22}$ used mainly for rotorcraft while the previously mentioned AirSTAR is used for research into aviation safety by testing loss of control scenarios without risk to a piloted aircraft ${ }^{23}$. L-3 Unmanned Systems has developed a portable command and control station for UAVs that is available commercially ${ }^{24}$. However, most of the GCS configurations are designed either for simple monitoring functions, or for expensive military or governmental missions, which are not suitable for universities or small companies. It is envisioned that low-cost, compact yet capable UAV GCS solutions are needed by researches specifically involved in pilot modeling, pilot training, etc.

As an interface between human pilot and the unmanned system, UAV GCSs can also be used for Human Systems Integration (HSI) research with the general goal of enhancing the capabilities of the overall pilot-UAV system. A group from the Air Force Research Laboratory along with Arizona State University Polytechnic has researched the pilot's ability for navigation and orientation within the virtual environment, the modeling of the pilot's cognitive process within maneuvering and reconnaissance missions, verbal communication between pilot and 
synthetic entities, as well as analysis of team skill ${ }^{25}$. Another research effort has focused on how to reduce the workload of UAV operators while at the same time mitigating safety issues when the number of aircraft being controlled increases compared to the number of operators ${ }^{26}$. Not only has this been done using single modes of communication between pilots and GCSs, but efforts have gone into how to combine these into multimodal approaches for reducing load by increasing the avenues from which the operator may receive information ${ }^{27}$. Another piece of valuable information is determining what information the pilot is most interested in for UAV control purposes. Through eye tracking of the pilot, research has shown what data the pilot is most concerned about ${ }^{28}$. Along similar lines of research, the University of North Dakota has developed a mobile information display, similar to a GCS without control, that is designed specifically for detecting low flying, observable aircraft ${ }^{29}$. These research topics have shown a great interest in understanding not only how the pilot controls the UAV through the GCS, but also how to best improve the interaction between the pilot and the GCS. 


\section{UAS Development}

The following section describes the experimental test bed used for simulations and flight testing. For modeling and theory validation it is important to have accurate data that captures the research objective. This section discusses the UAV used for flight testing and collecting data as well as the GCS developed to augment the system.

\subsubsection{Flight Testing Environment}

Flight testing was conducted at the Louis Bennet Field. This facility, located in Jackson Mill (WV), is owned by West Virginia University. The air field consists of a paved runway approximately 950 meters long and 15 meters wide and running 10deg off North to the west. Flights are usually conducted between April and December and consist of flying a racetrack type loop around the runway. From takeoff to landing each flight is approx. 6 minutes in length of which 5 minutes are used for research purposes. Figure 3.1 shows a typical flight path overlaid on a Google ${ }^{\circledR}$ map of the runway in Jane Lew, West Virginia.

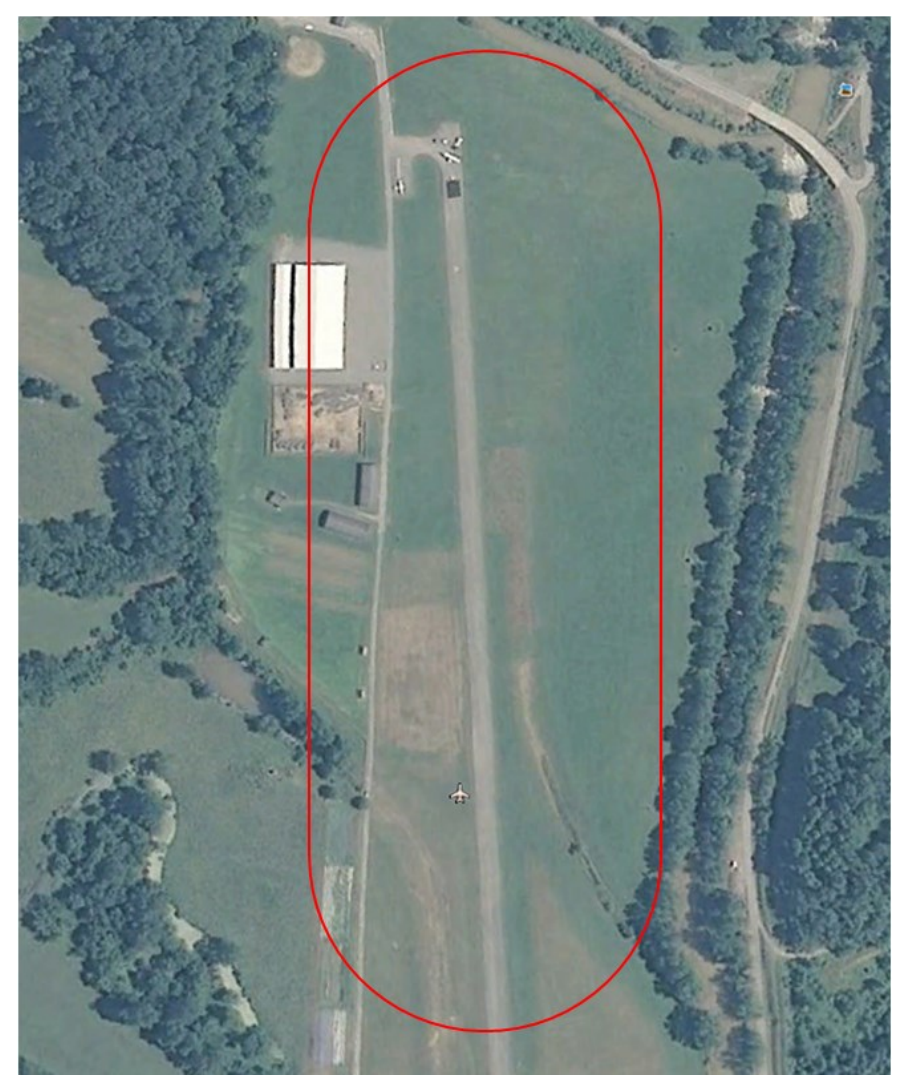

Figure 3.1. Flight Testing Trajectory 


\subsection{Unmanned Aerial System Subscale Testbed}

\subsubsection{Phastball Research UAV Subscale Testbed}

The WVU Phastball research UAV research aircraft is an unmanned aircraft designed, manufactured, and instrumented by researchers at the WVU FCSL ${ }^{[30]}$ in the Mechanical and Aerospace Engineering Department The FCSL currently has two Phastball research UAVs featuring the same set of sensors and instrumented with the Advanced Research Integrated Avionics (ARIA) system (later described in in Section 3.1.2).

The Phastball research UAV is a mid-wing, T-tail aircraft with a tricycle landing gear; it is propelled by two ducted fans mounted just aft of the wing, as shown in Figure 3.2. The wing is separated into two sections. The mid-section features a NACA 2410 airfoil; the two duplicate tip sections are tapered and are capped with a winglet. Both the horizontal tail and vertical tail feature a NACA 0009 airfoil. Control surfaces on the Phastball research UAV include two ailerons, two elevator, and one rudder; each surface is controlled by a digital servo.

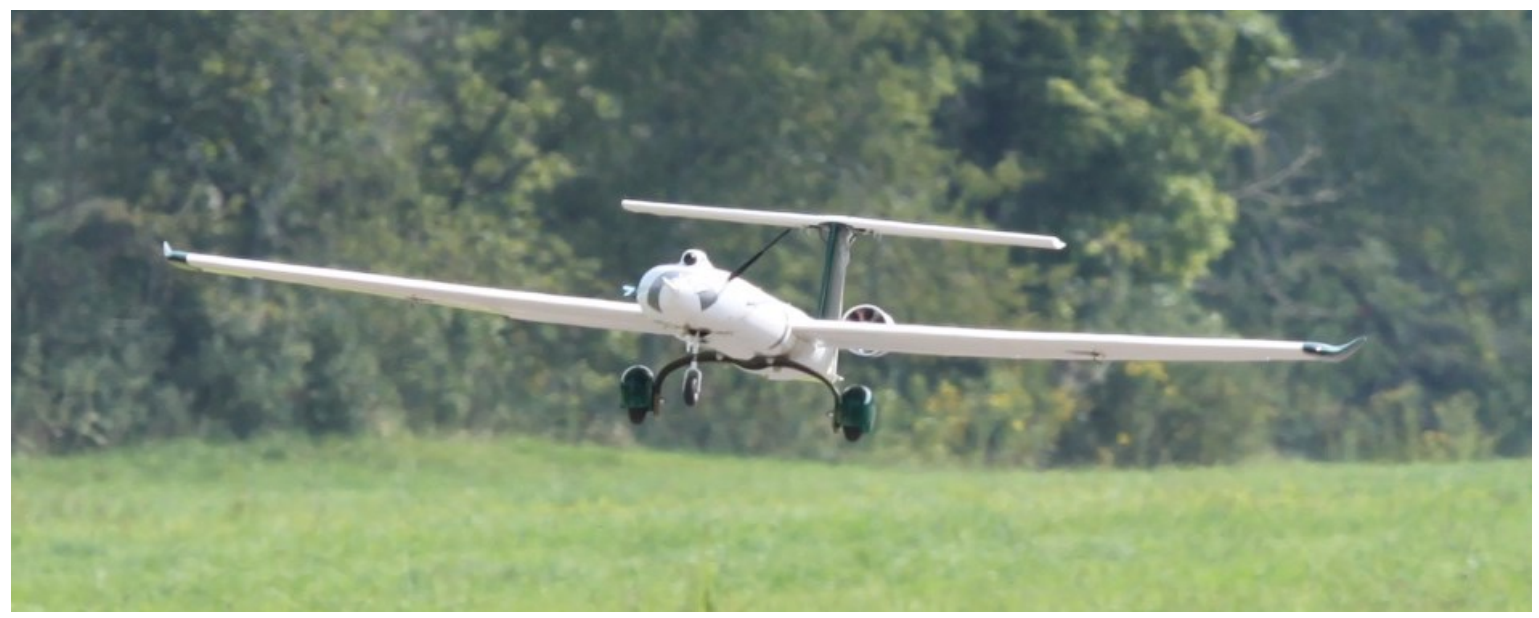

Figure 3.2 WVU FCSL Phastball Research UAV

The fuselage of the Phastball research UAV is manufactured with carbon fiber and fiber glass composite with plywood bulkheads. The wing contains a foam core with carbon rod spar running the length of the wing. The Phastball research UAV is powered by two ARC 36-55-1.5 electric brushless motors with Skyworld $90 \mathrm{~mm}$ mounted ducted fans. The takeoff weight is 
approximately 24 pounds. Detailed geometric characteristics and specifications for the Phasball are shown below in Table 3.1.

Table 3.1 Phastball Research UAV Dimensions

\begin{tabular}{|c|c|c|c|c|c|c|}
\hline & Parameter & Specs & & & Parameter & Specs \\
\hline \multirow{4}{*}{$\frac{\frac{\pi}{\pi}}{\frac{\pi}{2}}$} & Length & $2.2 \mathrm{~m}$ & \multirow{4}{*}{\multicolumn{2}{|c|}{ 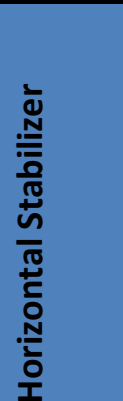 }} & Span & 0.86 \\
\hline & Height & $\begin{array}{l}0.27 \mathrm{~m} \text { at nose } \\
0.55 \mathrm{~m} \text { at tail }\end{array}$ & & & Airfoil & NACA 0009 \\
\hline & GTOW & $11 \mathrm{~kg}$ & & & $\begin{array}{l}\text { Mean Aerodynamic } \\
\text { Chord }\end{array}$ & $0.21 \mathrm{~m}$ \\
\hline & Cruise Speed & $30 \mathrm{~m} / \mathrm{s}$ & & & Planform Area & $0.18 \mathrm{~m}^{2}$ \\
\hline \multirow{4}{*}{$\stackrel{\infty}{\stackrel{\infty}{\lessgtr}}$} & Wingspan & $2.23 \mathrm{~m}$ & \multirow{4}{*}{ : } & \multirow{4}{*}{ 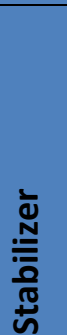 } & Span & $0.28 \mathrm{~m}$ \\
\hline & Airfoil & NACA 2410 & & & Airfoil & NACA 0009 \\
\hline & $\begin{array}{l}\text { Mean Aerodynamic } \\
\text { Chord }\end{array}$ & $0.33 \mathrm{~m}$ & & & $\begin{array}{l}\text { Mean Aerodynamic } \\
\text { Chord }\end{array}$ & $0.29 \mathrm{~m}$ \\
\hline & Planform Area & $0.73 \mathrm{~m}^{2}$ & & & Planform Area & $0.16 \mathrm{~m}^{2}$ \\
\hline
\end{tabular}

\subsubsection{Flight Avionics}

\subsubsection{Computer and Operating System}

The Phastball research UAV features a computer system, called "ARIA" (Advanced Research Integrated Avionics), designed at the FCSL at WVU for fault tolerant research ${ }^{31}$. ARIA, shown in Figure 3.3, contains four stacked printed circuit boards (PCB). ARIA is the $5^{\text {th }}$ generation of avionics systems developed for the UAVs at the FCSL. The bottom board houses an IMU and acts as the main sensor interface, and the signal distribution controller. A Netburner MOD 5213 microprocessor is used for low-level control including interfacing with the IMU, receiving pilot commands, and generating surface and throttle PWM commands. The central processor is a Diamond System's Athena II general purpose Single Board Computer containing 16 channels of 16 bit data acquisition and four serial ports. 
The operating system is a Suse Linux with a Real Time Application Interface (RTAI) patch ${ }^{31,32}$ which runs from a bootable compact flash card. This patch enables flight schemes to be developed within Matlab ${ }^{\circledR}$ and Simulink ${ }^{\circledast}$ which can be converted into C files using Matlab ${ }^{\circledR \prime} \mathrm{S}$ Real Time Workshop, and be compiled into a real-time executable under Linux.

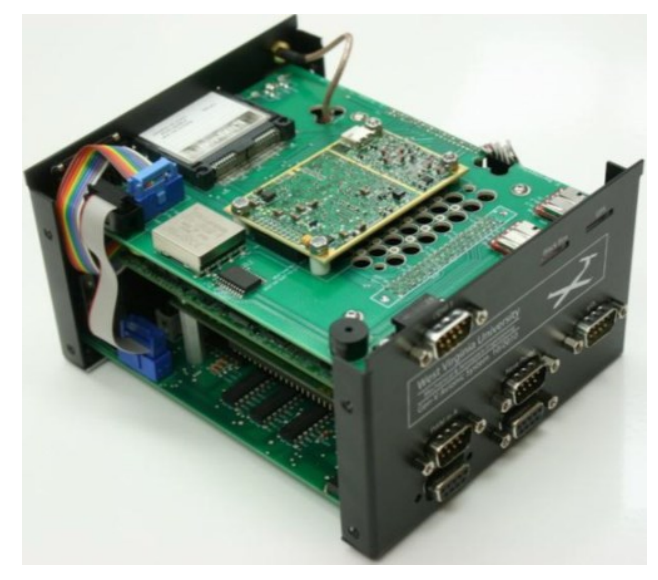

Figure 3.3 Gen-V avionics system (reproduced from ${ }^{31}$ )

\subsubsection{Sensors}

The Phasball platform contains a sensor suit designed to measure all pertinent flight data used both for UAV flight control and research conducted at the FCSL. This suit includes the IMU mounted on the bottom board of the ARIA computer which contains a three axis accelerometer, a three axis gyroscope, and a three axis magnetometer. The UAV also features a GPS receiver for position and velocity measurements, a Pitot tube to measure airspeed, three potentiometers to measure angle of attack and sideslip angle, a laser range finder and a temperature/humidity sensor. Table 3.2 below shows the sensor and their specs.

Previous work at the WVU FCSL consisted of developing a noise model for the sensors used onboard the Phastball research UAV ${ }^{33}$. This sensor model was developed for use in sensor fusion research including the flight evaluation of different stochastic filters for the attitude estimation of aircraft. 
Table 3.2 Phastball Research UAV sensor information

\begin{tabular}{|c|c|c|c|c|}
\hline Sensor & Model & Specs & Measurement & Location \\
\hline GPS & NovAtel OEM-V1 & - & $\begin{array}{l}\text { ECEF Position } \\
\text { and Velocity }\end{array}$ & $\begin{array}{c}\text { Antenna: top } \\
\text { of fuselage }\end{array}$ \\
\hline \multirow{2}{*}{$\begin{array}{l}\text { Accelerometer } \\
\text { Gyroscope }\end{array}$} & ADIS 16355 IMU & $\pm 18 \mathrm{~g}$ & Body Accel & Inside IMU \\
\hline & ADIS 16355 IMU & $\pm 150^{\circ} / \mathrm{sec}$ & $\begin{array}{c}\text { Body Rotational } \\
\text { Rate }\end{array}$ & Inside IMU \\
\hline \multirow{2}{*}{$\begin{array}{l}\text { Magnetometer } \\
\text { Pitot Tube }\end{array}$} & ADIS 16355 IMU & \pm 2.5 gauss & Magnetic Field & Inside IMU \\
\hline & $\begin{array}{c}\text { HCE0811ARH8P5 } \\
\text { (static) } \\
\text { HCEM020DU } \\
\text { (dynamic) }\end{array}$ & $\begin{array}{c}800: 1100 \text { mbar } \\
3 \text { mbar accuracy } \\
0: 20 \text { mbar } \\
0.1 \text { mbar } \\
\text { accuracy }\end{array}$ & $\begin{array}{l}\text { Dynamic and } \\
\text { Static Pressure } \\
\text { used for } \\
\text { Airspeed }\end{array}$ & Nose \\
\hline \multirow{2}{*}{$\begin{array}{l}\text { Potentiometer } \\
\text { Laser Range } \\
\text { Finder }\end{array}$} & MP1545A-S10V & - & Flow Angles & Nose \\
\hline & Opti-logic 261539 & Precision: $\pm 1 \mathrm{~m}$ & Altitude & Nose \\
\hline $\begin{array}{l}\text { Humidity and } \\
\text { Temperature } \\
\text { Sensor }\end{array}$ & HTM2500LF & $\pm 3 \% \mathrm{RH}$ & $\begin{array}{l}\text { Humidity and } \\
\text { Temperature }\end{array}$ & Nose \\
\hline
\end{tabular}

Table 3.3 Sensor noise model

\begin{tabular}{lll}
\hline Sensor Data & Standard Deviation & Units \\
\hline GPS Velocity & 0.03 & $\mathrm{~m} / \mathrm{s}$ \\
GPS Position & 1 & $\mathrm{M}$ \\
Accelerometer & 0.0034 & $\mathrm{G}$ \\
Gyroscope & 0.333 & $\mathrm{deg} / \mathrm{s}$ \\
Potentiometer & 0.025 & $\mathrm{Deg}$ \\
Airspeed & 0.5 & $\mathrm{~m} / \mathrm{s}$ \\
\hline
\end{tabular}




\subsection{GCS Development}

One of the draw-backs of flying UAVs with a transmitter is that there is a large disconnect between flying from the ground and looking at a UAV and actually being in or having a view from the cockpit. As the use of UAVs tends to increase it can be visualized that ground control stations (GCS) will be used to control UAVs flying at great distances, and/or to control multiple UAVs simultaneously, and/or to maintain a human interface for autonomous flight tasks, and so on. Additionally, the GCS is also a useful tool for modeling pilots and training them in the lab. With the setup of a GCS a pilot can be trained through the use of simulators which are close to actual flight. Also, GCS controlled flights mimic manned aircraft flight much more accurately which is useful for modeling and research into manned aircraft using UAVs.

The previous section discussed the UAV test bed used for research and data collection; this section, instead, will focus on the GCS which is used to control the UAVs from the ground. These two elements of the UAS communicate via wireless serial communication along with a safety pilot that remains in visual contact with the UAV to take over should any problem arises. The GCS has two main components, a mobile unit that in the field used to fly the UAVs, and a simulator in the lab used for training and integration of new hardware/software updates.

\subsubsection{GCS Application and Design Objective}

A fully functional GCS provides for research capabilities which cannot be achieved through R/C flight alone. Clearly, the GCS allows pilot modeling with the use of subscale aircraft. Similarly, the use of the GCS is also important for Parameter Identification (PID) research since the pilot no longer has to rely on flying the UAV by visual contact from the ground, but can track actual values of flight from displayed data which helps in performing precise maneuvers used for aircraft modeling. Finally, the use of the GCS naturally leads to research into new applications of subscale aircraft for otherwise dangerous or long mundane tasks ${ }^{34}$. In general the use of a GCS allows for:

1. Pilot modeling at various conditions including Pilot Induced Oscillations (PIO), Loss of Control (LOC) events (which accounted for $59 \%$ of commercial aviation fatalities from 1997-2006 ${ }^{35}$ ), and flights outside of the nominal flight envelope.

2. PID at extreme flight conditions, including high angle of attack, stall conditions, high 
angular velocity, and other.

3. Simulated flight in commercial or military aircraft outside of nominal flight conditions and extreme maneuvers through the use of UAVs.

4. Surveillance using UAVs for situations that require human intelligence and interaction.

5. Communications relay through long duration UAVs that can act as a network in the sky.

The WVU GCS was designed to feature the following capabilities:

1. A ground simulator, to be used for the pilot modeling as well as for pilot training.

2. Flight testing capabilities for verification of pilot and aircraft modeling as well as better pilot-GCS interfacing.

3. Different levels of autonomy for UAVs to support further researches on human systems integration. This includes a manual mode that gives the pilot full control over all control surfaces and throttle settings, an autopilot flight mode that allows for GPS waypoint tracking, and finally, an augmented flight mode that allows for assisted aircraft flight.

In addition, the WVU GCS can also be configured to work with additional R/C vehicles such as unmanned ground/water vehicles.

\subsubsection{GCS Hardware System}

The GCS is comprised of four major components:

1. The Pilot Control Input unit

2. The Communication unit

3. The GCS displaying unit

4. The Central Computer

A rugged computer powered by Intel Core i5-2300 processor serves as the central computing/communications/interface hub. This computer is used for converting pilot inputs to commands and sending them to the wireless modem. It also receives and displays data from the UAV. During the flight testing operations there are two pilots, the safety pilot and the GCS research pilot. The research pilot controls the UAV through pilot control input unit, following Visual Flight Rules (VFR). The safety pilot controls the UAV through RC link and will only take control from the research pilot should the research pilot lose control via a switch on the R/C 
transmitter. The safety pilot is responsible for the take-off and the landing. Figure 3.4 shows the WVU GCS and Figure 3.5 shows the hardware communication protocol. The functions of each subsystem are described below.

\subsubsection{Pilot Control Input Unit}

A Hotas Warthog side stick, CH PRODUCTS Pro pedals, and a Thrustmaster throttle are used for pilot inputs. These devices are used for the generation of the pilot commands for ailerons, elevators, rudder, and throttle as well as for surface trimming and choosing which screens are displayed in the GCS displaying unit (discussed further in Section III.A.3). Each input has a range of 65536 points, or 16 bit resolution. The GCS software converts the raw inputs into an 8 bit commands with a range of 0 to 255 and then sends them out to the onboard flight computer for surface control through wireless communications.

\subsubsection{Communication Unit}

The communication between the GCS and the UAV is based on a $900 \mathrm{MHz}$ serial data link via a pair of FreeWave modems. The GCS modem is connected to the central computer through a serial RS232 connection. The modem antennas used for the GCS and UAV are both omnidirectional antennas with a gain of $5 \mathrm{dBi}$ and $0 \mathrm{dBi}$ respectively. $\mathrm{A} 0 \mathrm{dBi}$ antenna was selected for the UAV because of its smaller size and weight and also because of the changing orientation and position of the UAV during flight tests. Figure 3.5 shows the communication

protocol for GCS hardware. Each uplink packet consists of control commands sent from the pilot station, a 2 byte header, a checksum, and is a total of 16 bytes of data. Downlink packets are 64 bytes in length and include the following: header, checksum, IMU data, GPS data, control commands, air pressure data, flow angles, and attitude angles. 


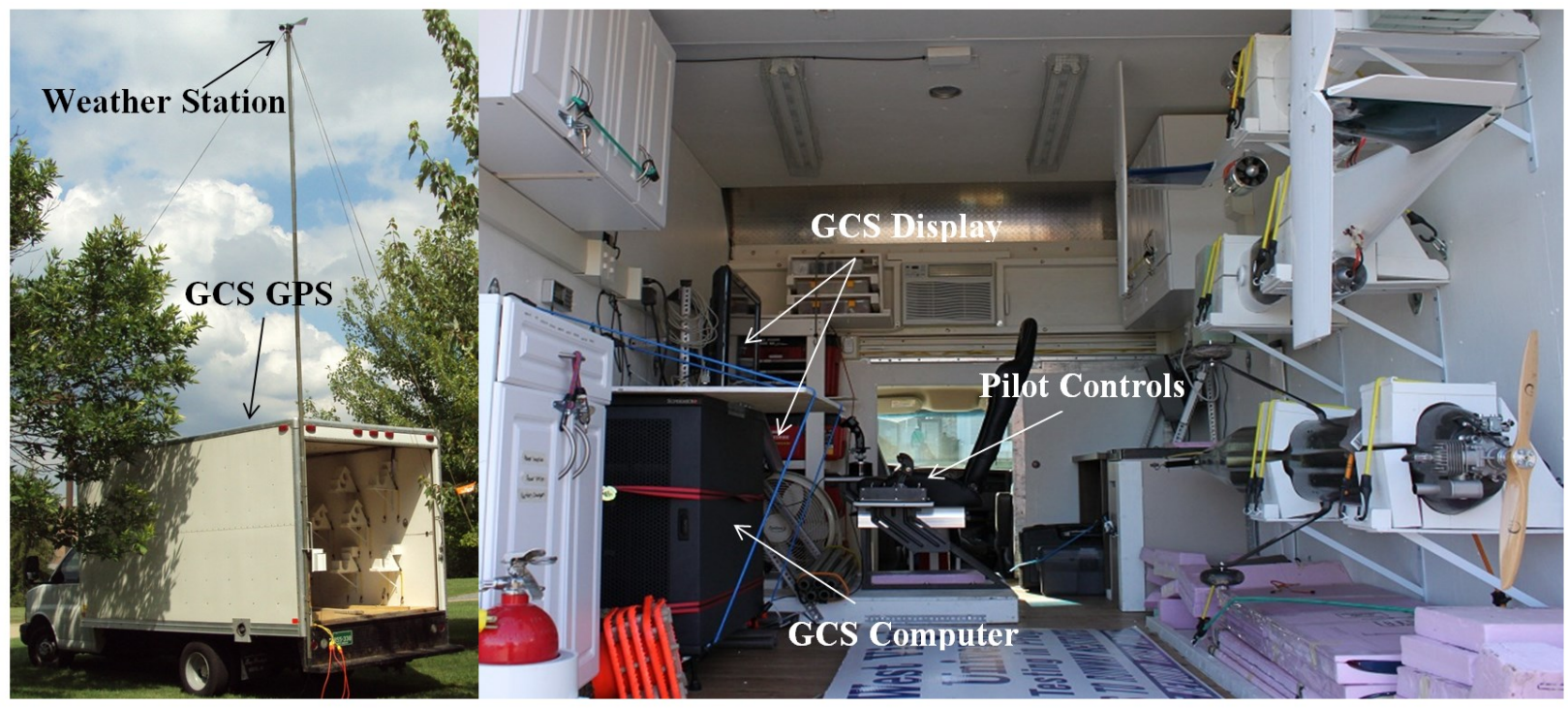

Figure 3.4. WVU GCS

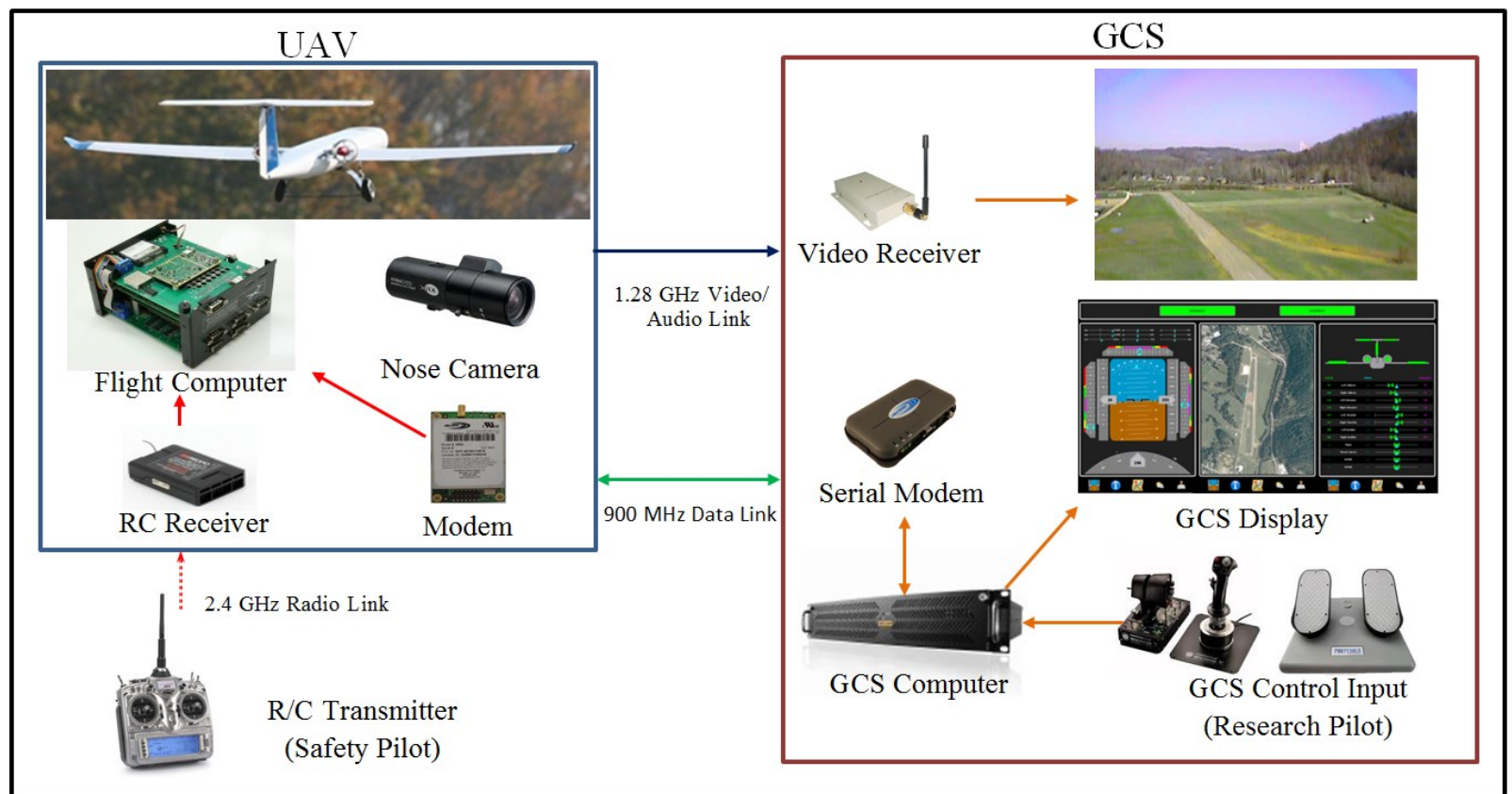

Figure 3.5.UAV \& GCS Communication Protocol

\subsubsection{GCS Display Unit}

The purpose of the GCS display unit is to provide both uplink and downlink data to the research pilot. First, a message tree, shown in Figure 3.6, is used to display text data for each specified parameter. Next, data is displayed in a Heads-Up Display (HUD) overlaid on X-plane for a synthetic vision of flight, as shown in Figure 3.7. Finally, there is a pilot station that can 
display two windows out of a possible of five to visualize the same data in the message tree. A box at the top of the pilot station will display "SAFETY" when the safety pilot is in control of the UAV and will highlight green and display "RESEARCH" when the research pilot is in control of the UAV. The three windows shown in Figure 3.8 are:

1. Primary Flight Display (left): This window contains a virtual horizon that displays pitch and roll angles of the UAV as well as heading. Velocity, altitude, angle of attack, sideslip angle and climb rate are shown on the HUD. Finally, magnetometer and accelerometer data are shown at the top along with angular rates.

2. Overhead Map Display (middle): This window shows a ground track for the UAV as well as the position of the GCS.

3. Surfaces Display (right): This window displays the surface deflections as well as throttle settings. The data is displayed both graphically and numerically for each channel.

\begin{tabular}{|c|c|c|}
\hline \multicolumn{3}{|l|}{$\Delta$ downlink [30928] } \\
\hline count & 208 & Packet Count \\
\hline ps & 46.08 & Static Pressure \\
\hline qc & 321.27 & Dynamic Pressure \\
\hline alpha & -2.78001 & Alpha \\
\hline beta & 0.259993 & Beta \\
\hline airtemp & -273.15 & Air Temperature \\
\hline $\mathrm{p}$ & 0.0599927 & $\mathrm{P}$ \\
\hline$q$ & 0.0199927 & Q \\
\hline r & $-7.30902 \mathrm{e}-06$ & $\mathrm{R}$ \\
\hline$a x$ & 0.0293229 & Acceleration $\mathrm{X}$ \\
\hline ay & 0.0191189 & Acceleration $\mathrm{Y}$ \\
\hline $\mathrm{az}$ & -0.933935 & Acceleration Z \\
\hline $\mathrm{mx}$ & 51650 & Magnetic X \\
\hline my & 21350 & Magnetic $Y$ \\
\hline$m z$ & 233.42 & Magnetic Z \\
\hline pitch & 1.72843 & Pitch \\
\hline roll & 2.30139 & Roll \\
\hline pwm_lele & 134 & Deflection - Left Elevator \\
\hline pwm_rele & 123 & Deflection - Right Elevator \\
\hline pwm_lail & 91 & Deflection - Left Aileron \\
\hline pwm_rail & 104 & Deflection - Right Aileron \\
\hline
\end{tabular}

Figure 3.6. Message Tree Showing Partial Downlink Data During Flight 


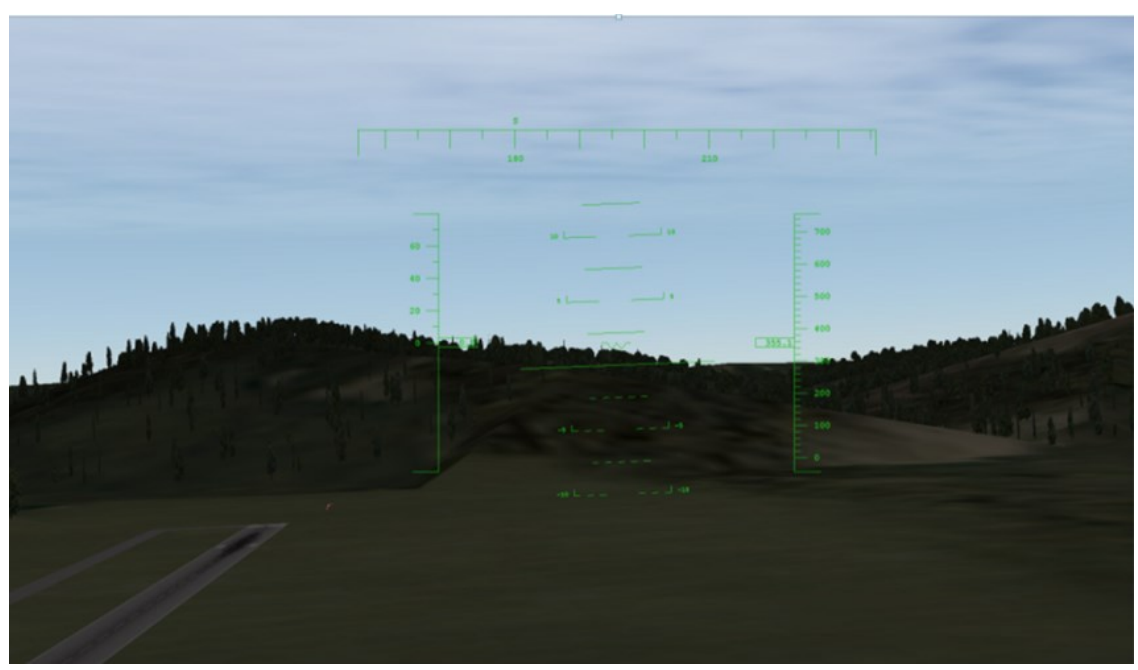

Figure 3.7. Synthetic Visio

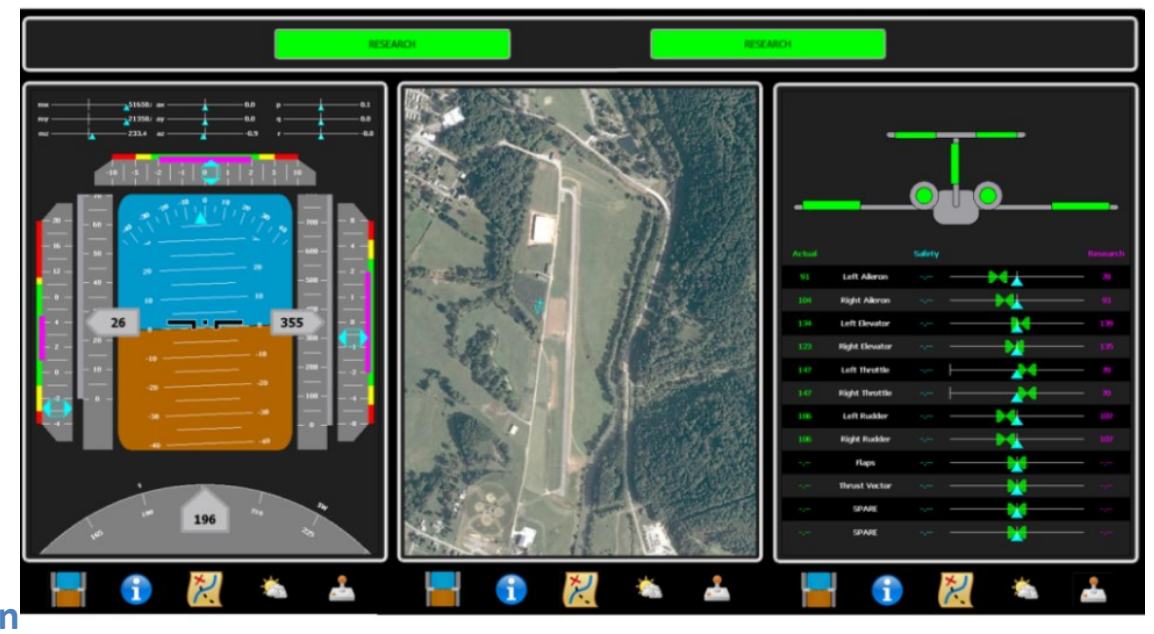

Figure 3.8. GCS display windows: Primary Flight, Overhead Map, and Surfaces Displays

\subsubsection{Central Computer}

The central computer in the the GCS is tasked with all computing and visualization which includes the following functions:

1. Receives data downlinked from the UAV through serial communication with the wireless modem;

2. Receives input commands from the pilot control station;

3. Sends pilot commands to the wireless modem via serial communication;

4. Displays aircraft data through visualizations described in sect. 3.3.2.3;

5. Computes derived data for use in the synthetic vision. 


\subsubsection{Simulation Function}

\subsubsection{Capabilities}

The design objective for the WVU GCS simulator was to create a platform to be used for pilot training, testing of new software and human-machine interfacing, pilot modeling, and aircraft research. Figure 3.9 shows the communication protocol and setup for the simulator. First, it is essential to train pilots and test new functions for the GCS including new hardware and software updates. Pilot training can be conducted using a Phastball research UAV mathematical model $^{36}$ under a manual and augmented control mode (discussed in Section IV.B.2). Pilot modeling can be conducted through various flight scenarios that push the efforts of the pilot as well as modeling the ability of pilots with different flight experiences. Finally, research can be conducted for aircraft during extreme maneuvers, flight conditions in abnormal environment conditions, etc. Figure 3.10 shows the setup for the GCS simulator.

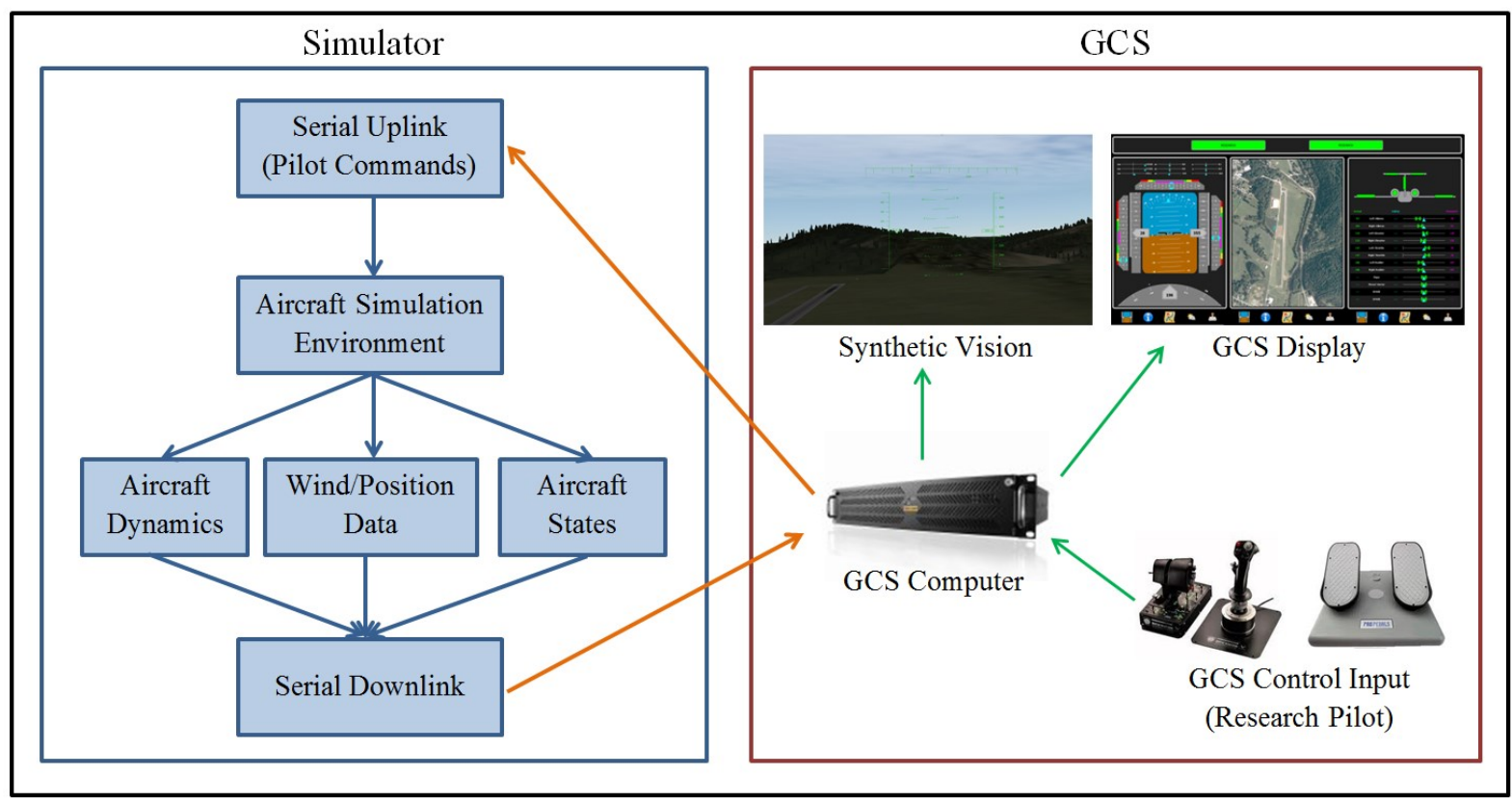

Figure 3.9. Simulator \& GCS Communication Protocol 


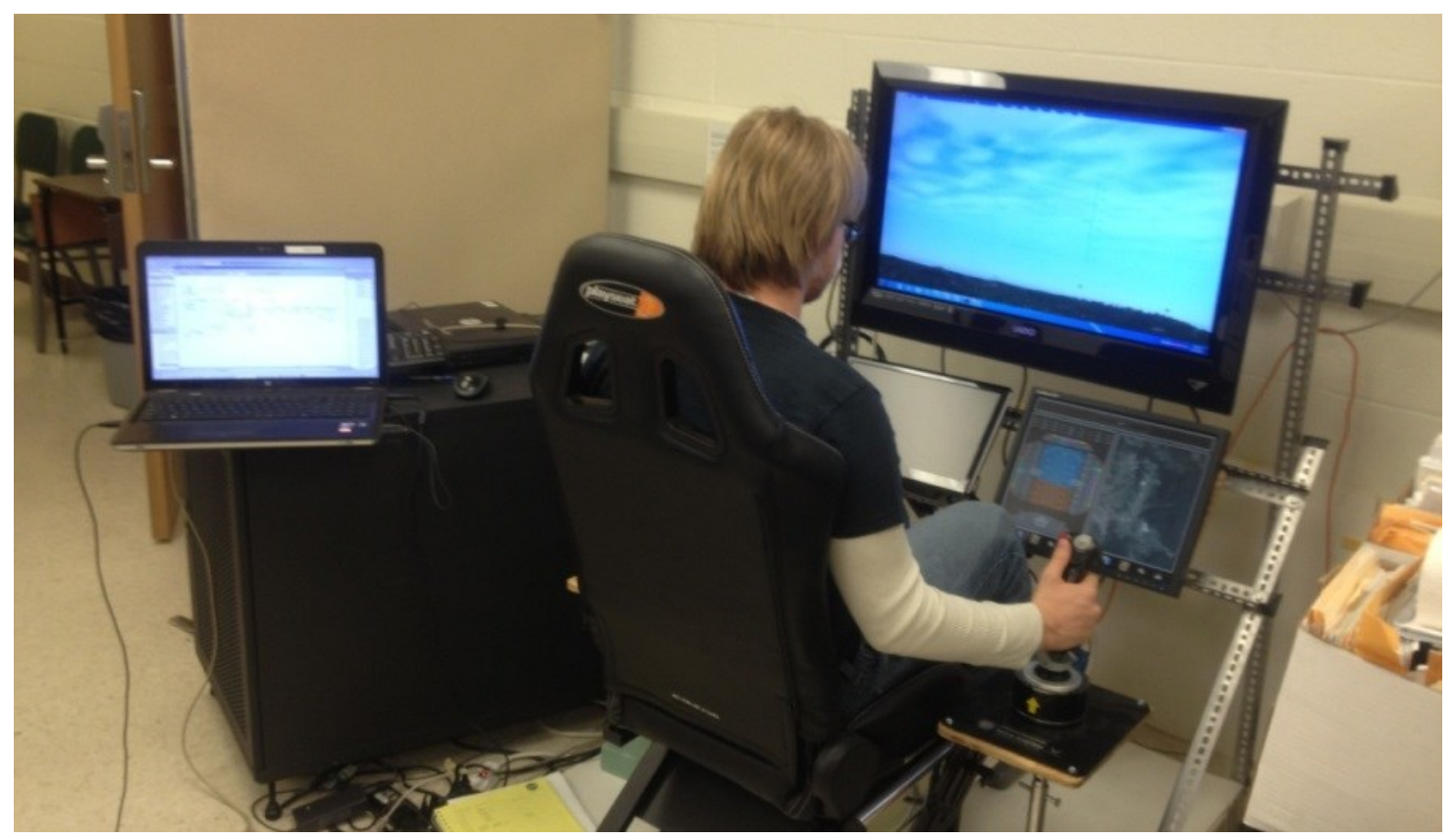

Figure 3.10. WVU GCS Simulator Setup in Lab

\subsubsection{Design}

The simulator of the Phastball research UAV was developed within a Matlab/Simulink environment. The general block diagram of the simulator is shown in Figure 3.11. The simulator is based on the open source freely available Flight Dynamics and Control (FDC) Toolbox ${ }^{37}$. Based on the results of the previous PID efforts ${ }^{36}$, the default Beaver standard aircraft dynamic model in the FDC was replaced with a non-linear dynamic model of the WVU Phastball research UAV. Additionally, a static and dynamic thrust model was developed and implemented into the simulator using experimental results. Serial communication is used for simulated uplink and downlink between the simulator and the GCS. The WVU Phastball research UAV simulator can implement both the manual and augmented control modes for the aircraft. These two types of control are discussed further in Section IV.B.2. 


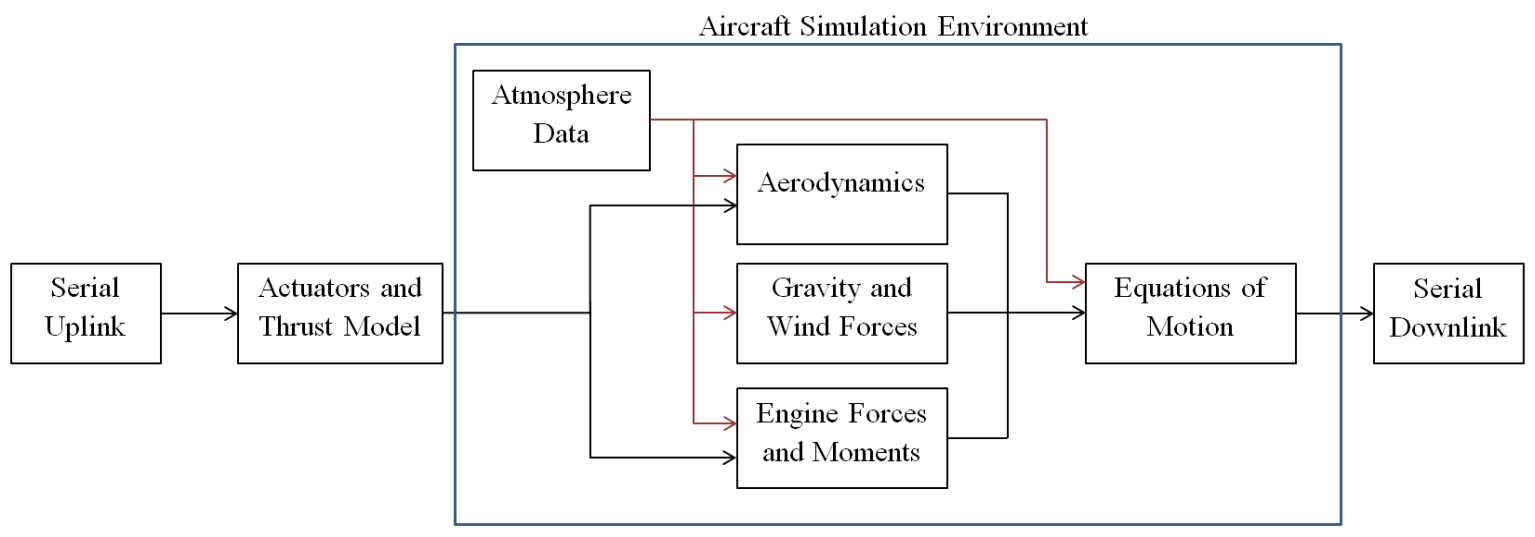

Figure 3.11. Conceptual Representation of Simulator Designed in Simulink

\subsubsection{Benchmark Maneuvers for Simulation}

In order to validate the simulator certain "Benchmark" maneuvers were performed that would accurately depict each of the desired research areas for the GCS.

Table 3.4. Basic Flight Maneuvers

\section{Airplane Basic Flight Maneuvers}

\section{Maneuvers}

\section{Rectilinear Flight}

Approach to Stall

Unusual Attitudes and

\section{Recoveries}

\section{Basic Flight Patters}

Straight Climbs and Descents

\section{Turns}

Maintain Constant Airspeed, Altitude, and Attitude

Takeoff Configuration, Clean Configuration, Approach or Landing Configuration

Nose-High Attitudes, Nose-Low Attitudes

Racetrack, Standard Procedure Turn, 80/260 Procedure Turn, Teardrop Pattern, Circling Approach

Entry and Leveling Off for both Climbs and Descents

Standard-Rate, Turns to Predetermined Headings, Timed,

Compass, Steep (excess of normal instrumented turns),

Climbing, Descending

1. Piloted Aircraft Flight: In order to simulate instrumented flight in piloted aircraft it is 
important to perform certain types of maneuvers. These maneuvers come from the Instrument Flying Handbook written by the U.S. Department of Transportation Federal Aviation Administration ${ }^{38}$ and they involve the ability for attitude and power control and are shown in Table 3.4.

2. Pilot Modeling: In order to accurately model pilot behavior it is important to have the pilot fly under scenarios that replicate flight conditions used for real flight simulation and research purposes. This includes flying under a time delay in order to look at how the delay in control affects the pilot's ability to perform maneuvers and stabilize the aircraft. Flying with limited rates on surfaces or having the pilot fight maneuvers from the flight computer such as doublets performed on various channels can show pilot learning under adverse conditions as well as research how they compensate for these changes.

3. Aircraft Modeling: For aircraft modeling it is important to excite each mode of the aircraft. Flying doublets as well as performing 'multisines' on each control surface helps in developing accurate models through parameter identification. Flying the aircraft at high angles of attack and performing extreme maneuvers will give a gauge on how well the aircraft will react as well its physical limitations in flight.

4. Surveillance: Simulating surveillance missions require long duration flights that encircle a desired area of importance. The GCS can implement an autopilot mode for flying certain patterns such as circles or figure eights over designated coordinates. Data can be sent to the GCS in real time allowing human interaction and decision making.

\subsubsection{Flight Test Function}

\subsubsection{Design Objectives}

In order to qualify the GCS as operational for research, it had to function as well as or better than $\mathrm{R} / \mathrm{C}$ flight. Therefore, the research pilot was instructed to fly a series of maneuvers in order to replicate $\mathrm{R} / \mathrm{C}$ flight for a comparison to previous research capabilities. These maneuvers included pitch and roll doublets, coordinated turns, and GCS control of an entire flight lap. Once the pilot was comfortable with his ability to complete the doublets he 
proceeded to coordinated turns and finally to the complete flight lap.

\subsubsection{Control Modes}

Three types of operating modes were developed to control the WVU Phastball research UAV from the GCS:

1. Manual flight mode

In the manual mode, the GCS pilot sends commands directly to the five control surfaces and the throttle through the movement of the ground joystick. In this mode the GCS is in full control of the aircraft.

2. Augmented Flight Mode

The augmented flight mode has the ability for tracking desired attitude angles as well as desired rates for roll and pitch. The GCS pilot controls desired values, either heading or rates, through the ground joystick. In this mode, he has direct control of the throttle settings while he does not have control over the rudder setting.

3. Autonomous flight mode

In autonomous flight mode the UAV has full control over the aircraft calculating desired attitude and throttle settings and maintaining those settings through the use of an inner loop controller. A detailed discussion on autonomous flight mode is discussed below.

In order to implement the augmented flight mode a set of Linear Quadratic Regulator (LQR) based control laws are running onboard of the UAV to convert desired attitude angles to servo movements. The object of the state regulator is to drive the initial condition error to zero, in this case the difference between desired and actual attitude. The gains for the controller were initialized through the controller design with the identified Phastball research UAV model, and then fine-tuned through flight testing. By default with no control input from the pilot, the aircraft will track a desired pitch angle of two degrees and a desired roll angle of zero degree which is based on typical angle-of-attack at cruise speed. Figure 3.12 shows the design architecture for the augmented flight mode during attitude tracking. 


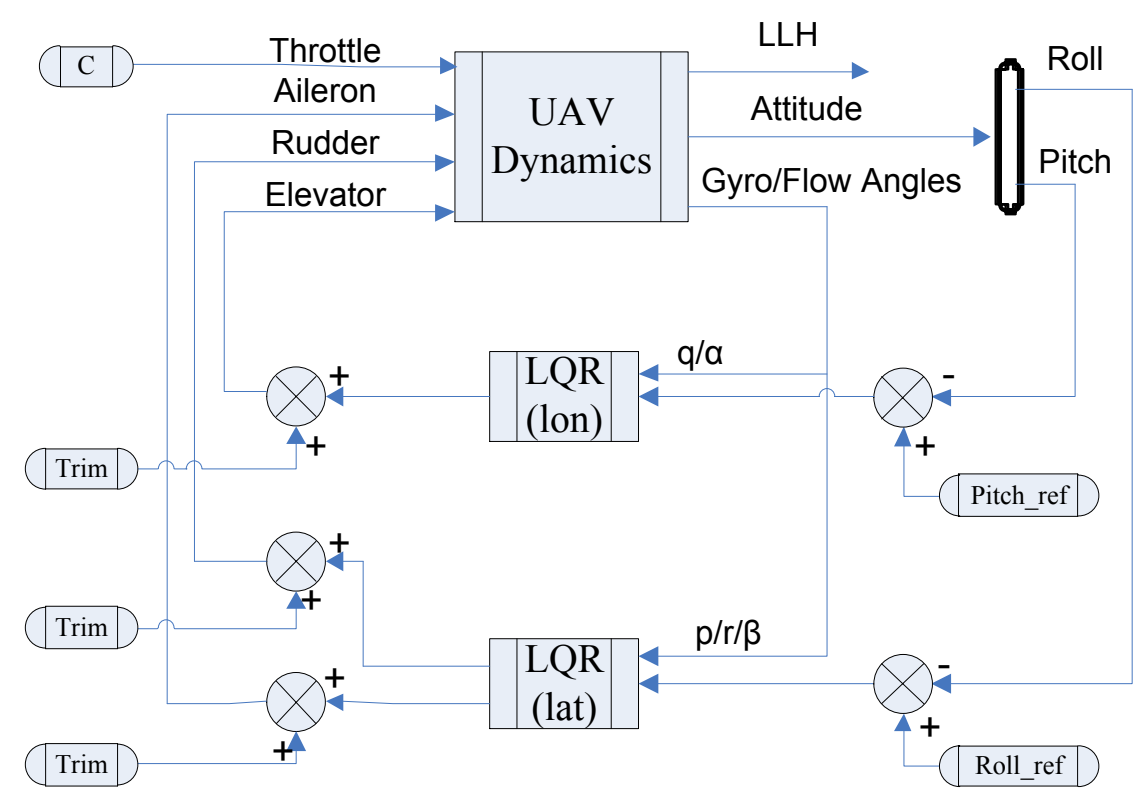

Figure 3.12. Overview of Augmented Flight Control.

\subsubsection{Benchmark Maneuvers for Flight Testing}

For flight testing the same maneuvers identified for the simulator, section 3.3.3.3, are applicable for the verification of the GCS in flight testing.

\subsubsection{Communication}

Extensive flight tests were conducted during the 2011 and 2012 flight testing seasons to validate and improve the performance of the GCS. Different hardware models, locations, and settings were investigated on the GCS vehicle to reach satisfying results; this is the case of the transreceiver and its antenna were investigated through both ground and UAV flight tests to reach satisfying results.

Both downlink and uplink have been thoroughly tested through UAV flight tests. A high level of reliability was reached for the downlink communication part, and the corrupted and lost packets are less than $0.2 \%$ when flying under typical conditions. For the uplink communication, the sum of corrupted and lost packets reaches a maximum packet loss of $38 \%$ during a onesecond window however the total packet loss was only $1.94 \%$ for the entire flight. Figure 3.13 shows the percentage of lost packets while the UAV was on the runway and during flight; note 
that flight takes place between approximately 200 and 500 seconds. Figure 3.14 shows the percentage of lost or corrupted uplink packets with respect to aircraft ground position, the plot on the right shows one lap while the plot on the left shows the entire flight.

It is observed that these maximum losses happen along the North portion of the lap as well as during high banked turns. This could be due to the relative positions of the UAV and GCS antennas as well as the increased distance between the UAV and GCS. Another possible reason for the data loss of the uplink is that GCS software does not always send in $50 \mathrm{~Hz}$, since the GCS computer is not running in real time. It is worth mentioning here that the uplink rate was observed to dramatically increase when synthetic vision via X-Plane was not running. Because of this issue, the synthetic vision was not utilized during flight tests. In the future it is planned to remedy this problem by running the synthetic vision on a secondary computer dedicated to the visual interface. Finally, it is important to note here that the total latency of the GCS is roughly 0.072 seconds or about 3.6 time steps.

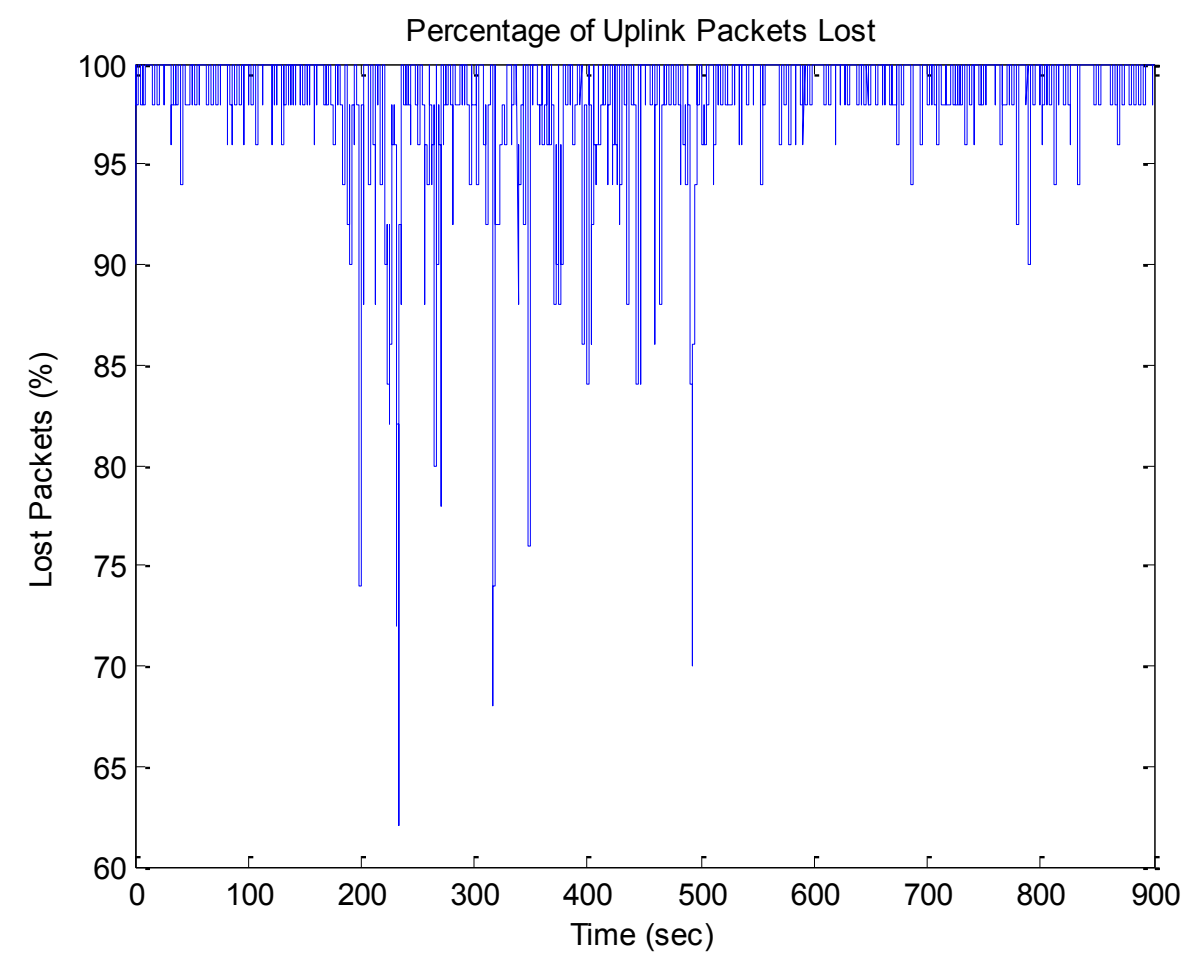

Figure 3.13. Uplink Rate (Packets per Second) 

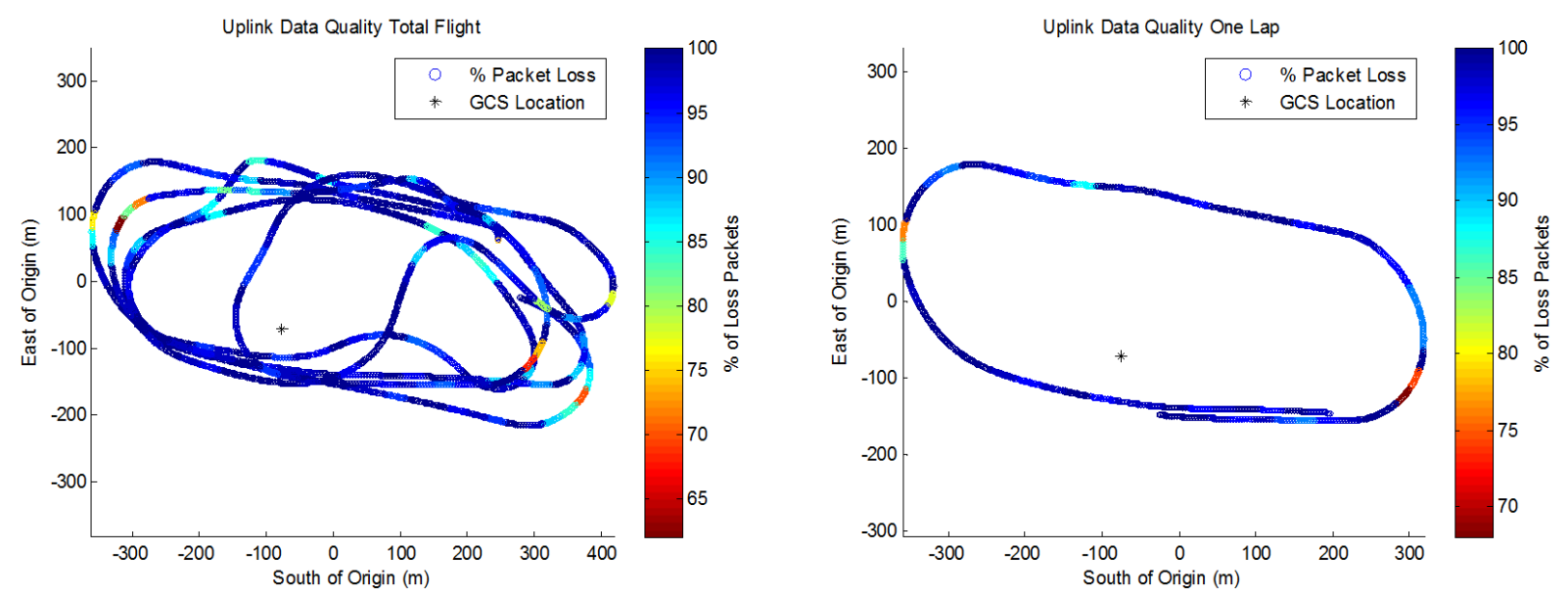

Figure 3.14. Uplink Packets Received During GCS Controller Flight

\subsubsection{Flight Monitoring}

The final major application of the GCS is for flight monitoring by downlinking any type of data that the researcher may want to look at during flight. This is helpful for two purposes; first, it allows for real time updates of what is happening in flight which is often useful for making sure the onboard schemes are running correctly. It is, in many cases, difficult to fully test a flight scheme in the lab or on the ground before flight and therefore, little more than simulations can be accomplished. Another difficulty to flight testing is that often the perspective of the pilot and researchers on the ground is quite different to that of a pilot in the cockpit. Therefore, ground monitoring of real time flight data can give a better indication of what is going on in flight.

Second, flight monitoring is a major advantage of any GCS used in civilian, military, or research applications. Often a pilot flying from a GCS or a program running onboard a GCS may need to make decisions based on a multiple pieces of data from flight and so quick, efficient monitoring of system critical flight data is necessary for mission success. Not only is it good for decision making but can also be a good useful warning system of possible failures as well as give insight into future problems. 


\section{Wind Sensing}

\subsection{Introduction}

Wind is a very unique and complex phenomenon of the atmosphere and has proven difficult to understand, model, and predict. Wind and wind gusts appear in nature as random events that very both spatially and temporally and, therefore, it is very difficult to know the local components of wind at any particular point and time. However, there has been lots a research into this area for many years and from this models have developed which help to simulate wind and predict future wind conditions.

These models include the Dryden and Von Karman turbulence models which are mathematical representations of wind gusts. These methods treat both the linear and angular velocity components of the wind as varying spatially according as a stochastic process. They also specify the power spectral densities which are used for both the linear and angular components of the gusts. The main difference between the two is that the Dryden model is characterized by rational power spectral densities while the Von Karman model is characterized by irrational ones. Generally the Von Karman model is used as it more closely represents actual atmospheric data ${ }^{39}$.

Even with the use of these models for design purposes they are not practical for local knowledge of the wind near the UAV. Because of this techniques need to be developed that will allow the UAV to estimate the wind in order to harness the wind for energy and to develop accurate warning signs for dangerous weather conditions. One of the current methods used to detect local wind states is the triangle method which used the difference in the airspeed vector and the GPS speed vector in order to estimate the wind vector. This section will look at the accuracy of this method as well as using a variant of the Kalman filter to estimate the wind conditions.

This chapter will begin by looking at how wind was modeled in the simulator for the development of wind estimation. Next, it will show how the truth measurement for wind was collected along with how the UAV collects data for wind estimation. Following that this chapter 
discusses how this technique compares with other ones used and how well it does within a flight testing environment. Finally, this chapter discusses the use of wind estimation for the use in gust suppression control where the aircraft will try to minimize vertical accelerations induced by gusts.

\subsection{Modeling}

For simulations used in wind estimation and wake modeling it is important to have a wind model that accurately depicted wind gusts seen in flight testing. The one minus cosine model is often used for its simplicity ${ }^{40}$. Each individual gusts consist of a cosine pulse, depicted in Figure 4.15 where $U_{0}$ is the amplitude of the idealized wind gust.

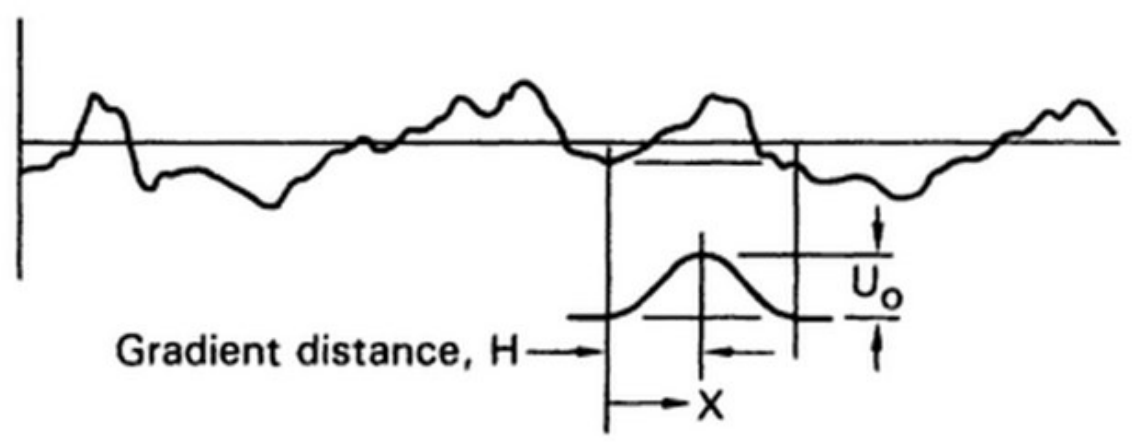

Figure 4.15 One minus cosine wind idealization (reproduced from 40)

\subsection{Phastball Research UAV Air Data System}

\subsubsection{Nose Board Hardware}

In order to collect air speed and flow angle data, a nose board was developed by the FCSL for the Phastball research UAV platform. A Netburner Mod5213 is used as the main processor which powers the sensors in the nose and sends the data back to the flight computer. Three low-friction inductive potentiometers are used for the measurement of left and right angle of attack as well as the sideslip angle. For pressure data, two sensors from Sensor Technics measure static and differential pressure. These pressure sensors were chosen with the requirements of a 2000 meter flight ceiling and a maximum flight speed of 60 meters per second. The processor communicates with the pressure sensors at $400 \mathrm{~Hz}$ via Serial Peripheral Interface (SPI) protocol. 
To reduce the effects of noise, an average filter is running every eight samples for both differential and static pressure data at $50 \mathrm{~Hz}$. A Pitot tube is installed in front of the aircraft for the collection of pressure data before the airflow is disturbed. All five air data sensors are calibrated and compensated for temperature effects. Figure 4.16 (a) shows the nose board of the ADS which collects the data from the Pitot tube and airflow sensors, Error! Reference ource not found. (b).

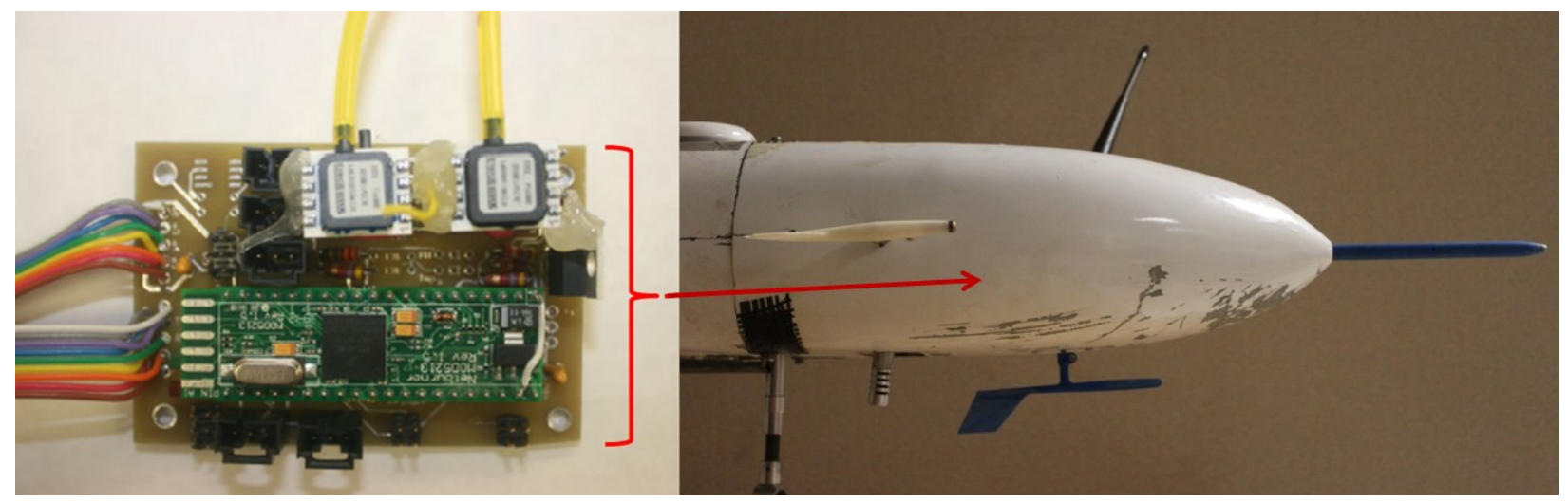

(a) Nose board

(b) Pitot tube and air flow sensors

Figure 4.16 Air Data System

Calibration of the nose board was done on a calm day with little to no wind. The airspeed of a UAV can be estimated from dynamic pressure using equation (4.1) where $k$ is the calibration constant which is due to misalignment error during the installation. $P_{d}$ is the differential pressure data in units of Pascal, and $\rho$ is air density measured in units of kilograms per cubic meter. Equation (4.2) is used to calculate the air density based on the static pressure, $\mathrm{P}_{\mathrm{s}}$, and the temperature, $\mathrm{T}$, of the surrounding air. Based on these two equations the optimal calibration constant, $\mathrm{k}$, is found by assuming no wind and solving the evaluation function given in equation(4.3). $V_{\text {Pitot }}$ is the airspeed sensed by the Pitot tube and is a function of $k, V_{G P S}$ is the ground speed for the UAV from the GPS sensor. $V_{\text {Pitot }}$ and $V_{G P S}$ are identical under a no wind condition so the expectation, $\mathrm{E}[\cdot]$, of the difference throughout the entire flight is zero. These calibration runs were conducted on a calm day in order to minimize the errors introduced from a non-uniform wind field. Finally, through this process the optimal value for $k$ was found to be 1.1149 , obtained through numerical solutions. 


$$
\begin{aligned}
& V_{\text {Pitot }}=k \sqrt{\frac{2 P_{d}}{\rho}} \\
& \rho=\frac{P_{s}}{287.058^{*} T} \\
& \min E\left(\left|V_{\text {Pitot }}-V_{G P S}\right|\right)
\end{aligned}
$$

Figure 4.17 and Figure 4.18 show the estimation results of air speed using the pressure data both before and after calibration respectively. Figure 4.17 shows a large difference between the raw Pitot tube data and the velocity calculated from GPS measurements. This is due to errors in the Pitot tube setup, as discussed above, and is the reason why calibration is needed in order to get useful data. Figure 4.18 shows that the airspeed and GPS speed match quite well after the calibration. The slight difference between Pitot tube and GPS data is due to the slight breeze during the flight test. As expected the sir speed is less than the GPS speed when flying with the wind and more when flying against the wind.

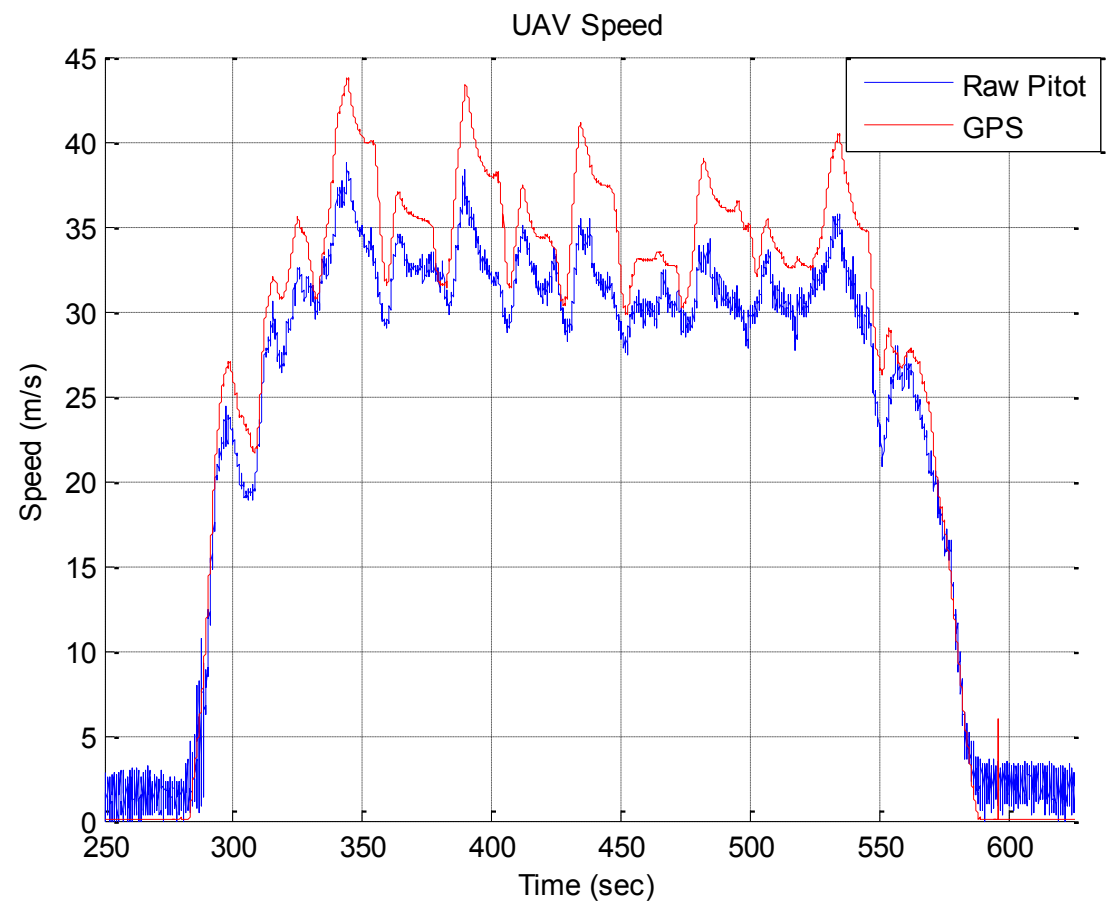

Figure 4.17 Raw air speed 


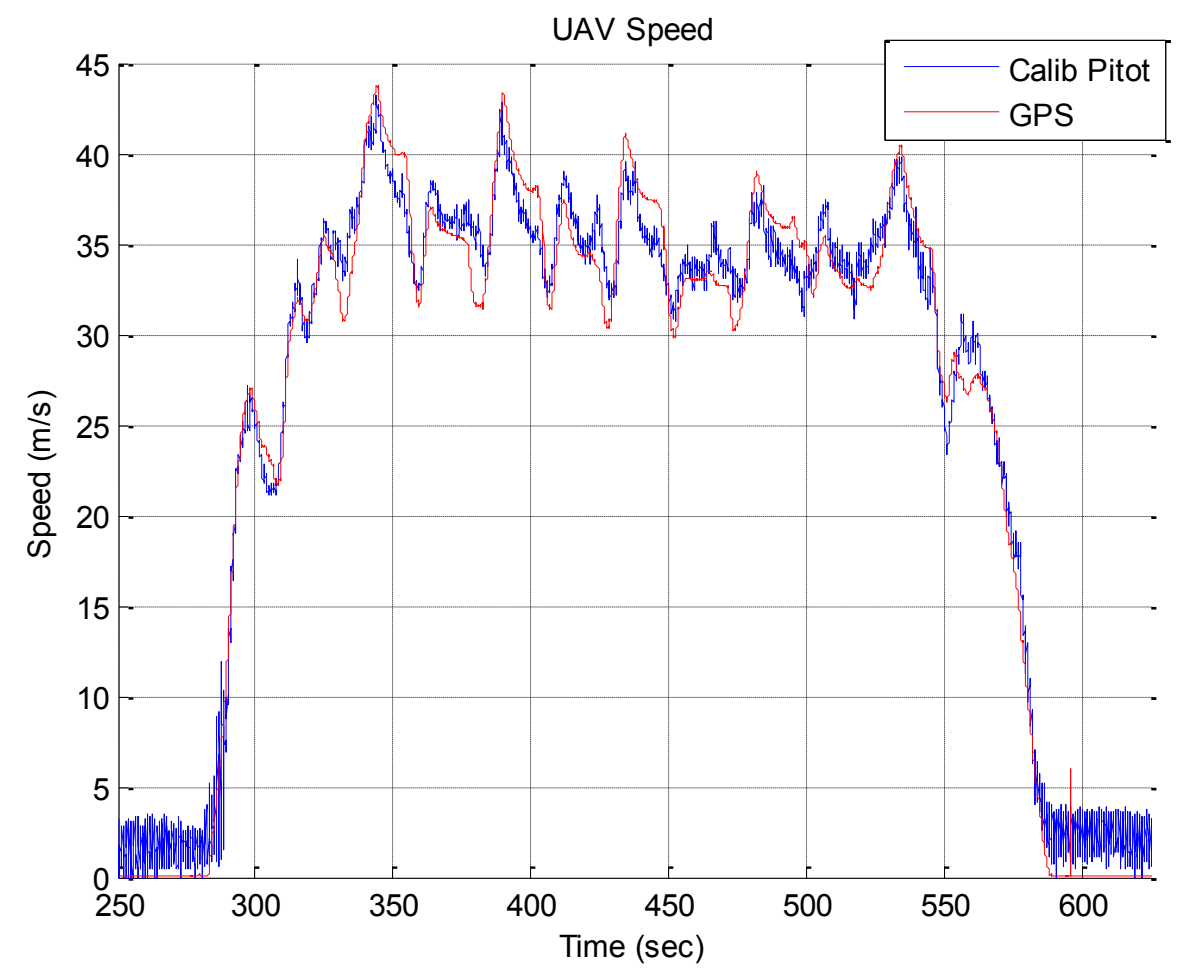

Figure 4.18 Calibrated air speed

\subsubsection{Weather Station for Ground Truth}

For validation of wind sensing methods it is important to have a ground truth, this was accomplished through various weather stations erected at the flight field. The weather station chosen for this application was the Ultimeter $2100^{41}$ from Pete Bros. shown in Figure 4.19. The specifications for this weather station can be seen in Table 4.5. Two weather stations were set up, the first was at the ground control station and the other was set up approximately 240 meters to the south. In order to minimize ground effects and nearby structures the weather stations were mounted on poles approximately 8 meters above the ground. 


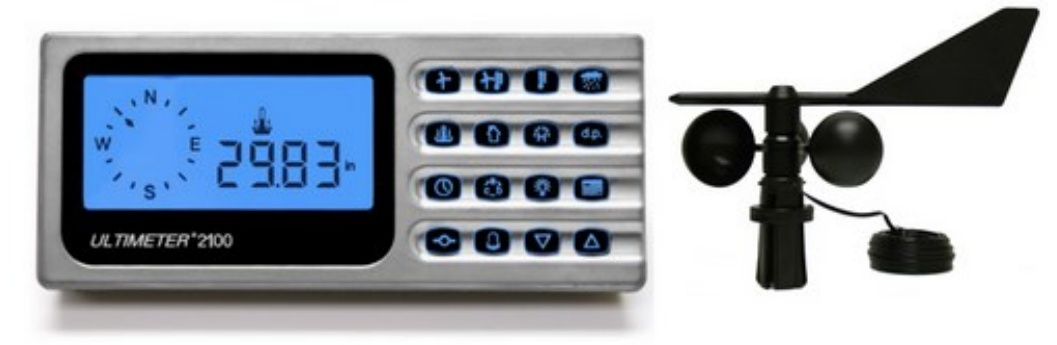

Figure 4.19 Pete Bros. Ultimeter 2100

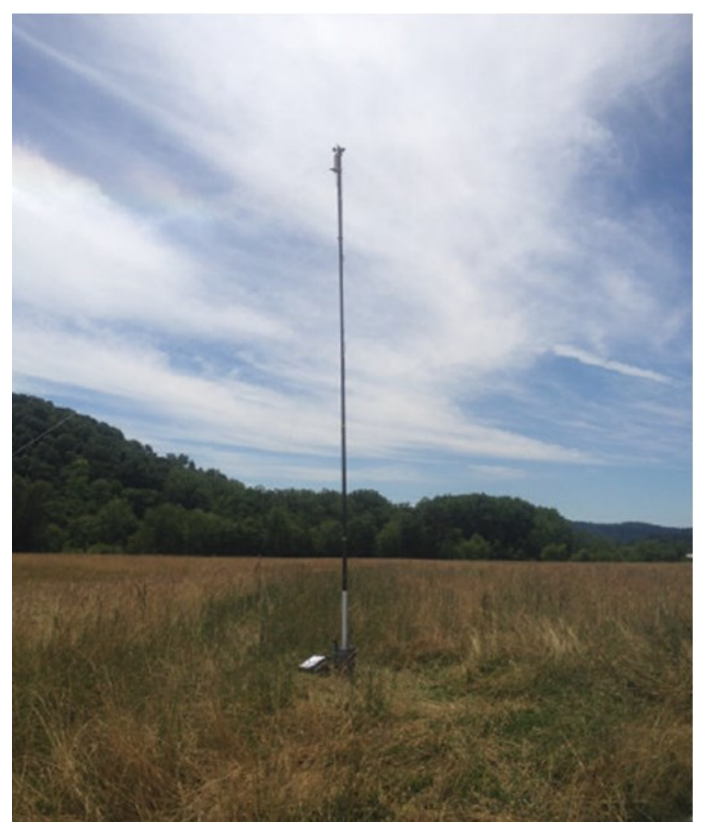

Figure 4.20 Weather station setup during flight testing

Table 4.5. Weather Station Specifications

\begin{tabular}{|c|c|c|c|}
\hline & & Spec & Value \\
\hline & & Threshold & $1.5 \mathrm{mph}$ \\
\hline (ऐ) & & Accuracy & $\pm 2 \mathrm{mph}$ or $\pm 5 \%$ (whichever is greater) \\
\hline 气 & & Resolution & $0.1 \mathrm{mph}$ \\
\hline$\stackrel{\frac{U}{2}}{<}$ & & Range & 0.2 to $160.0 \mathrm{mph}$ \\
\hline & & Accuracy & $\pm 5 \%$ full range \\
\hline 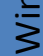 & $\stackrel{\frac{1}{\pi}}{>}$ & Resolution & $1 \mathrm{deg}$ \\
\hline
\end{tabular}




\subsection{Wind Estimation (UKF Development)}

Given GPS velocity, aircraft attitude, and both pressure and flow angles the wind speed can be estimated using an Unscented Kalman Filter ${ }^{42}$ in the following form:

$$
\begin{aligned}
& \text { l } \\
& y=h(x)+v, \quad \text { where } v \sim N(0, R)
\end{aligned}
$$

where: is the derivative of the states, $f(x, u)$ is the nonlinear function that relates the derivative of the states to the states and inputs of the system. $y$ is the output of the system, $h(x)$ is the nonlinear measurement model, and $v$ is the zero-mean Gaussian measurement noise with a covariance matrix of $R$. The UKF is an extension to the Kalman Filter that estimates the states of a nonlinear system through the idea that "it is easier to approximate a Gaussian distribution than it is to approximate an arbitrary nonlinear function or transformation" ${ }^{\prime 3}$. In other words, instead of sending the states through a nonlinear function for the prediction, a number of sigma points are selected that represent the mean and covariance of the states and the nonlinear function is applied to those sigma points. This leads to a cloud of transformed points that represents the statistics of the output of the system. The steps to the UKF are shown below in Table 4.6 and are as follows:

1. Prediction of the state and its covariance matrix

a) Sigma points are calculated as a weighted set of points based on the mean and covariance of the system's states

b) Each of these sigma points along with the inputs to the system are passed through the process model

c) A predicted state's mean and covariance is computed

2. Prediction of the expected observation and its innovation covariance matrix

a) Prediction points are calculated by passing the sigma points and along with the system's inputs through the observation model

b) A prediction of the observation is calculated based on the prediction points 
c) The innovation covariance is calculated using with the introduction of the measurement noise

3. Prediction of the cross-correlation matrix

Table 4.6 Update and measurement equations for the UKF

\begin{tabular}{|c|c|}
\hline Time Update Equations & Measurement Update Equations \\
\hline 1. Initialize & $\begin{array}{l}\text { 1. Choose Sigma Points using Current Best Guess } \\
\text { for State and Covariance }\end{array}$ \\
\hline $\begin{array}{c}x_{0}^{+}=E\left(x_{0}\right) \\
P_{0}^{+}=E\left[\left(x_{0}+x_{0}^{+}\right)\left(x_{0}+x_{0}^{+}\right)^{T}\right]\end{array}$ & $\begin{array}{ll}x_{k}^{(i)}=x_{k}^{+}+x^{(i)}, & i=1, \ldots, 2 n \\
x^{(i)}=\left(\sqrt{n P_{k}^{+}}\right)_{i}^{T}, & i=1, \ldots, n \\
x^{(n+i)}=-\left(\sqrt{n P_{k}^{+}}\right)_{i}^{T}, & i=1, \ldots, n\end{array}$ \\
\hline 2. Choose Sigma Points & $\begin{array}{l}\text { 2. Transform Sigma Points using Nonlinear } \\
\text { Measurement Equation } h(\cdot)\end{array}$ \\
\hline$x_{k-1}^{(i)}=x_{k-1}^{+}+x^{(i)}, \quad i=1, \ldots, 2 n$ & $y_{k}^{(i)}=h\left(x_{k}^{(i)}, t_{k}\right)$ \\
\hline$x^{(i)}=\left(\sqrt{n P_{k-1}^{+}}\right)_{i}^{T}, \quad i=1, \ldots, n$ & 3. Estimate Predicted Measurement \\
\hline$x^{(n+i)}=-\left(\sqrt{n P_{k-1}^{+}}\right)_{i}^{T}, \quad i=1, \ldots, n$ & $y_{k}^{-}=\frac{1}{2 n} \sum_{i=1}^{2 n} y_{k}^{(i)}$ \\
\hline $\begin{array}{l}\text { 3. Transform Sigma Points using Nonlinear } \\
\text { System Function } f(\cdot)\end{array}$ & 4. Estimate Covariance of Predicted Measurement \\
\hline$x_{k}^{(i)}=f\left(x_{k-1}^{(i)}, u_{k}, t_{k}\right)$ & $P_{y}=\frac{1}{2 n} \sum_{i=1}^{2 n}\left(y_{k}^{(i)}-y_{k}\right)\left(y_{k}^{(i)}-y_{k}\right)^{T}$ \\
\hline 4. Estimate a priori State & 5. Estimate Cross Covariance Between $x_{k}^{-}$and $y_{k}^{-}$ \\
\hline$x_{k}^{-}=\frac{1}{2 n} \sum_{i=1}^{2 n} x_{k}^{(i)}$ & $P_{x y}=\frac{1}{2 n} \sum_{i=1}^{2 n}\left(x_{k}^{(i)}-x_{k}^{-}\right)\left(y_{k}^{(i)}-y_{k}\right)^{T}$ \\
\hline 5. Estimate a priori State Covariance Matrix & 6. Update the State Estimate \\
\hline$P_{k}^{-}=\frac{1}{2 n} \sum_{i=1}^{2 n}\left(x_{k}^{(i)}-x_{k}^{-}\right)\left(x_{k}^{(i)}-x_{k}^{-}\right)^{T}+Q_{k-1}$ & $\begin{array}{c}K_{k}=P_{x y} P_{y}^{-1} \\
x_{k}^{+}=x_{k}^{-}+K_{k}\left(y_{k}-y_{k}\right) \\
P_{k}^{+}=P_{k}^{-}-K_{k} P_{y} K_{k}^{T}\end{array}$ \\
\hline
\end{tabular}


The system states, inputs, and output are listed in equations (4.6)-(4.8) where $[u, v, w]$ is the air speed expressed in the body frame, $[\phi, \theta, \psi]$ are the Euler angles, roll, pitch, and yaw, respectively, and $\left[w_{N}, w_{E}, w_{D}\right]$ are the wind velocities in the North-East-Down (NED) inertial frame. For the inputs to the system $\left[a_{x}, a_{y}, a_{z}\right]$ are the body frame accelerations measured by the onboard IMU and $[p, q, r]$ are the angular rates also measured by the IMU. The outputs of the system are $\left[V_{x}, V_{y}, V_{z}\right]$, the ground velocities measured by the GPS receiver, and $\left[V_{P i t o t}, \alpha, \beta\right]$ consisting of the airspeed, angle of attack, and sideslip angle which are measured by the ADS.

The first step in the UKF is to develop the dynamics of the system, in this case equation (4.9) calculates the body axis velocities and equation (4.10) calculates the attitude. Equation (4.11) shows the Direct Cosine Matrix (DCM) which is basically a rotation matrix to transform body axis velocities into the inertial frame. Because wind is composed of turbulent air flow, it is by definition random. Therefore, a random walk model is used in order to predict future wind states $^{10}$.

Finally, in order to relate the UAV velocities to the wind velocities the output equations were developed, shown in equations (4.12)-(4.15) where $\left[V_{\text {Pitot }}, \alpha, \beta\right]$ is expressed as a function of $[u, v, w]^{44}$. Since the difference in GPS velocity and body axis velocity is due to the wind acting on the UAV, equation (4.12) rotates the body axis velocity into the inertial frame using the DCM and corrects for wind with the GPS velocity ${ }^{45}$.

$$
\begin{aligned}
& x=\left[u, v, w, \phi, \theta, \psi, W_{N}, W_{E}, W_{D}\right] \\
& u=\left[a_{x}, a_{y}, a_{z}, p, q, r\right] \\
& y=\left[V_{x}, V_{y}, V_{z}, V_{\text {Pitot }}, \alpha, \beta\right] \\
& {\left[\begin{array}{rr}
i & q w+a_{x} \\
\cdot & -r u+a_{y} \\
i & -p v+a_{z}
\end{array}\right]+\operatorname{DCM}(\phi, \theta, \psi)\left[\begin{array}{l}
0 \\
0 \\
g
\end{array}\right]}
\end{aligned}
$$




$$
\begin{aligned}
& {\left[\begin{array}{ccc}
i & \sin (\phi) \tan (\theta) & \cos (\phi) \tan (\theta) \\
i & \cos (\phi) & -\sin (\phi) \\
i & \sin (\phi) \sec (\theta) & \cos (\phi) \sec (\theta)
\end{array}\right]\left[\begin{array}{l}
p \\
q \\
r
\end{array}\right]} \\
& D C M(\phi, \theta, \psi)=\left[\begin{array}{ccc}
\cos (\theta) \cos (\phi) & \cos (\theta) \sin (\psi) & -\sin (\theta) \\
\sin (\phi) \sin (\theta) \cos (\psi)-\cos (\phi) \sin (\psi) & \sin (\phi) \sin (\theta) \sin (\psi)+\cos (\phi) o s(\psi) & \sin (\phi) \cos (\theta) \\
\cos (\phi) \cos (\theta) \cos (\psi)+\sin (\phi) \sin (\psi) & \cos (\phi) \sin (\theta) \sin (\psi)-\sin (\phi) \cos (\psi) & \cos (\phi) \cos (\theta)
\end{array}\right] \\
& {\left[\begin{array}{c}
V_{x} \\
V_{y} \\
V_{z}
\end{array}\right]=\operatorname{DCM}(\phi, \theta, \psi)\left[\begin{array}{c}
u \\
v \\
w
\end{array}\right]+\left[\begin{array}{c}
W_{N} \\
W_{E} \\
W_{D}
\end{array}\right]} \\
& V_{\text {pitot }}=u \\
& \alpha=\tan ^{-1}\left(\frac{w}{u}\right) \\
& \beta=\sin ^{-1}\left(\frac{v}{\sqrt{u^{2}+v^{2}+w^{2}}}\right)
\end{aligned}
$$

\subsection{Simulation Results}

For simulation of wind estimation and gust suppression control the simulator described in section 3.3.3 was used for simulating the effects of wind on the UAV and using the output of the simulation for estimating wind states. The UKF described above (sect. 4.4) was used for estimating the wind states based on the response of the UAV within the FDC simulation environment.

\subsubsection{Wind Estimation}

Figure 4.21, below shows the UKF estimation of wind while flying through a constant wind field. It can be observed that the filter converges in less than 15 seconds and maintains its accuracy for the rest of the simulation. The top plot shows the estimation of the wind along each axis together with the truth data while the bottom plot shows the total calculated wind along with the truth. The total wind estimate is simply the norm of the wind states using equation(4.16). 


$$
w_{\text {tot }}=\sqrt{W_{N}^{2}+W_{E}^{2}+W_{D}^{2}}
$$

As shown in Figure 4.21, the UKF does a much better job at measuring the wind along the East axis. This is due to the fact that the observability of the problem changes when the wind expressed in the body frame changes. For example Figure 4.22 shows the UKF estimation of the same wind field except that the UAV is flying East instead of North. In this case the estimation of the wind along the North axis is much better than the other two and larger errors are observed along the East axis. It also appears in this case that the filter converges within about 5 seconds.

Finally Figure 4.23 shows the UKF estimation of a sinusoidal wind field where like before the wind estimation along the East axis is relatively good while the estimation along the North axis is not as good and in this case the wind estimation along the Down axis is the worst. Again the accuracy of the estimation of wind along each axis could be increased by changing the heading of the UAV. The bottom plot of Figure 4.23 shows the total wind estimation, again using equation (4.16), here the filter stays within $0.5 \mathrm{~m} / \mathrm{s}$ for the entire flight. and converges as soon as the UAV flies into the wind field at 5 seconds into flight. 

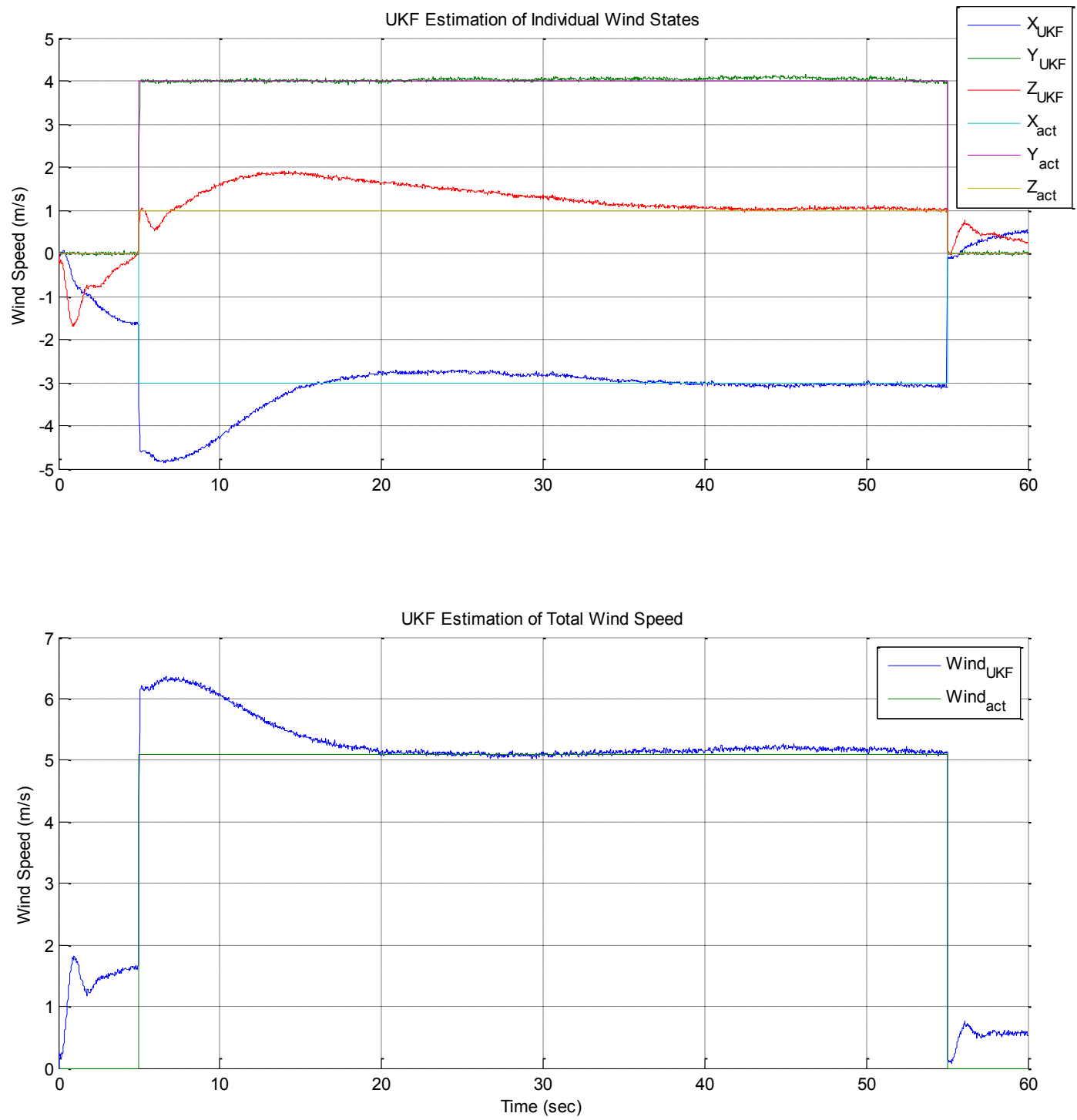

Figure 4.21 UKF estimation of wind for a constant wind field 

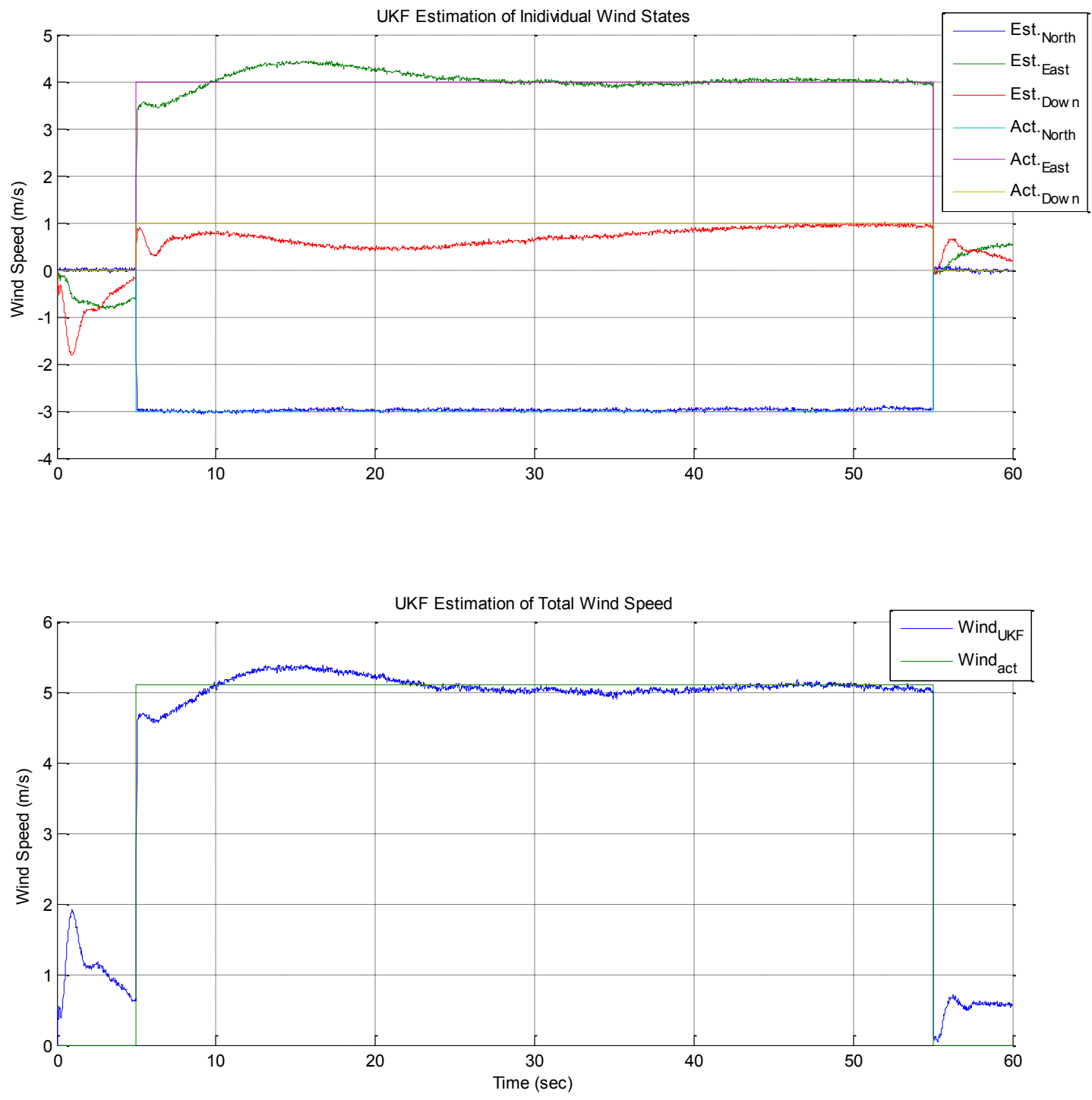

Figure 4.22 UKF estimation of wind for a constant wind field 

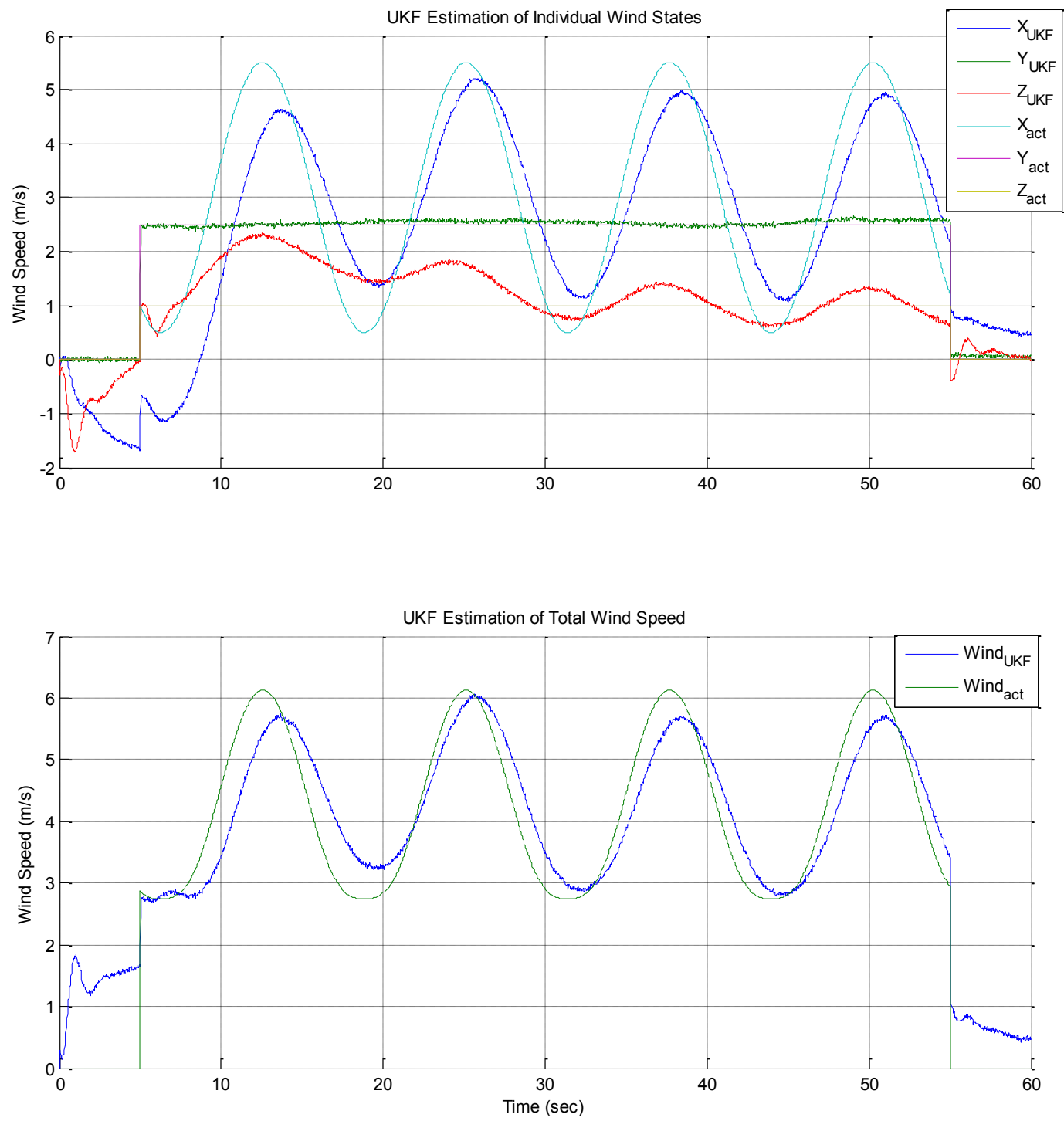

Figure 4.23 UKF estimation of wind for sinusoidal wind 


\subsection{Wind Estimation Method Comparison}

Wind estimation can be accomplished a number of ways including a direct calculation of the wind states from the dynamics of the aircraft as discussed in $[10,45]$ or estimation of the wind states through a UKF as discussed in section 4.4. Direct wind calculation methods use the wind triangle method to directly pull out the wind data. Therefore, the direct methods use equations (4.12)-(4.15), and just subtracts the body velocity from the inertial velocity to get wind states, shown in equation (4.17). The idea of the wind triangle can be seen in Figure 4.24 where the wind velocity is found as the difference between the airspeed and GPS speed.

$$
\left[\begin{array}{l}
W_{N} \\
W_{E} \\
W_{D}
\end{array}\right]=\left[\begin{array}{c}
V_{N} \\
V_{E} \\
V_{D}
\end{array}\right]-T^{-1}\left[\begin{array}{l}
u \\
v \\
w
\end{array}\right]
$$

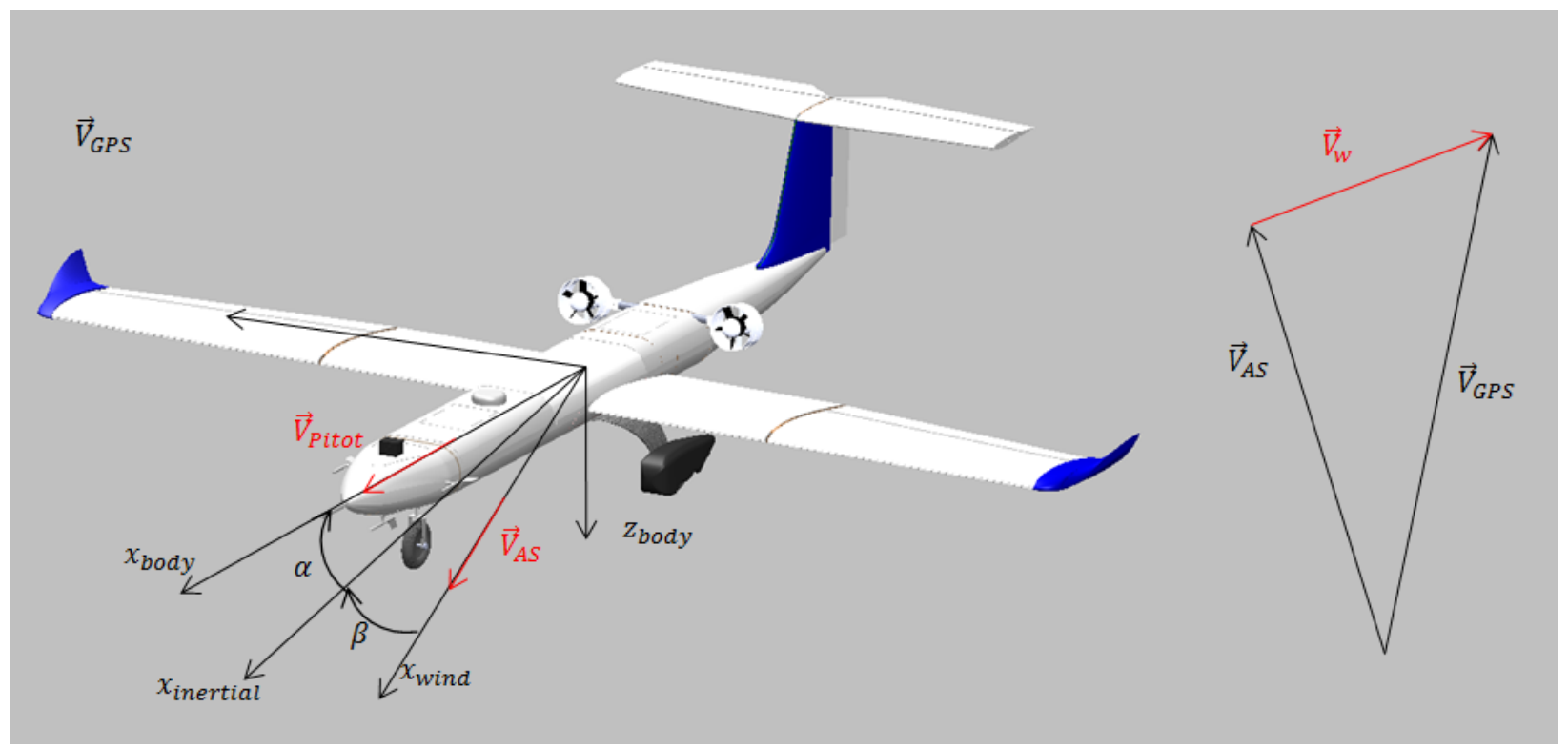

Figure 4.24 Wind triangle for direct method wind calculation

Shown in Figure 4.25 is a comparison of the wind estimation using the UKF and a direct calculation method described in [45]. For this simulation the wind state in the inertial frame was a constant wind field shown in Figure 4.21. A second simulation using a sinusoidal wind field shown in Figure 4.23 was conducted with the results shown in Figure 4.26. These simulations had added noise on the sensor outputs as described in section 3.2.2.2. 
Table 4.7 shows the statistical results of using both the UKF and the direct method to estimate the wind states. As shown the UKF has an improved standard deviation of 87 percent for a constant wind field after the UKF has converged and an increase of 19 percent for a sinusoidal wind field.

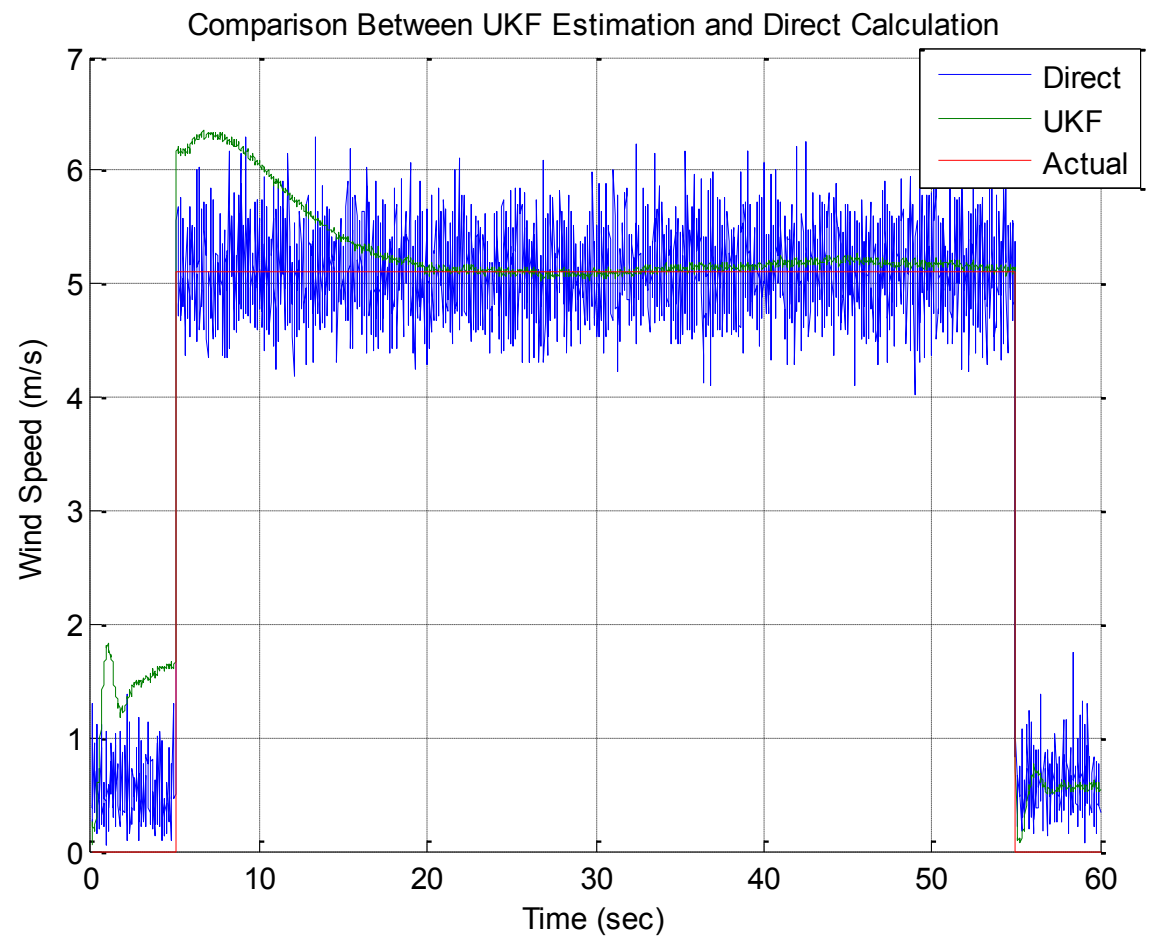

Figure 4.25 Wind estimation comparison between UKF and direct calculation 


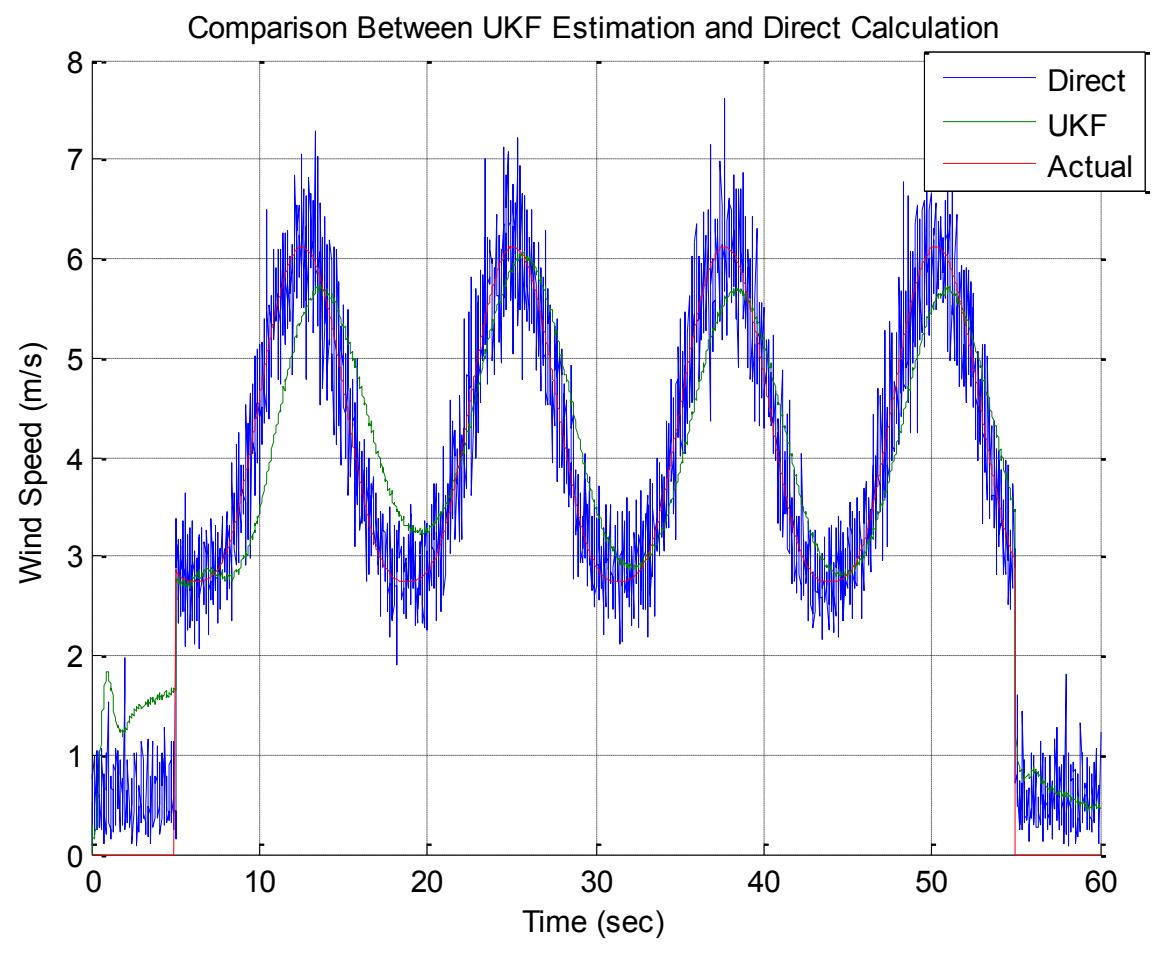

Figure 4.26 Wind estimation comparison between UKF and direction calculation

Table 4.7 Wind estimation comparison statistics

\begin{tabular}{lll}
\hline Wind Type & $\begin{array}{l}\text { Constant Wind } \\
\text { Field }\end{array}$ & $\begin{array}{l}\text { Constant/Cosine } \\
\text { Wind field }\end{array}$ \\
\hline $\mathrm{X}$ & -3 & $2 \cos (0.5)+3$ \\
$\mathrm{Y}$ & 4 & 2.5 \\
$\mathrm{Z}$ & 1 & 1 \\
\hline Statistics & & \\
\hline UKF std & 0.0988 & 0.6382 \\
Direct std & 0.7864 & 0.7899 \\
\hline
\end{tabular}




\subsection{Flight Results}

\subsubsection{Wind Estimation}

Due to the fact that the weather station stands about 8 meters off of the ground it has errors due to its proximity to the ground and therefore has to be corrected to compensate for ground effect. This is depicted below in Figure 4.27 which shows a smoke test using an experiment wind turbine that shows the wind at higher altitudes moving faster than wind closer to the ground.

Correcting the wind based on altitude is accomplished through the wind profile power law relationship ${ }^{10,46}$ shown in equation (4.18) where $W_{\text {corr }}$ is the corrected wind, $W_{\text {uncorr }}$ is the uncorrected wind measured by the weather station, $p$ is the power law exponent which is assumed to be approximately $1 / 7$ for neutral stability, $Z$ is the altitude of the UAV, and $Z_{r}$ is the reference altitude or the altitude of the weather station.

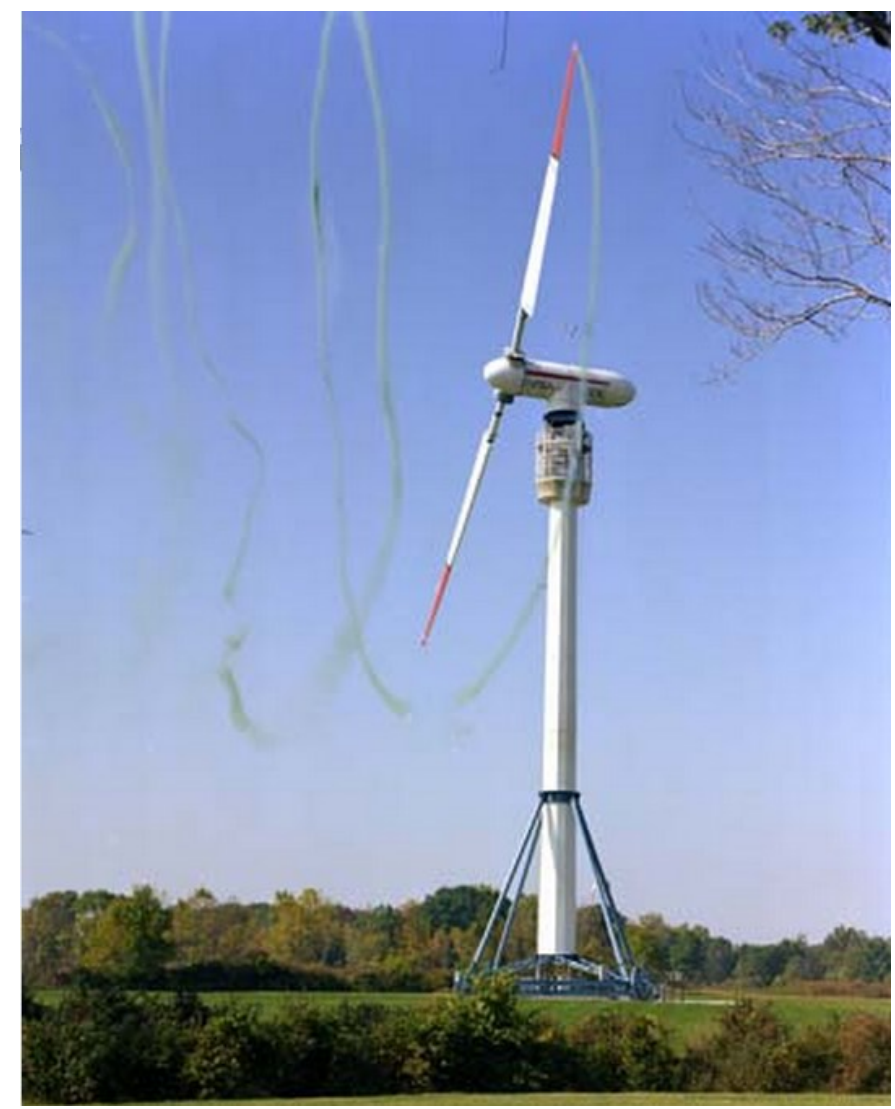

Figure 4.27 Wind speed at varying altitude (reproduced from NASA Glenn Research Center) 
The direction of the estimated wind is found using equation (4.19) where the atan2 function handles which quadrant the wind is coming from and $\left[W_{E}, W_{N}\right]$ is the measured wind along the East and North axis respectively. It is important to note that the angle of the wind is only in the North/East plane because that is the plane that the weather station measures the wind direction in.

As shown in Figure 4.28 the UKF wind estimation is generally within $1 / 2 \mathrm{~m} / \mathrm{s}$ to the truth given from the ground station while the wind direction estimation is within 25 degrees of the truth. There are a few parts of the estimation where the UKF is a bit off of the weather station, for example around 475 and 630 seconds. This is most likely due to the fact that wind changes spatially and since the UAV flies a lap and the weather station is stationary there can be a large distance of up to 500 meters between the two.

$$
\begin{aligned}
& W_{\text {corr }}=W_{\text {uncorr }} *\left(\frac{z}{z_{r}}\right)^{p} \\
& \psi_{w}=\operatorname{atan} 2\left(W_{E}, W_{N}\right)
\end{aligned}
$$



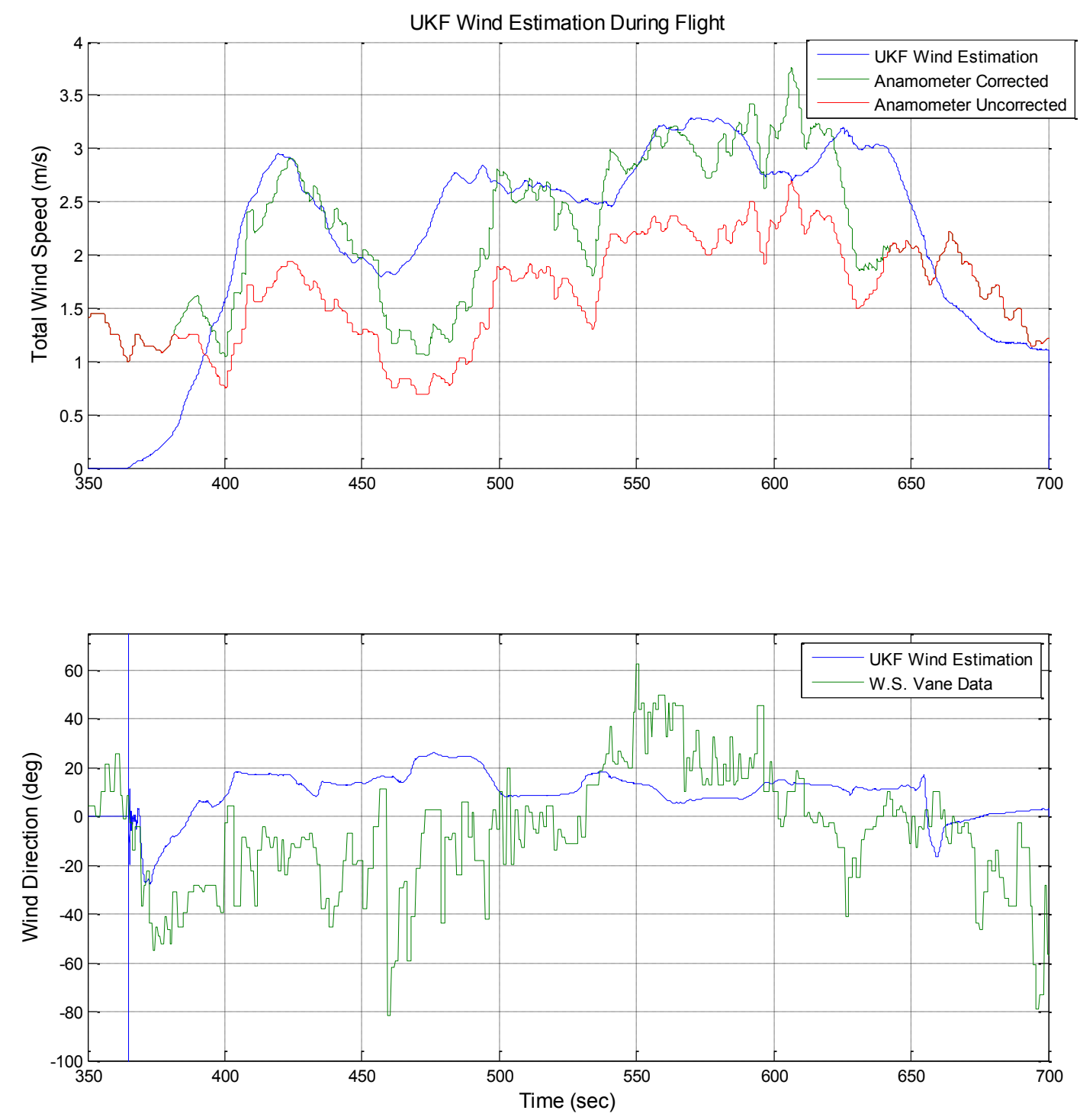

Figure 4.28 UKF wind estimation and weather station data 


\subsection{Gust Suppression Control}

One of the great advantages of knowing the wind states at the position of the leader is that the follower can compensate for the effects that the wind gusts will have on its dynamics. Strong wind gusts can induce large accelerations along the $z$ axis which can lead to aircraft damages and personal injuries. In order to alleviate these effects of the wind an elevator command is added on top of the command coming from the OLC to compensate for the wind gusts.

Simulations of gust suppression control shown below were conducted by flying the follower UAV through the wake of the leader. This can be done since the effect of the wake on the follower is simply an induced wind which can be looked at as induced wind gusts if they take place over short period of time. Additionally, it is important to be able to suppress abrupt changes due to wake encounters if formation flight will ever extend to human flight.

Figure 4.29 shows the induced wind onto the follower UAV from the leader's wake as described in sect.0. As shown there is only induced wind along the body $Y$ and $Z$ axis of the follower aircraft because the wake vortex is assumed to be perpendicular to the motion of the leading aircraft. As the follower aircraft moves through the wake, it can reach a significant roll angle. Such induced wind along the $Y$ and $Z$ axes can induce acceleration along the local $z$ axis of the airplane. Therefore, it is important to control the gust based on both the wind speed and acceleration of the wind along those two axes.

Equation (4.20) shows how the change in elevator is calculated based on the amplitude of the induced wind as well as the sign of the vertical position relative to the leader $\left(\zeta_{1}\right)$ and the sign of the roll angle of the follower $\left(\zeta_{2}\right)$. The direction the elevator needs to move is dependent on the roll angle of the UAV. If the UAV is encountering the same wind the elevator will need to move opposite if it is rolled left than if it was rolled right to decrease vertical accelerations. Likewise if the follower is above the leader than the horizontal induced wind is opposite and the elevator sign needs to flip once again. Equations (4.21) and (4.22) show the logic in flipping the sign of the elevator command as a function of leader and follower positions. 
It was found that for the results shown in Figure 4.30 and Figure $4.31\left[k_{1}, k_{2}, k_{3}, k_{4}\right]$ were found to be $[-4,-2,-1,-0.5]$.

Figure 4.30 shows the acceleration of the follower due to an encounter with the leader's wake both with and without gust suppression. Furthermore Figure 4.31 shows a more detailed plot of the accelerations along the $z$ direction of the follower UAV. Finally Table 4.8 shows the statistics of simulated gust suppression control which refers to the simulation of the previous two plots. As shown the maximum vertical acceleration induced by the wake was almost $16 \mathrm{Gs}$ while during gust suppression the maximum vertical acceleration drops to less than $1 / 3 \mathrm{Gs}$ which is much less noticeable.

$$
\begin{aligned}
& \delta_{E}=\left(\zeta_{1}+\zeta_{2}\right) *\left(k_{1} W_{E}+k_{3} \cdot\right. \\
& \zeta_{1}=-1 \quad P_{D F}<P_{D L} \\
& \zeta_{2}=-1 \quad \phi_{F}<0
\end{aligned}
$$

\section{Table 4.8 Gust suppression control statistics}

\begin{tabular}{lll}
\hline Statistics & No Gust Suppression Control & Gust Suppression Control \\
\hline Mean & -0.018 & 0.0031 \\
std & 1.7427 & 1.0 \\
Max & 15.9639 & 2.8185 \\
\hline
\end{tabular}




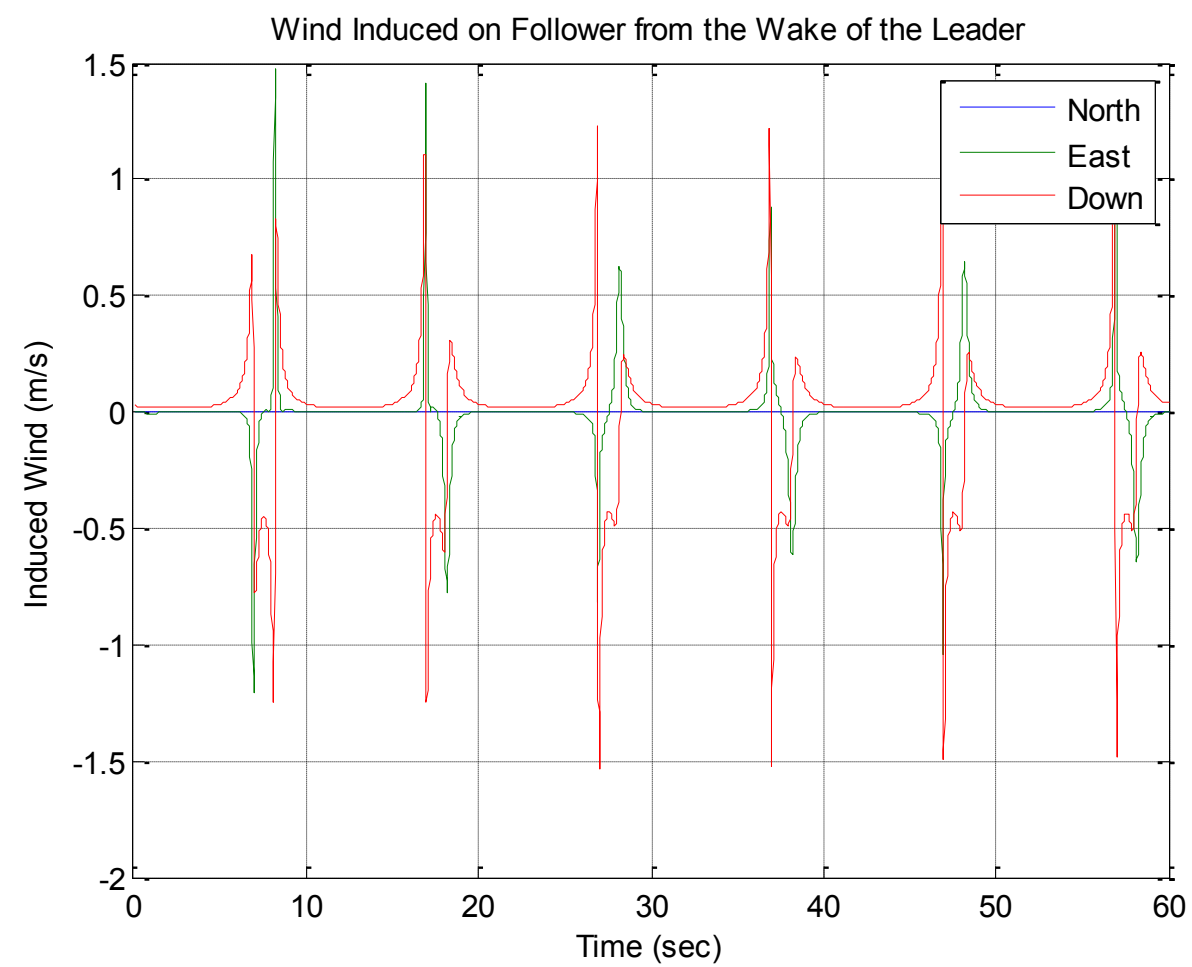

Figure 4.29 Induced wind from the leader's wake onto the follower

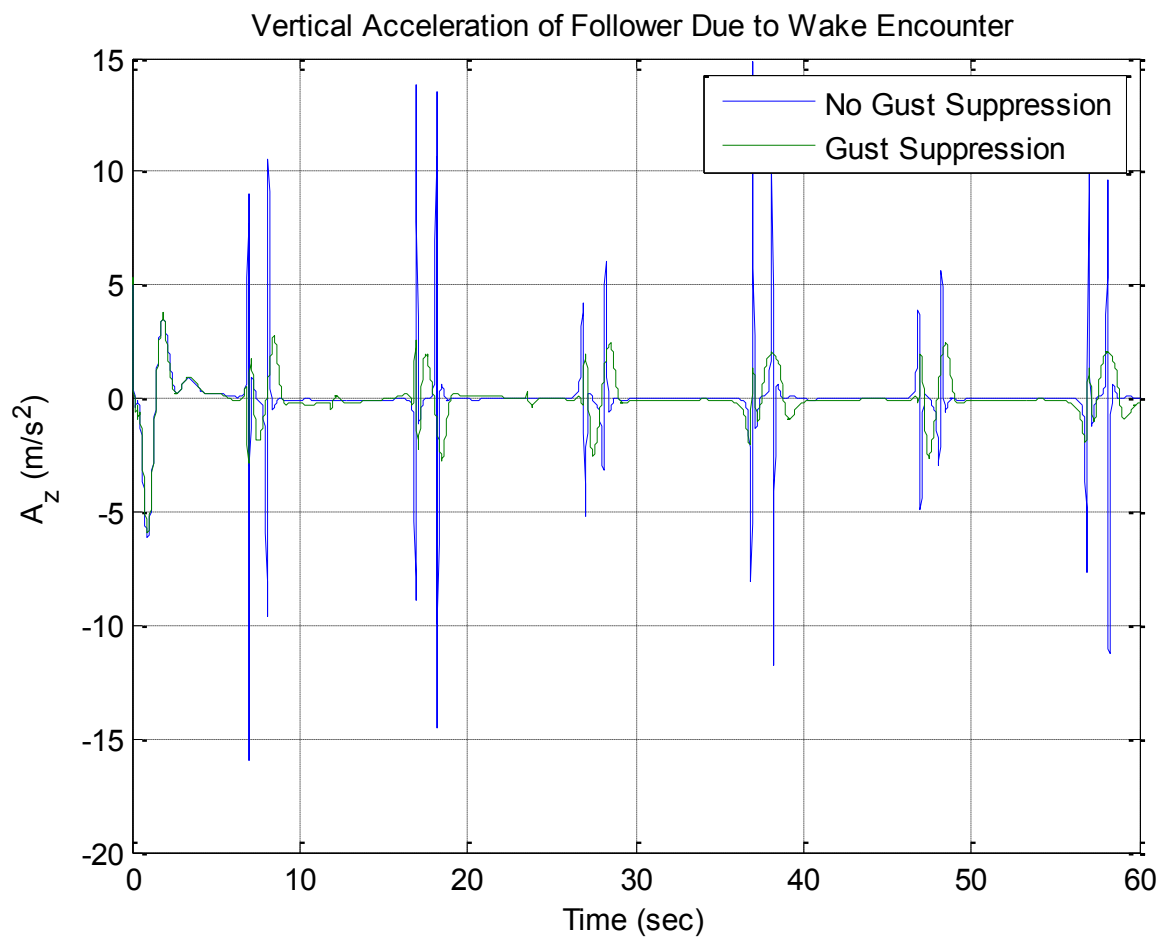

Figure 4.30 Vertical acceleration of the follower UAV as it encounters the leader's wake 


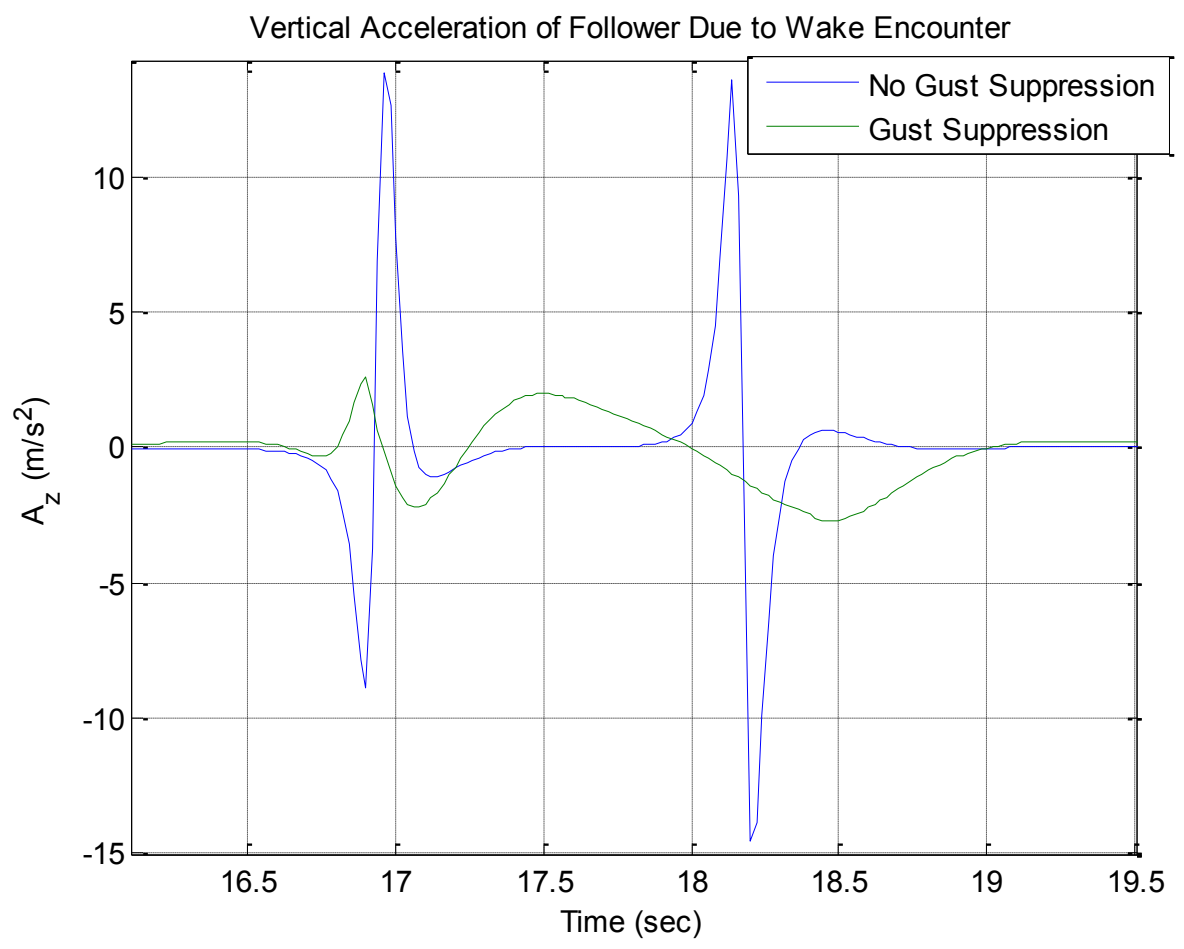

Figure 4.31 Vertical acceleration of the follower UAV as it encounters the leader's wake 


\section{$5 \quad$ Wake Sensing with UAV Formation Flight}

\subsection{Introduction}

Wakes can have a devastating effect on any aircraft by creating a severely turbulent area of rotational flow which can lead to catastrophic accidents. Therefore, the most widely used solution used to deal with them is avoidance which has led to the numerous regulations on how close aircraft can fly as well as how much distance is required between consecutive aircraft takeoffs and landings. Thus, lots of time, money, and energy has gone into the study of wakes so that air traffic can increase while still avoiding hazardous wake conditions. However, through the knowledge of wakes they can also be utilized for energy harvesting.

Multiple wake models have been developed due to the large amount of research into this subject. This chapter starts off discussing these wake models and which one was implemented for simulations shown in this chapter. Next, a discussion on how formation flight was achieved using 2 Phastball research UAVs including the various types of controllers used. After that, wake sensing is shown using flight data gathered from the ADS and IMU. Finally, a few simulations are shown to discuss the potential of implementing cooperative wind sensing where the wind estimation of the follower aircraft receives data from the wind estimation of the leader.

\subsubsection{Wake Models}

Since the study of wing generated wakes has been ongoing for many decades there are a number of wake vortex models already developed. Some of the models frequently used are as follows ${ }^{47}$ :

1. Hallock-Burnham

2. Lamb

3. Combined Rankine

4. Hoffman Joubert

In each of these cases the vortex model calculates a tangential velocity and circulation strength as a function of distance from the core of the vortex. Initializing these models is generally done in the form of calculating a core radius, often a percentage of the lifting surface, and setting the circulation to the theoretical aircraft wake circulation. 
These vortex models are often used because they are computationally simple to implement and they can characterize an entire 2 dimensional flow field by only specifying distance from the core, the core radius, and the circulation parameter. For the research shown here the Hallock-Burnham model was implemented.

\subsection{Modeling}

Wake modeling is important for the verification of wake sensing and can be done using various models but is essentially calculating the circulation strength and core size of the wake and then calculating the effects the wake has on the following aircraft. Figure 5.32 shows the setup for the Phastball research UAV formation flight where each horizontal surface will generate a wake vortex which the follower UAV can detect with its ADS. As an initial result, the method described in this section assumes the induced wind is acting only at the CG of the follower aircraft. In the future the method will be expanded to multiple points about the aircraft which can then be averaged to find a more accurate representation of the effect of induced wind.

The Hallock Burnham vortex ${ }^{48}$ is chosen in this dissertation where the induced wind and any point away from the wake can be calculated using equations (5.1)-(5.3). $\Gamma_{0}$ is the root circulation, $m$ is the mass of the UAV, $g$ is the gravitational constant, $\rho$ is the air density, $s$ and $B$ are the wind area span respectively, $V$ is the velocity of the UAV, and $v_{\theta}$ is the induced wind velocity at a particular distance $R$ from the root core that has a radius of $R_{c}$. Figure 5.33, reproduced from the FAA, shows a sketch of the wake behind an aircraft, which shows two counter-rotating vortexes trailing the wing tips. This sketch shows that disturbances in the atmosphere due to various surfaces on the aircraft are pulled into these two main vortex pairs. 


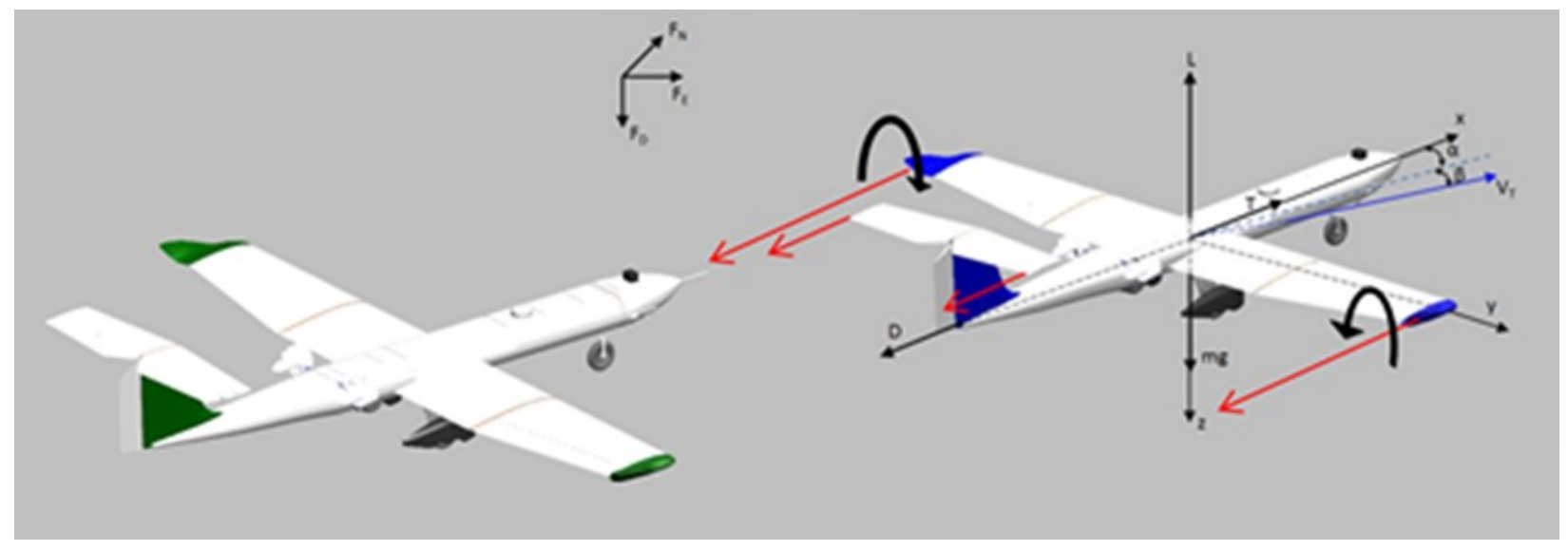

Figure 5.32 Wake modeling for multiple Phastball Research UAVs in formation flight

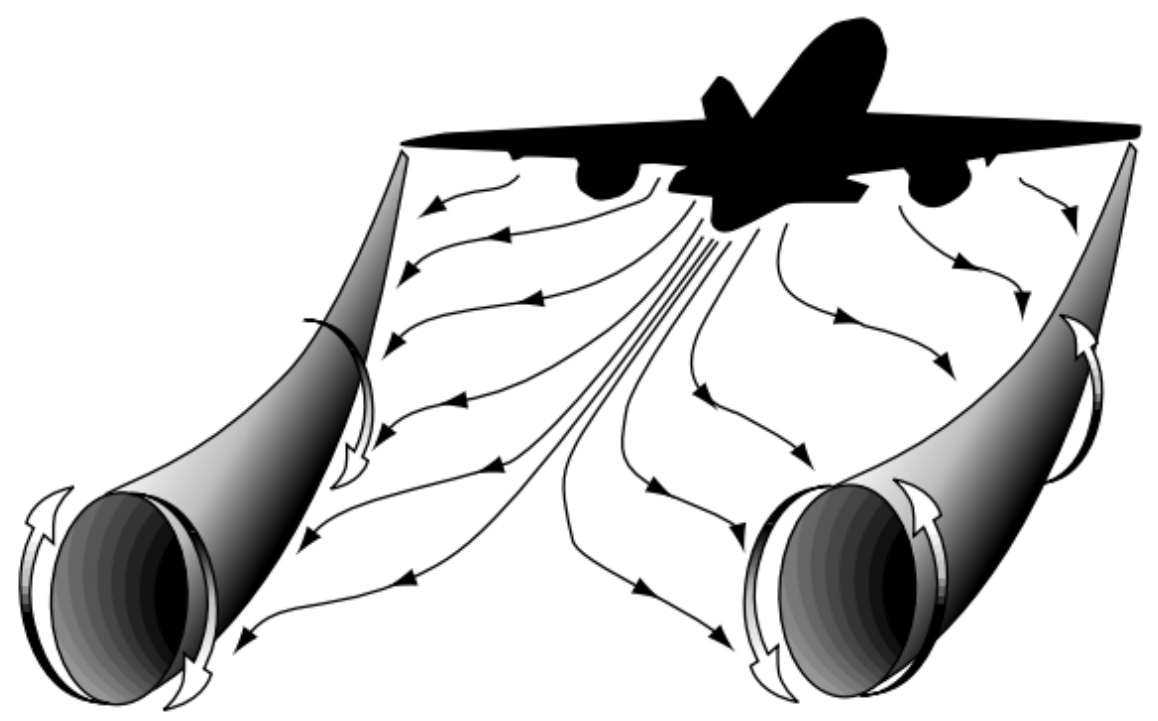

Figure 5.33 Wake model sketch (reproduced from faa.gov)

It is also important to model how the wake moves and decays over time. In order to model these changes equations (5.4) and (5.5) were used in order to capture the descent velocity and strength decay of the wake respectively. Equation (5.4) is used to determine the descent speed of the vortex over time where $\omega_{0}$ is the descent speed and $b_{0}$ is $1 / 4$ of pi times the wing span of the UAV. Equation (5.5) shows the decay of the vortex as a function of distance $x$ from where the wake was generated. Furthermore $\tau$ is determined by the turbulence level in the atmosphere, assumed here to be light turbulence since flight testing is generally conducted in 
calm weather conditions. Finally, the induced velocity can be converted to the inertial frame using equation (5.6) where $\left[V_{y}, V_{z}\right]^{T}$ is the velocity along the $y$ and $z$ axis in the body frame induced on the follower from the leader's wake and $\varphi$ is the angle that the follower is rotated about the wake of the leader. Constants in each of these equations are described below in Table 5.9.

$$
\begin{aligned}
& \Gamma_{0}=\frac{m g}{\rho s B V} \\
& R_{c}=0.05 B \\
& v_{\theta}(r)=\frac{\Gamma_{0}}{2 \pi R} \frac{R^{2}}{R^{2}+R_{c}^{2}} \\
& \omega_{0}=\frac{\Gamma_{0}}{2 \pi b_{0}} \\
& \Gamma(x)=\frac{\Gamma_{0} e^{\left(-0.45 x \Gamma_{0}\right)^{0.25}}}{\rho V B} \\
& {\left[\begin{array}{l}
V_{y} \\
V_{z}
\end{array}\right]=\left[\begin{array}{c}
-v_{\theta} \sin (\varphi) \\
v_{\theta} \cos (\varphi)
\end{array}\right]}
\end{aligned}
$$


Table 5.9 Constants used in wake modeling

\begin{tabular}{llll}
\hline Variable & Description & Value & Units \\
\hline$R_{c}$ & Root Core & 0.0912 & $\mathrm{~m}$ \\
$\Gamma_{0}$ & Root Circulation & 1.72 & $\mathrm{~m}^{2} / \mathrm{s}$ \\
$B$ & Wing Span & 2.4 & $\mathrm{~m}$ \\
$S$ & Wing Area & 0.73 & $\mathrm{~m}^{2}$ \\
$m$ & Mass & 11 & $\mathrm{~kg}$ \\
$g$ & Gravitational Constant & 9.81 & $\mathrm{~m} / \mathrm{s}$ \\
$\rho$ & Air Density & 1.2727 & $\mathrm{~kg} / \mathrm{m}^{3}$ \\
$\omega_{0}$ & Descent Velocity & 0.156 & $\mathrm{~m} / \mathrm{s}$ \\
$\tau$ & Light Turbulence Level & 0.003 & $\mathrm{~N} / \mathrm{A}$ \\
\hline
\end{tabular}

Figure 5.34 Induced vertical velocity from the wake of the Phastball Research UAV below shows the induced vertical velocity form the Phastball research UAV using equations (5.1) through (5.6). Only vertical velocity is shown here as it is important for energy harvesting when flying in formation. As described above inboard of the wing tips the induced wind maxes out at just over $-1.6 \mathrm{~m} / \mathrm{s}$ while outboard of the wings the induced wind reaches almost $1.4 \mathrm{~m} / \mathrm{s}$.

Finally, it is important to note two important key aspects of the wake. First, the wake will roll up in the near field (5-15 wingspans), followed by a stable vortex pair in the mid field (15 to 150 wingspans) ${ }^{19}$. For applications with small UAVs it is assumed that the wake will maintain its core radius and strength for locations between 5 to 30 wingspans. This is due to the slow descending velocity and the flow evolution of wake vortices generated by small UAVs ${ }^{49}$. Secondly, the wake will flow parallel to the aircraft velocity vector which will affect the position of the wake ${ }^{15}$. 


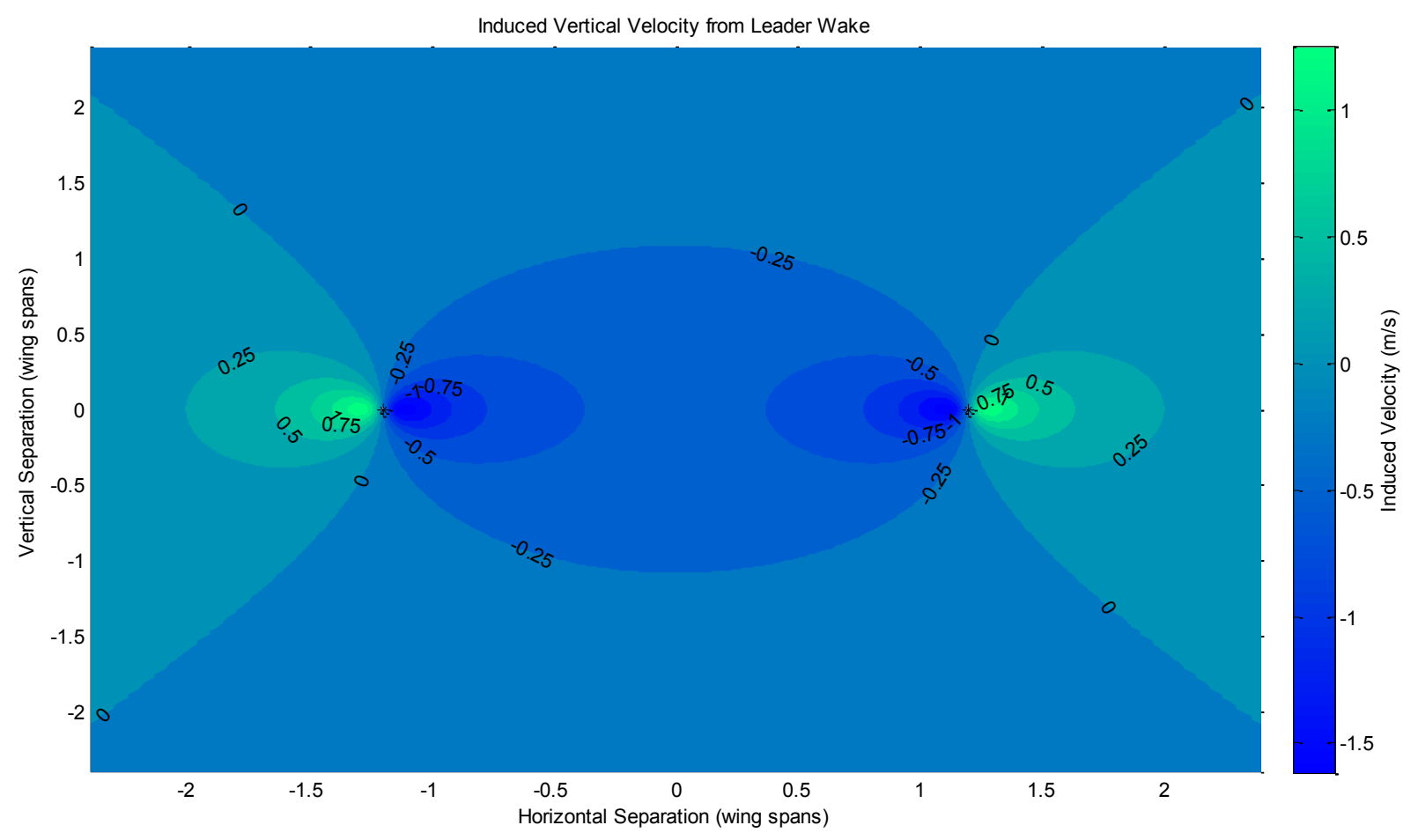

Figure 5.34 Induced vertical velocity from the wake of the Phastball Research UAV

\subsection{Phastball Research UAV Formation Flight Setup}

For wake sensing the Phastball research UAVs were flown in formation, shown below in Figure 5.35 , to collect data. In order to perform a formation flight a leader-follower formation was adopted where one pilot controls the leader aircraft and the follower executes the formation control laws in order to maintain a desired position and orientation with respect to the leader aircraft. This is accomplished through the use of an Inner Loop Controller (ILC) and an Outer Loop Controller (OLC). The OLC takes in the states of both the leader aircraft and itself in order to calculate a desired attitude for path following as well as a desired throttle setting. This desired attitude is fed into the ILC which sends commands to the control surfaces to maintain the desired attitude.

The ILC was implemented first and was tested by commanding a desired roll and pitch and looking at the steady state error in desired versus actual attitude. The desired attitude was offset by two degrees in the controller since the Phasball research UAV trims at two degrees of 
pitch under nominal conditions. After each test flight of the ILC the gains were tuned until desired controller performance was achieved. In order to implement the OLC a virtual leader was developed to reduce the risk of multiple UAVs in the airspace at the same time. The virtual leader updates itself to always start at a preset distance in front of the UAV. This ensured that the flight controller would not try any sudden or abrupt maneuvers in order to obtain the correct formation at the beginning of formation flight. Similar to the ILC the OLC was tuned after each flight with the virtual leader until satisfactory controller performance was achieved through a complete flight lap.

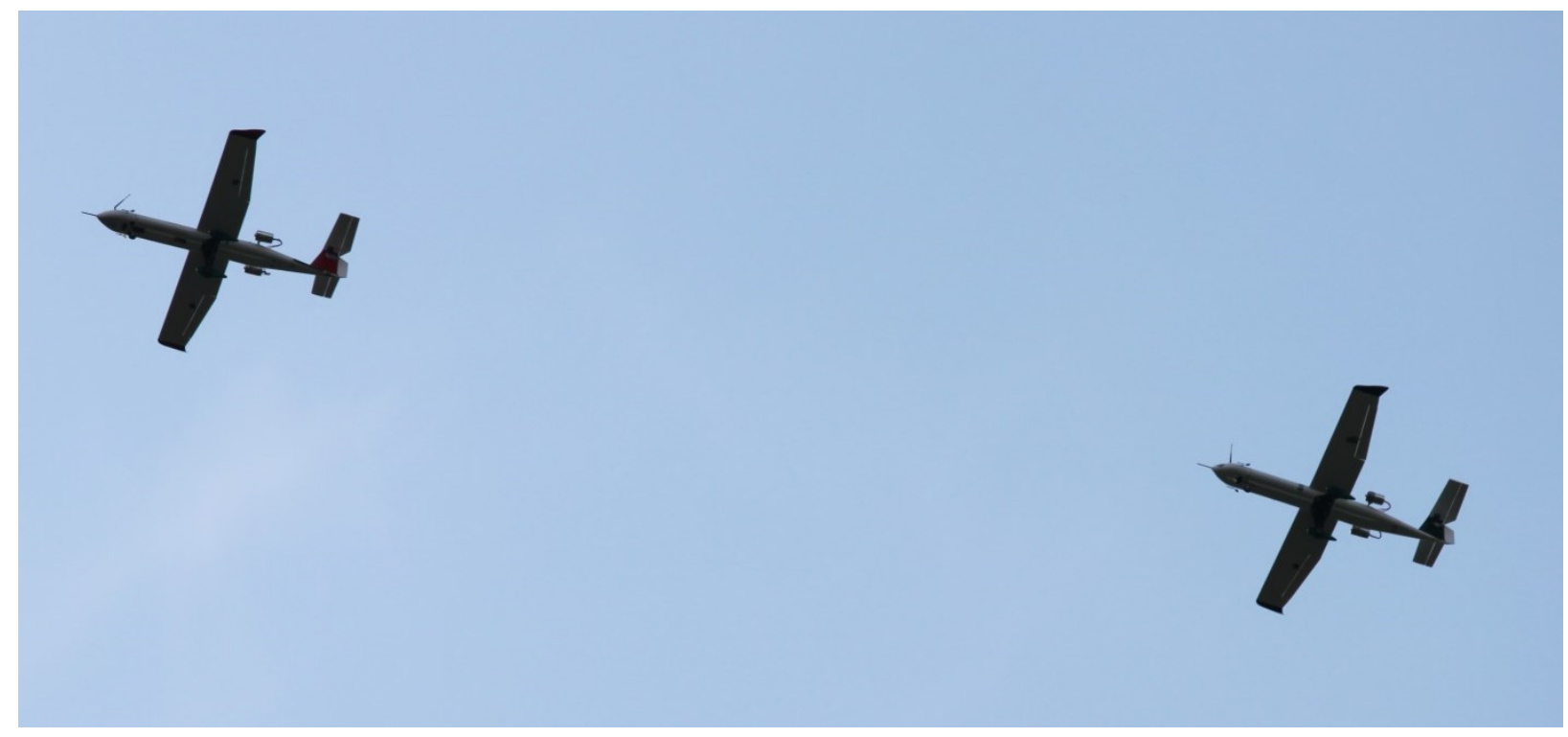

Figure 5.35 Phastball Research UAV formation flight

\subsubsection{Flight Controller Development}

\subsubsection{Virtual Leader Development}

During flight testing it was important to ensure that the controller worked properly before performing flights in formation. In order to accomplish this numerous flight with a virtual leader were conducted where the flight computer was fed virtual position and velocity data as if there was a leader even though there wasn't. The virtual leader always started at a predefine position in front of the follower and flew a perfect lap from that point. This had a few advantages when evaluating the flight controller. First, it made analyzing the controller error easier since the 
leader flew a perfect flight path. The virtual leader also allowed for a single pilot for flight testing since only one plane was in the air at a time. It also drastically reduced potential dangers of flying formation flight without being able to verify controller performance.

\subsubsection{Outerloop Control}

The outer loop controller was designed as two decoupled controllers, one for the vertical formation geometry and another for the horizontal formation geometry ${ }^{50}$. The vertical controller is a linear altitude tracker which calculates a desired pitch angle required for the follower UAV to match the altitude of the leader's altitude. The horizontal controller, however, calculates the desired throttle to maintain the commanded forward separation and a desired roll angle to maintain a commanded lateral separation. This is all done through Non-Linear Dynamic Inversion (NLDI) which converts a nonlinear system into a linear one so that linear control techniques can be applied. The OLC uses the equations detailed in [50] but reworked for use with the tracking problem. The main responsibility of the OLC is to calculate the errors between the follower's position and its desired position and to then calculate reference angles to minimize these errors. The OLC also calculates a throttle setting based on the forward error between the leader and the follower.

\subsubsection{Innerloop Control}

In order to track the leader the follower UAV must be able to calculate the appropriate surface deflection commands in order to maintain the desired attitude developed within the OLC, this is done within the ILC ${ }^{51}$. The ILC was designed using Linear Quadratic (LQ) techniques which begin with the development of a linear aircraft model described in [52]. The LQ control laws were developed using the methods in [53]. This development of the ILC implies independence between the longitudinal and lateral dynamics of the UAV and therefore can be separated into two controllers. The following are inputs to the longitudinal and lateral ILCs:

$$
\begin{aligned}
& X_{\text {lat }}=[\beta, p, r, \phi] \\
& X_{\text {long }}=[\alpha, q, \theta]
\end{aligned}
$$


where $X$ denotes the inputs consisting of $[\beta, p, r, \phi]$ which are the measured side slip angle, roll and yaw rates, and roll angel respectively and $[\alpha, q, \theta]$ which are the measured angle of attack, pitch rate, and pitch angle respectively. From these inputs the ILC creates the following surface control deflections:

$$
\begin{gathered}
U_{\text {lat }}=\left[\begin{array}{l}
\delta_{\text {ail }} \\
\delta_{\text {rud }}
\end{array}\right] \\
U_{\text {lat }}=\left[\delta_{\text {elev }}\right]
\end{gathered}
$$

where $U$ denotes the output of the controller and $\left[\delta_{\text {ail }}, \delta_{\text {rud }}, \delta_{\text {elev }}\right]$ are the deflections in degrees of the aileron, rudder, and elevator control surfaces respectively.

\subsubsection{Variable Separation Control}

It is important to get as much data during each flight as possible because of the limited amount of flight time and flight days that are conduced each year. Because of this the OLC was modified in order to take a constant commanded separation as well as inputs from the pilot so that multiple areas behind the leader could be explored to get a better understanding of the leader's wake. Since the characteristics of the wake are often defined in units of wingspans behind the aircraft, the constant offset as well as the pilot commands range is also in units of wingspans. As discussed above the wing span for the Phastball research UAV is approximately

\section{4 meters.}

The pilot's command range was divided into five equal segments and the pilot could command any of those five preset separation distances. The characteristics of the variable separation control can be seen in Table 5.10. The constant offset of the OLC was set to fly directly behind the leader's wingtip at a distance of ten wingspans. This was decided as the base separation amount because directly behind the UAV's wingtip from five to fifteen wingspans is where the interesting characteristics of the wake would be found ${ }^{19}$. It is important to note here that the pilot commands the desired separation using normal flight channels, throttle, aileron, and elevator for forward, lateral, and vertical separations respectively. Both 
the aileron and elevator channels commanded a desired separation that is the same as normal flight, e.g., elevator down moves the follower above the leader and right aileron moves the follower to the right of the leader. The throttle, however, is reversed so that an increase in throttle moves the follower further behind the leader and vice versa. The reason for this is safety, if the follower and leader are in close formation and the pilot needs to disengage the follower's throttle cuts to a minimum and the aircraft will not collide with the leader.

\section{Table 5.10 Variable separation control characteristics}

\begin{tabular}{llll}
\hline Separation Axis & Forward & Lateral & Vertical \\
\hline Constant Offset & 10 & 1.2 & 0 \\
Pilots Command Range & $\pm 5(5: 15)$ & $\pm 10(12.4:-7.6)$ & $\pm 5(-5: 5)$ \\
Pilots Control Input & Throttle & Aileron & Elevator \\
\hline
\end{tabular}

\subsection{Wake Sensing Through Flight Tests}

There have been multiple studies into the wakes of aircraft before this research dating back many decades but all have been done utilizing two manned aircraft flying in formation where both aircraft are under manual control. The first part of this section will show wake visualization using a small UAV and a smoke screen. The flight results in the rest of this chapter are some of the first results of wake sensing using multiple UAVs flying in formation where the follower is flying autonomously.

\subsubsection{Wake Visualization Using a Small UAV}

Wake visualization is a pretty straight forward process in order to physically see the wake behind a small UAV. During a flight test day smoke bombs were set off and a small UAV with an attached camera was flown through the smoke. Figure 5.36 shows the wake produced as the UAV flew through the edge of the smoke cloud and shown zoomed in in Figure 5.37. A few things can be seen from these images, first is that the center of each vortex does not have any smoke which shows that the influence of the vortex goes to zero at the core of the wake. Next, it is important to note that through video data it can be shown that the wake stays spinning for quite some time without losing much altitude which verifies small descent speed used in the modeling of the wake. Finally, it is good to note the size of the vortices and how there are two 
well defined counter rotating vortices behind the UAV. They are not very large, on the order of about a third of the wingspan and do not seem to effect each other. In fact it seems that the air directly behind the aircraft is barely, if at all affected by the wing's wake.

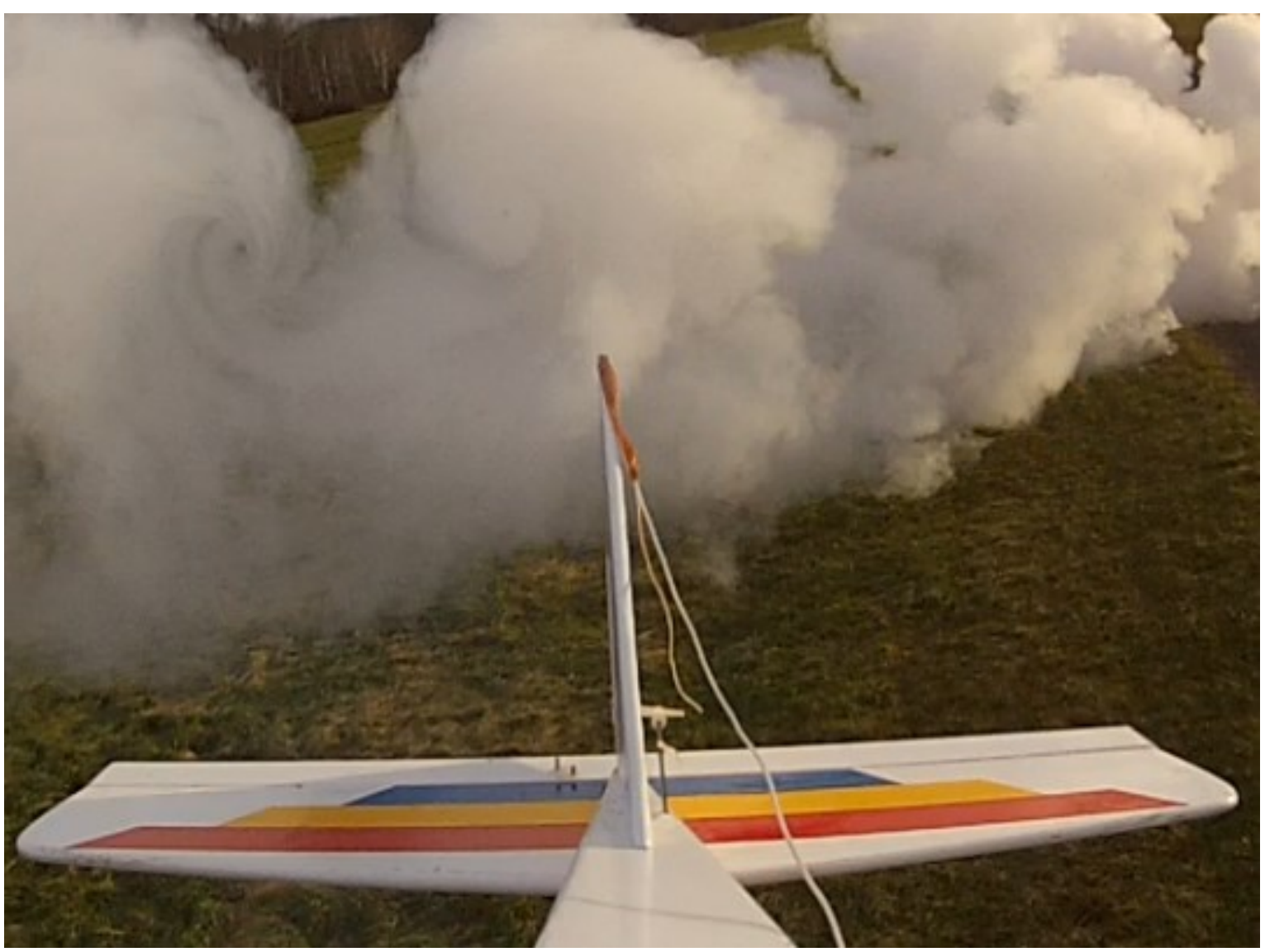

Figure 5.36 Wake visualization using smoke and small UAV 


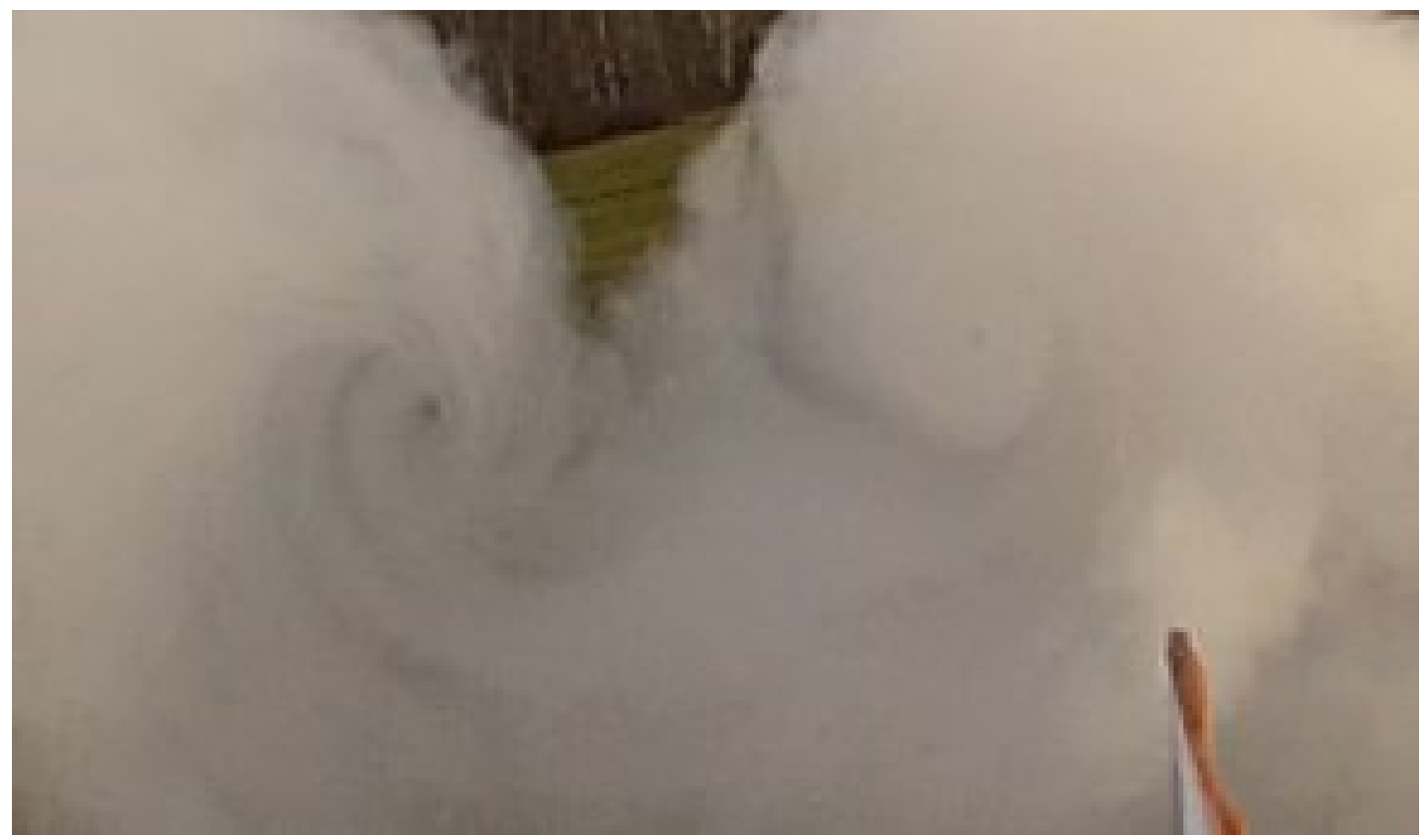

Figure 5.37 Wake visualization using smoke and small UAV (detailed)

\subsubsection{Wake Sensing During Formation Flight}

As mentioned flight testing was conducted with two Phastball research UAVs in formation flight in order to perform wake sensing. The results of which are shown in Figure 5.38 through Figure 5.43 below consists of flying with the follower's nose directly behind the wing tip of the leader, or in other words, with a clearance of 5 wingspans in the forward direction, 0 wingspans in the vertical direction, and $1 / 2$ a wingspan in the lateral direction.

First, it is important to note that the UAV is being controlled to follower the aircraft of the leader by a preset values as discussed in section 5.3.1. Because of this as the wake effects the measurements of the AoA vanes as well as the pitch and pitch rate of the follower the controller will be effected since these are the inputs to the longitudinal controller. Therefore, the encounters with the wake tend to be short as the UAV will try to maintain certain flight conditions using sensors that are affected by the wake. It is also good to realize that often the wake is only sensed by one AoA vane. This is because the generated wake is relatively small, about the same size as the diameter of the nose cone, so having both vanes encounter different parts of the wake simultaneously is a very difficult task. 
For instance, suppose the follower is following the left wing tip of the leader and therefore is flying right in the center of the generated vortex. The follower's left vane will sense an increase in AoA, since the vane will tend to point downward into the wake's upwash region while the right vane will sense a decrease in AoA since the vane will tend to point upward into the wake's downwash region. Because of this the plots below show the AoA according to the left and right flow vanes as well as the difference between the two. Under steady level flight and in the absence of wake effects or variable wind conditions the difference between the two vanes should be zero.

As the follower flies through the leader's wake the generated downwash and upwash will be sensed by the airflow vanes on the left and right side of the Phastball research UAV, the accelerometer will sense the aircraft being pushed around by the induced wind, and the pitch angle will change as the follower is in a region of upwash or downwash. Again since the ILC will fight the effects of the wake on the follower the wakes effect on the UAV will not be as pronounced as it would be if the UAV where allowed to maintain flight within the wake.

Figure 5.38 and Figure 5.39 show the vane data from a virtual leader flight and a formation flight respectively. What is important to notice here is that during virtual leader flights the difference between the left and right AoA vanes is nearly zero for the entire flight. Next, the data shown during formation flight has spikes in the difference between the two vanes regularly during the straight leg of the flights. 
Follower Aircraft Data

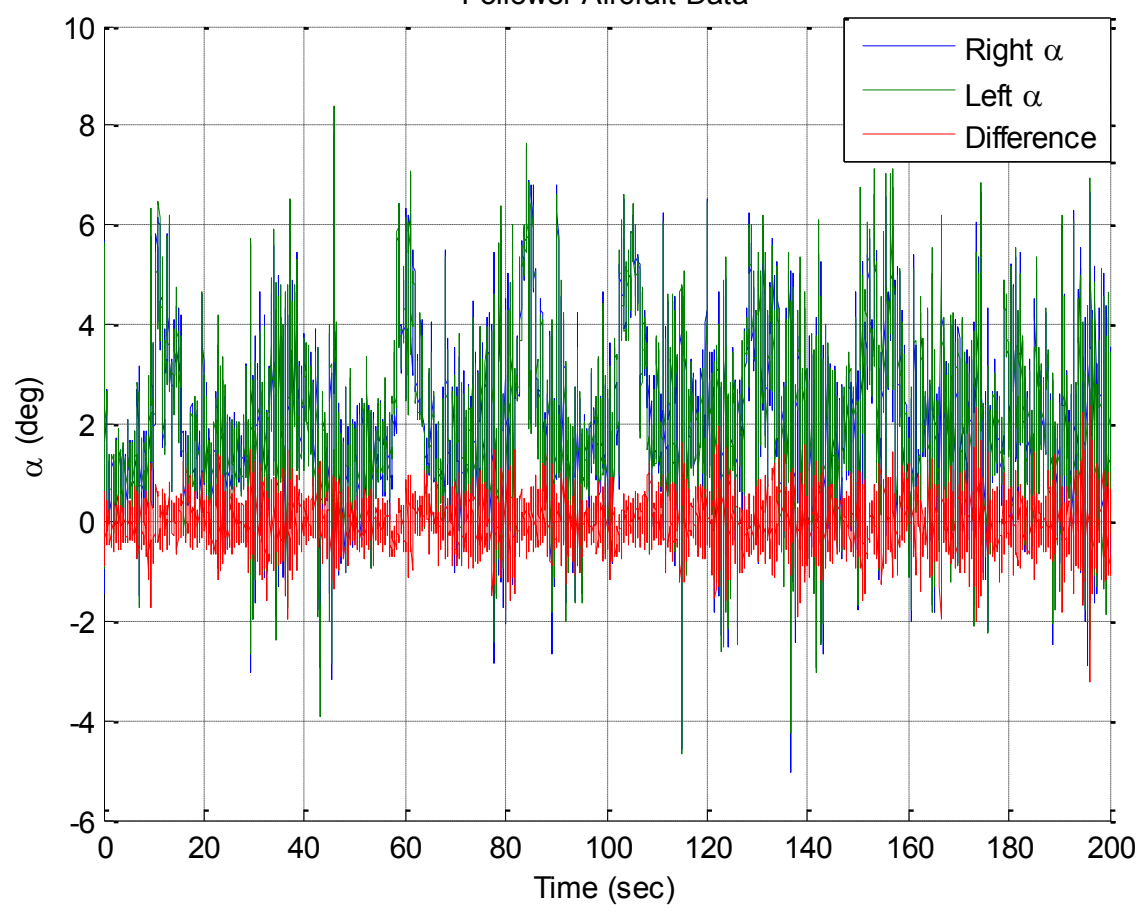

Figure 5.38 Follower aircraft data during normal flight

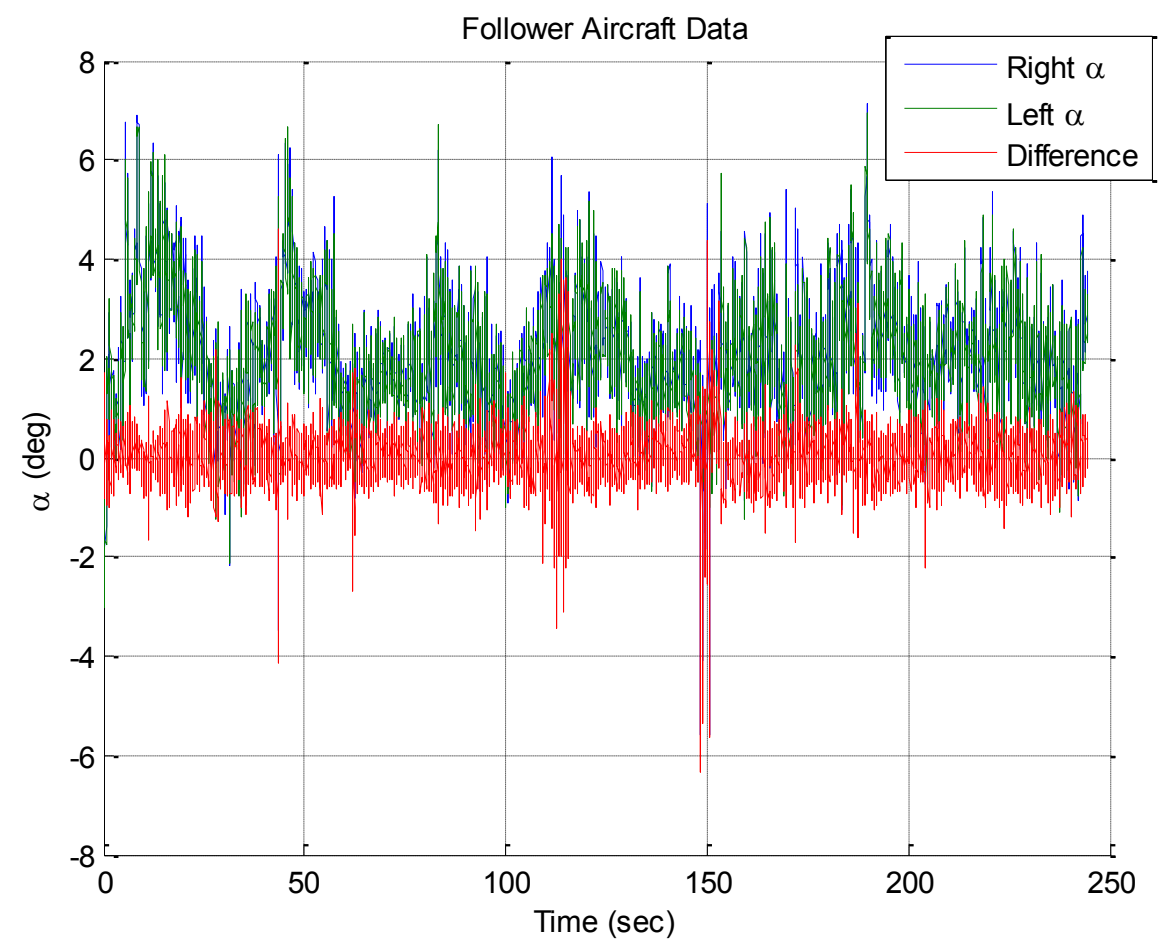

Figure 5.39 Follower flight data during formation flight 
Figure 5.40 shows an encounter with the wake that lasts for nearly four seconds with both AoA vanes getting pushed around quite a bit. This is the longest duration of flight where the follower is sensing the leader's wake the entire time and the follower is moving from inboard to outboard as compared the leader. This is seen in the data where at the beginning of the encounter the left vane experiences a small decrease in AoA while the right vane is experiencing an increase in AoA which is expected if the follower's nose is directly behind the leader's wing tip. As the follower moves outboard a bit due to imperfections in the controller and atmospheric turbulences the left vane hardly picks up the wake while the right vane senses a large increase in AoA as it now encounters the upwash of the leader's wake.

In this case the aircraft is bouncing around through the wake and so it is difficult to see the effects of this wake encounter in the accelerometer data. However, it is still noticeable in the aircraft pitch angle which shows the aircraft with a negative induced pitch each time the UAV experiences a prolonged exposure to the downwash of the leader.

Figure 5.41 and Figure 5.43 show much more abrupt encounters between the follower UAV and the leader's wake, which is how most of the wake encounters in the flight data look. Figure 5.41 clearly shows the right vane encounter the downwash of the wake and then quickly hit the upwash of the leader's wake. Approximately half a second after the vanes sense the wake of the leader the dynamics show the encounter with the wake as well. The vanes showed the aircraft hitting the downwash of the wake followed by the upwash which produced a negative pitch in the aircraft of about 5 degrees which than leveled off. This is also shown in the accelerometer data where the UAV goes from experiencing an acceleration of $4 \mathrm{~m} / \mathrm{s}^{2}$ to nearly $5 \mathrm{~m} / \mathrm{s}^{2}$ which is a change of almost $1 \mathrm{~g}$ in about a second and a half of flight. This type of change in acceleration could easily be felt by passengers on an aircraft and would not be very comfortable to experience.

Figure 5.42 shows a similar wake encounter where in this case the left AoA vane encounters the upwash of the leader's wake recording an AoA of over 9 degrees. The interesting difference in this encounter is that there is a much longer residual effect of the wake on the AoA vane as it takes nearly half a second for the vane to return to similar readings of the right vane. In this 
case the upwash causes a slight increase in the pitch of the aircraft starting again about a half second after the vane initially senses the wake. Most likely due to the short duration of the encounter and that the rest of the UAV is under effects from the wake and atmosphere there is not much wake evidence shown in the accelerometer data.

Figure 5.43 shows flight data where the right AoA vane quickly senses part of the downwash of the wake but the effects are so weak that the encounter is not noticeable in the pitch or accelerometer data of the follower. About two seconds later though the left AoA vane encounters the upwash of the leader's wake which can clearly be seen in the AoA reading of nearly 13 degrees and a difference in vane readings of about 9 degrees. This data also clearly shows an induced pitch from the encounter of nearly 8 degrees and a rather sudden decrease in vertical acceleration by about $7 \mathrm{~m} / \mathrm{s}^{2}$ within a quarter of a second followed by an increase in vertical acceleration by nearly $9 \mathrm{~m} / \mathrm{s}^{2}$ over about second of flight. This type of abrupt changes in acceleration would be clearly felt by passengers and could lead to both aircraft fatigue and personal injuries. 
Follower Aircraft Data
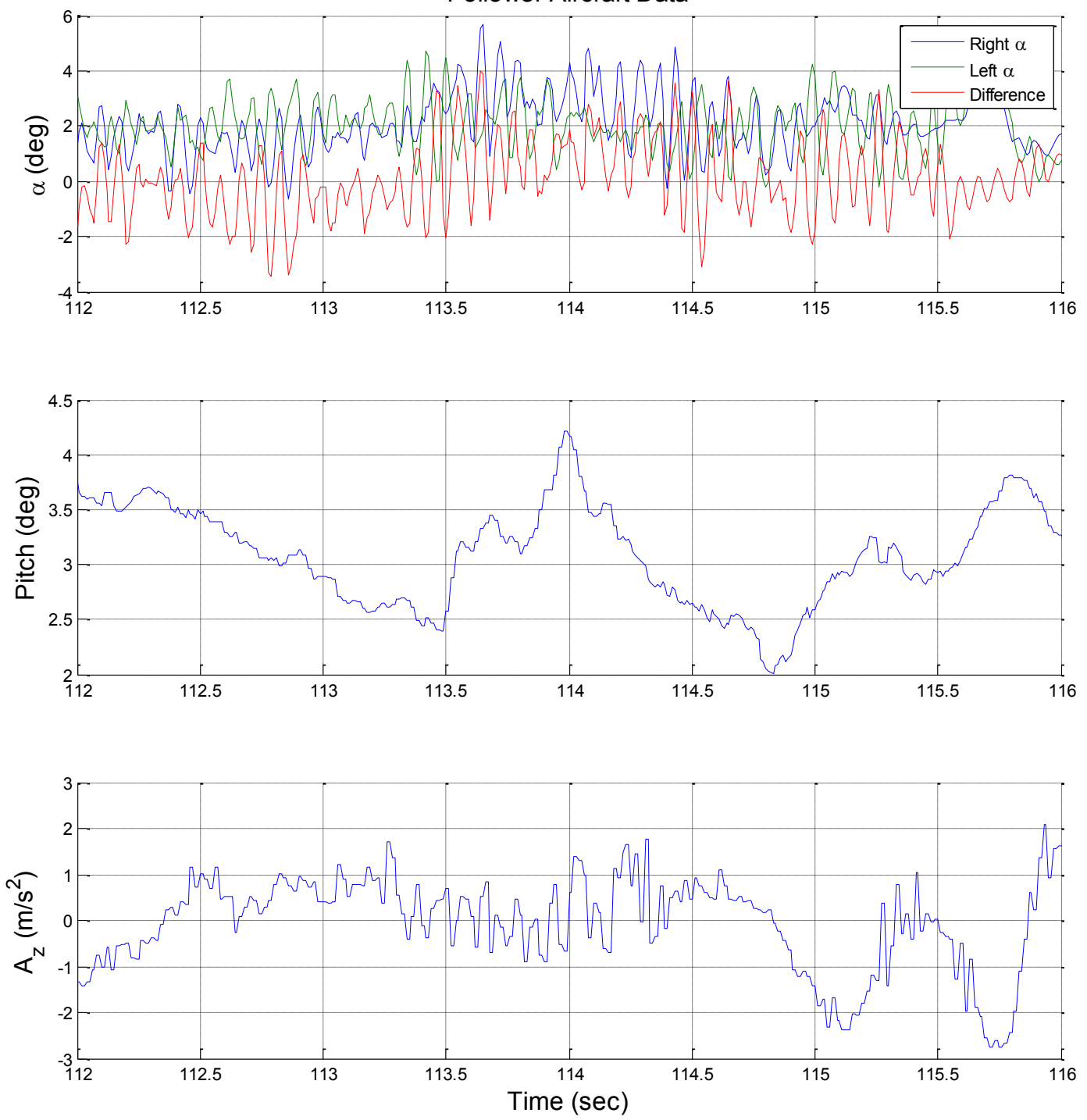

Figure 5.40 Follower wake sensing data during formation flight 

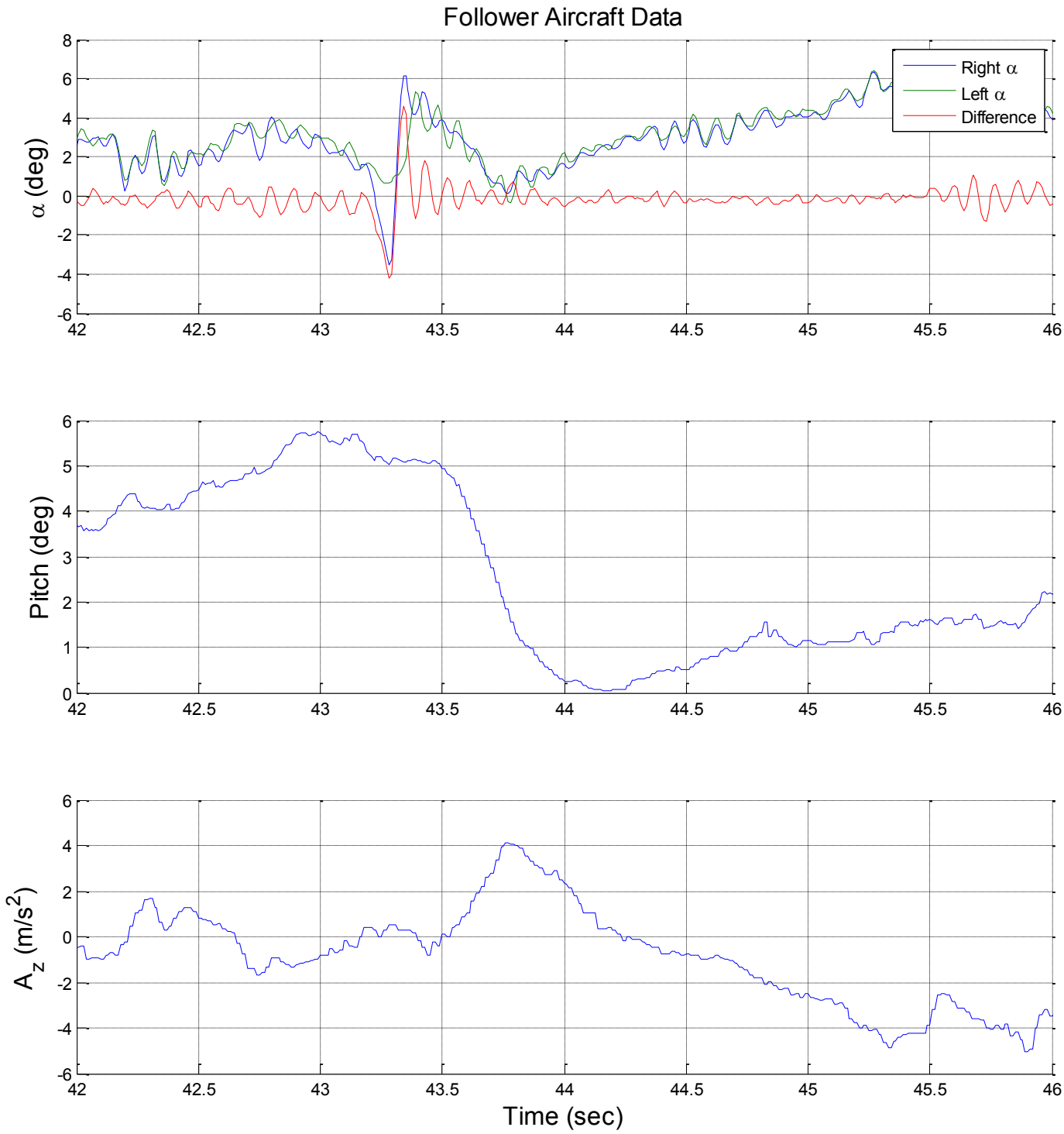

Figure 5.41 Follower wake sensing data during formation flight 

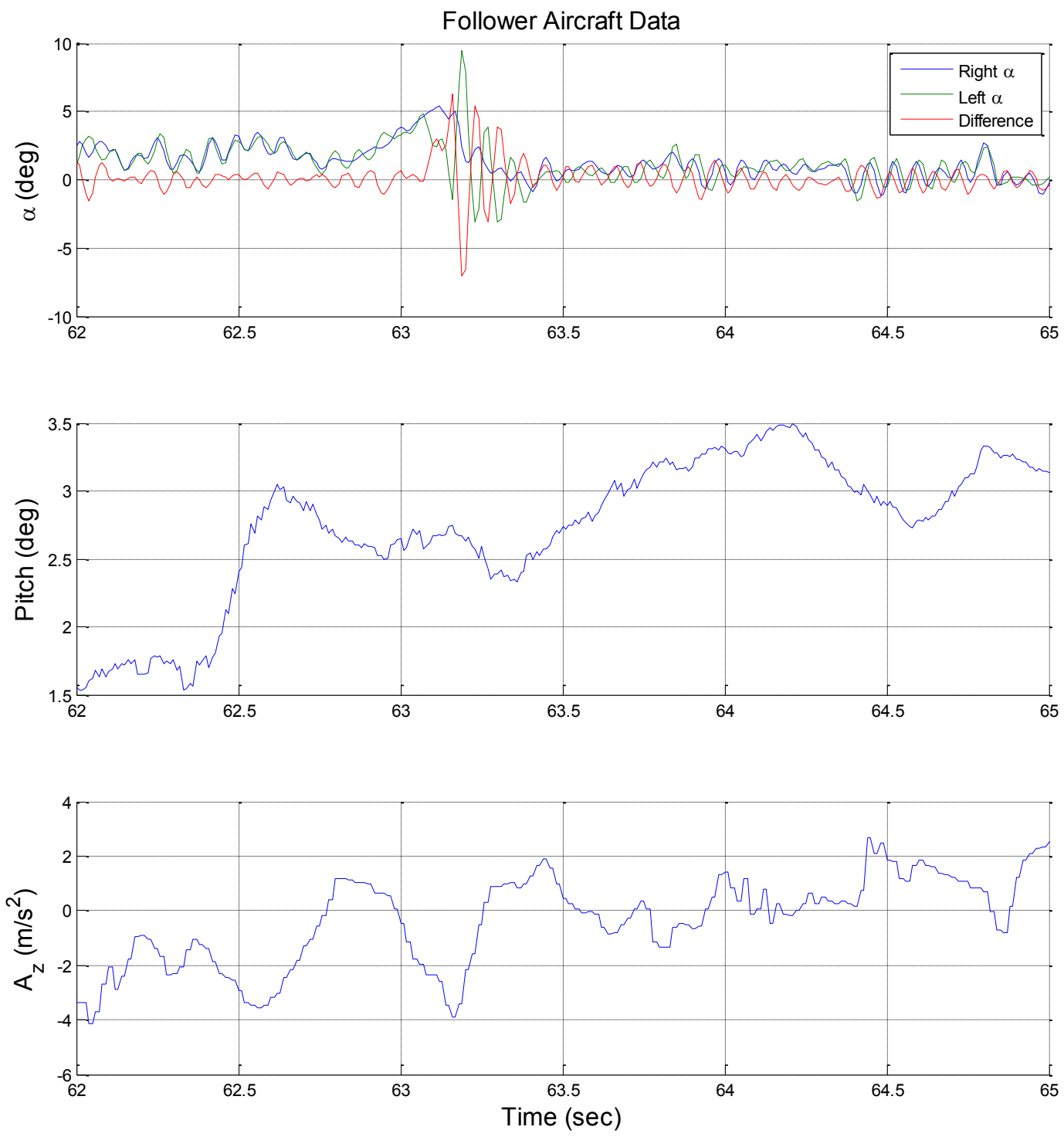

Figure 5.42 Follower sensor data during wake sensing 

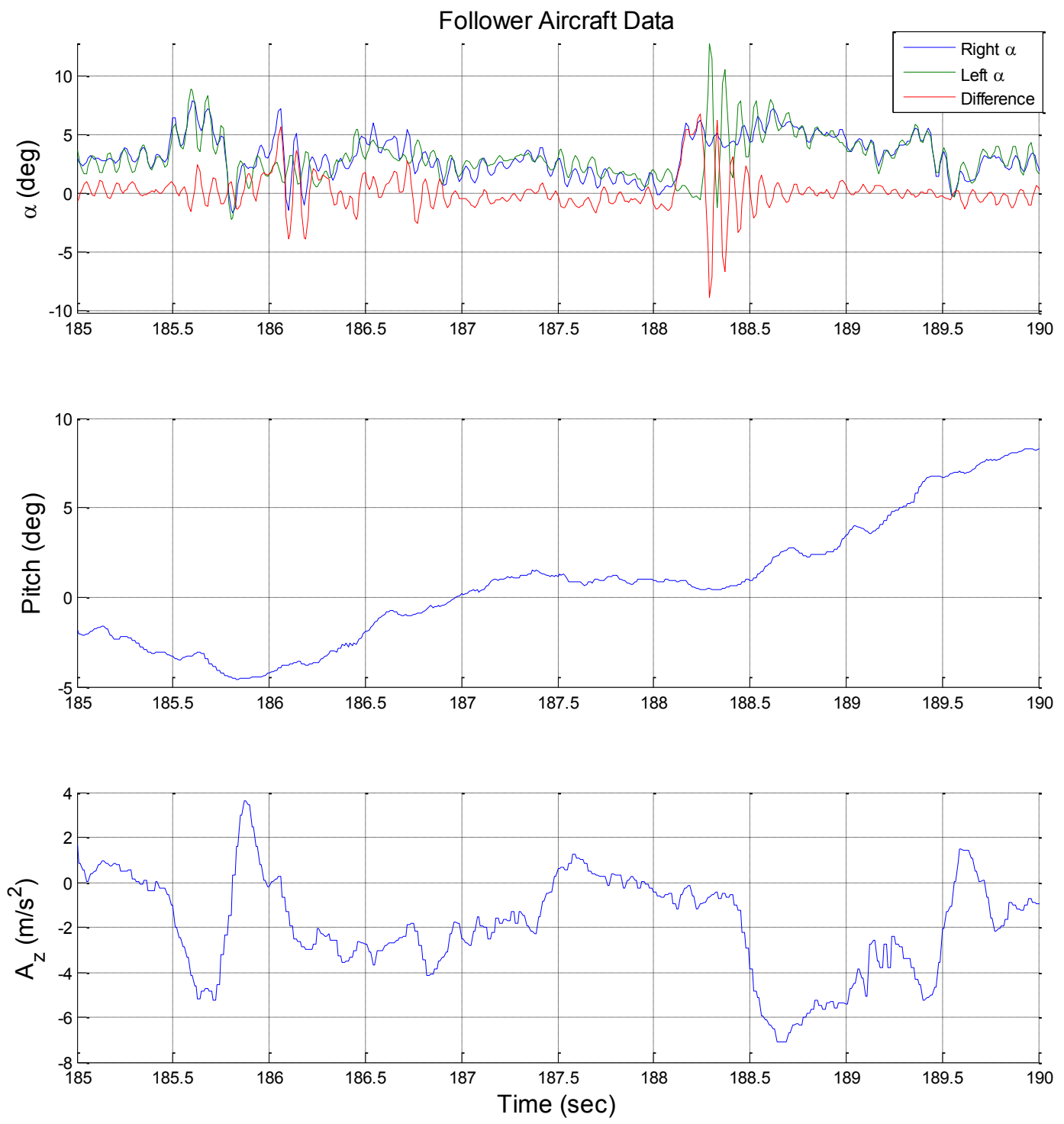

Figure 5.43 Follower wake sensing data during formation flight 


\subsection{Further Topics}

\subsubsection{Sweet Spot for Maximum Energy Savings}

In formation flight there is a point behind the leader that is often referred to as the sweet spot $^{16}$ where the follower gets the most benefit from the leader's wake. The sweet spot is in the upwash from the leader's wake which reduces the drag of the follower and therefore allows the follower to use less energy for flight. Because of the physics of the wake and how it is generated the area of upwash is found just outside of the wing tips. It has been shown that flying within the sweet spot of the leader's wake can have a fuel savings of greater than 18 percent $^{17,18}$.

This area was looked into towards the end of this project and it was found that for most of the cases shown there was little evidence to suggest much fuel savings during formation flight testing. The least amount of power used was calculated on a flight where the follower was at a lateral separation of 1 wingspan which is what most literature suggests the sweet spot would be. This one case is hardly enough to make any conclusions and future investigation into flying within the sweet spot using small UAVs is a topic of great interest for the FCSL.

\subsubsection{Cooperative Wind Sensing}

For cooperative wind sensing the UKF described above (section 4.4) was converted for the follower UAV in order to include the wind estimation of the leader as a measurement. This was accomplished by modifying equation (4.12) and adding in the estimated wind states of the leader aircraft, $\left[\hat{w}_{L N}, \hat{w}_{L E}, \hat{w}_{L D}\right]$, in the inertial frame shown in equation (5.11). Finally, the wake modeling described in section 5.2 was used to determine the induced wind on the follower from the leader's wake. Table 5.11 below shows the advantages of using cooperative wind gust estimation where the mean of the error is reduced significantly from using follower flight data only.

$$
\left[\begin{array}{l}
V_{x} \\
V_{y} \\
V_{z}
\end{array}\right]=\operatorname{DCM}(\phi, \theta, \psi)\left[\begin{array}{c}
u \\
v \\
w
\end{array}\right]+\left[\begin{array}{c}
W_{N} \\
W_{E} \\
W_{D}
\end{array}\right]+\left[\begin{array}{c}
\hat{W}_{L N} \\
\hat{W}_{L E} \\
\hat{W}_{L D}
\end{array}\right]
$$


Figure 5.44 and Figure 5.45 show the wind fields used for the simulation for cooperative wind sensing for the follower and leader UAV respectively. Since the wind is modeled as varying spatially the wind field is slightly delayed between the leader and the follower. As shown there was a sinusoidal wind field along the North and East axis with a magnitude of 2.5 and $1 \mathrm{~m} / \mathrm{s}$ respectively with a bias of 3 and $2 \mathrm{~m} / \mathrm{s}$ respectively. There is a $1 \mathrm{~m} / \mathrm{s}$ constant wind along the Down axis for both UAVs.

Figure 5.47 shows the UKF estimation of wind using only leader data; this was done as a base line in order to compare estimation under various scenarios. The UKF estimates wind in the North and East directions fairly well while estimating the wind in the Down direction not as well. The main reason for this is that the aircraft is flying with steady altitude and therefore does not have a lot of information about the wind along the Down axis. Figure 5.48 shows the UKF estimation of wind using only follower data. It is a bit more chaotic as gusts are added to the estimation and it does a poor job of estimating the wind along the East axis.

Figure 5.49, on the other hand, shows the estimation of the same wind field and induced wind using cooperative techniques discussed above. The maximum error is at most $1 / 2 \mathrm{~m} / \mathrm{s}$ off of the truth while generally it is very close to the actual values. Figure 5.50 and Figure 5.51 show the individual estimated wind states against truth data. It is clear here that even though the filter does slightly worse in estimating the wind along the North axis for cooperative wind estimation it does a much better job along the East axis and moderately better along the Down axis. Basically, the wind estimation along the East axis of the leader keeps the estimation of the follower from diverging to far from the wind field.

In essence because of the increase in turbulence and induced wind gusts the UKF estimation of the wind states sensed by the follower are never as good as the leader. However, by using the wind estimation of the leader the follower can estimate the wind with much greater precision. Table 5.11 shows the statistics of UKF wind estimation using leader data only, follower data only, and cooperative wind estimation. Using cooperative wind estimation the mean of the error over all three axis dropped by over $72 \%$ and the standard deviation improved by over $78 \%$. 


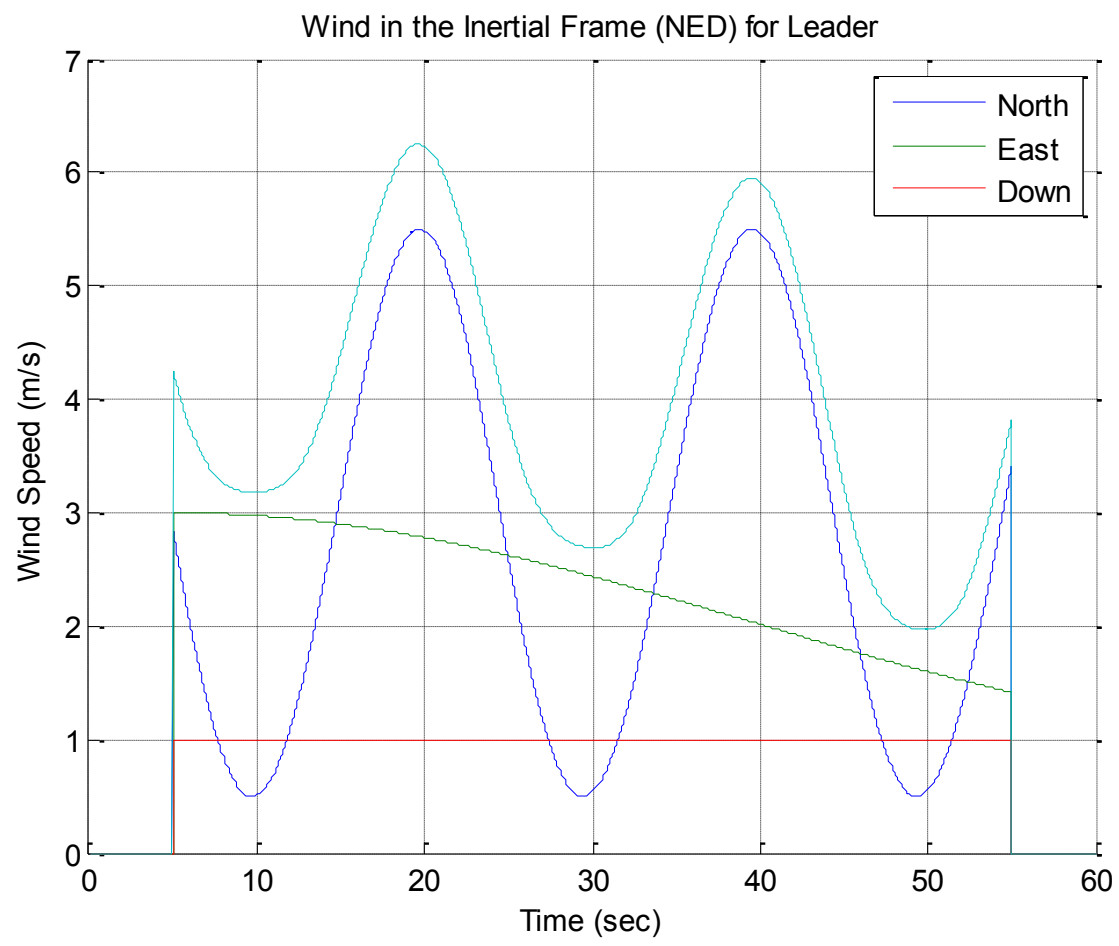

Figure 5.44 Wind field used for UKF wind estimation of leader UAV

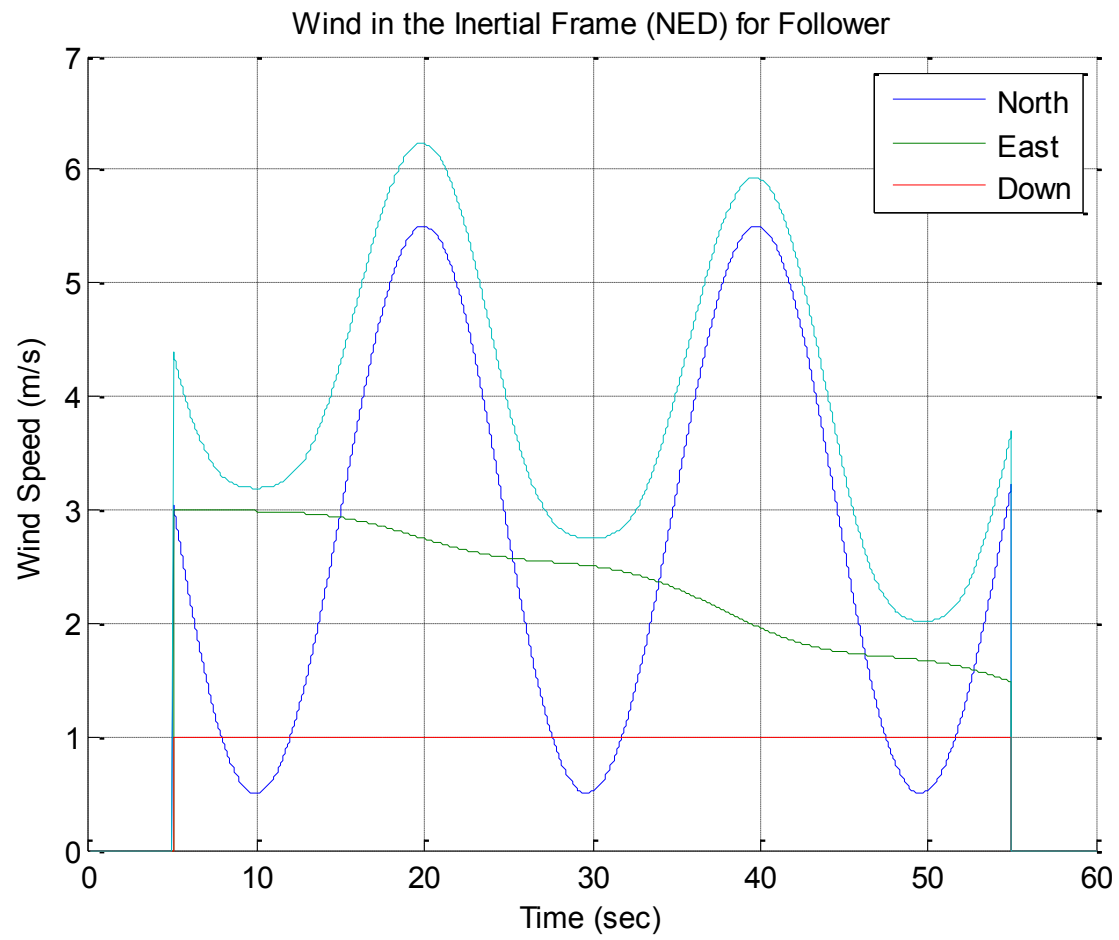

Figure 5.45 Wind field used for UKF wind estimation of follower UAV 


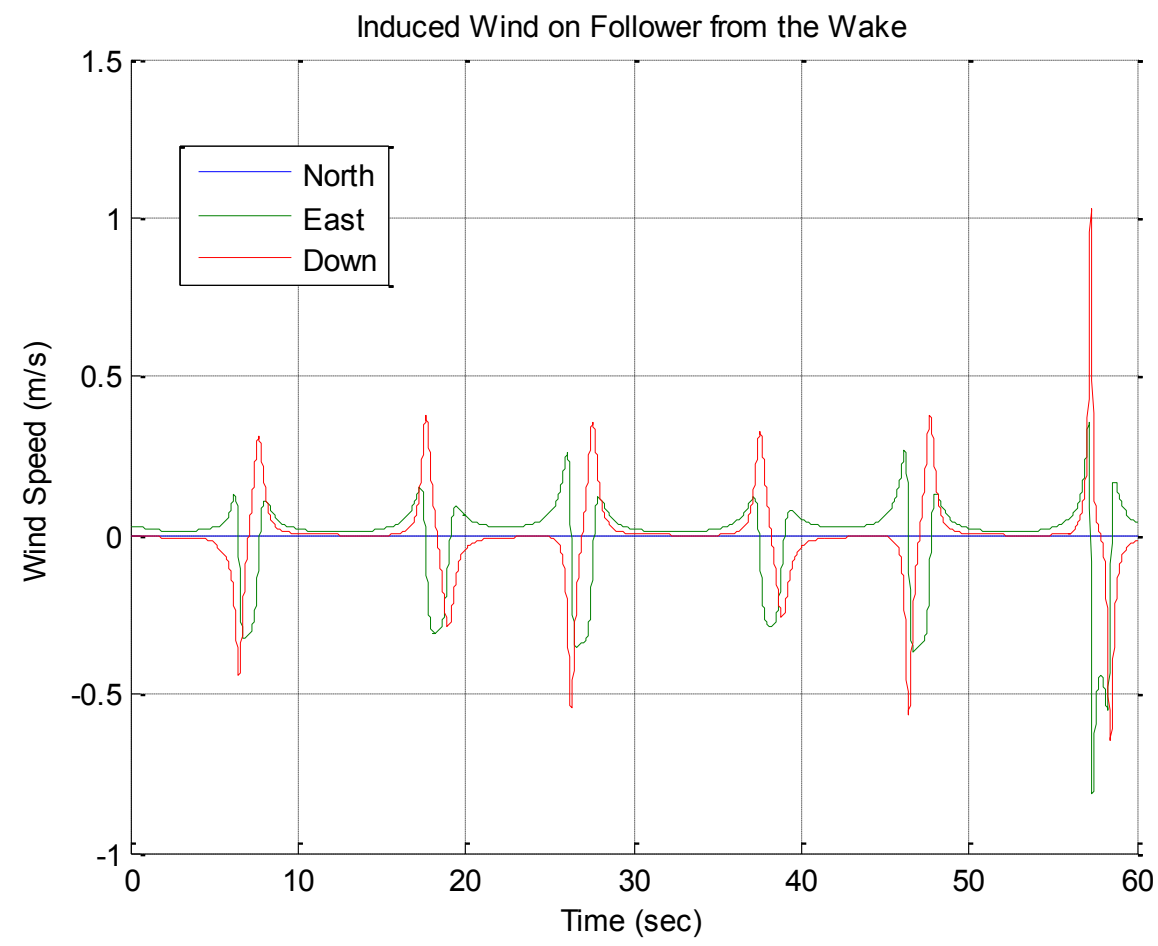

Figure 5.46 Induced wind on follower from the leader's wake

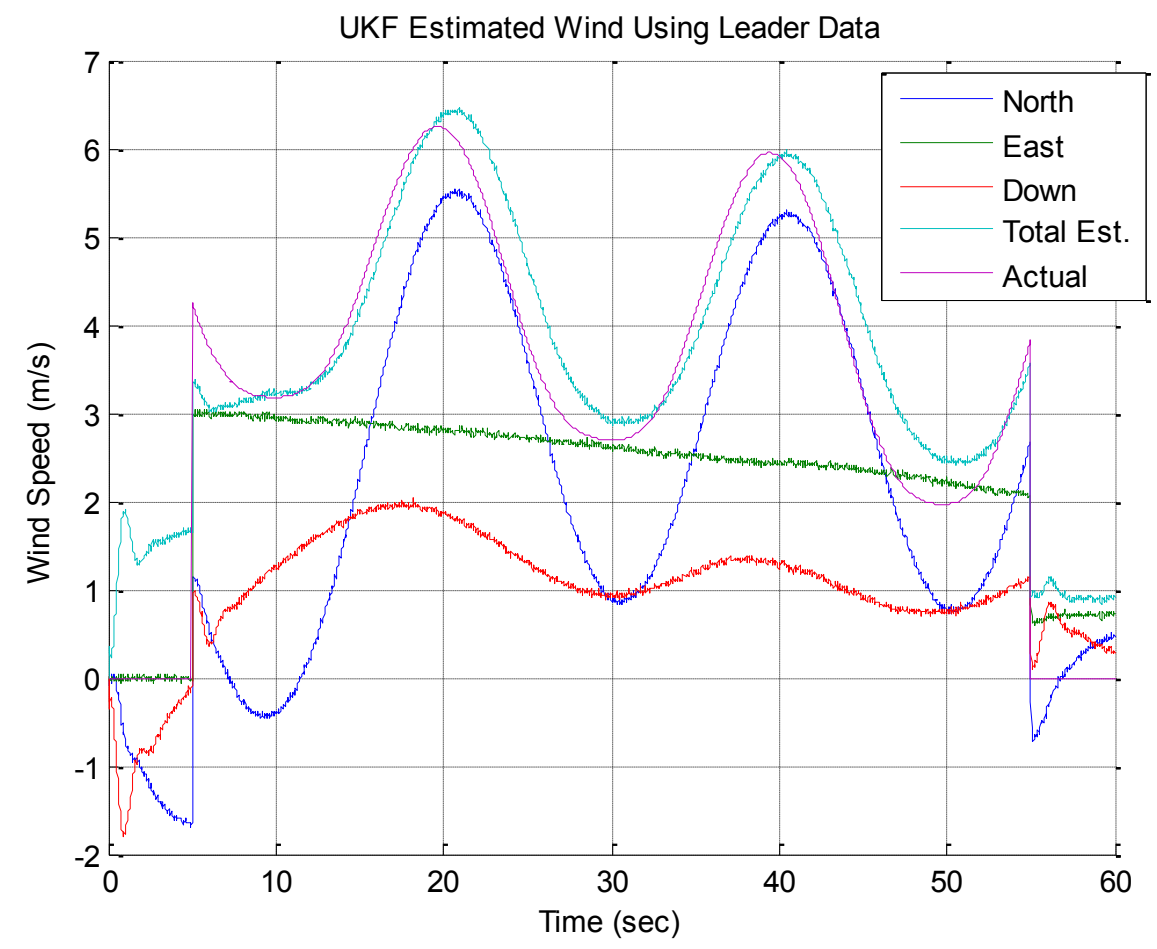

Figure 5.47 UKF wind estimation using flight data from leader only 


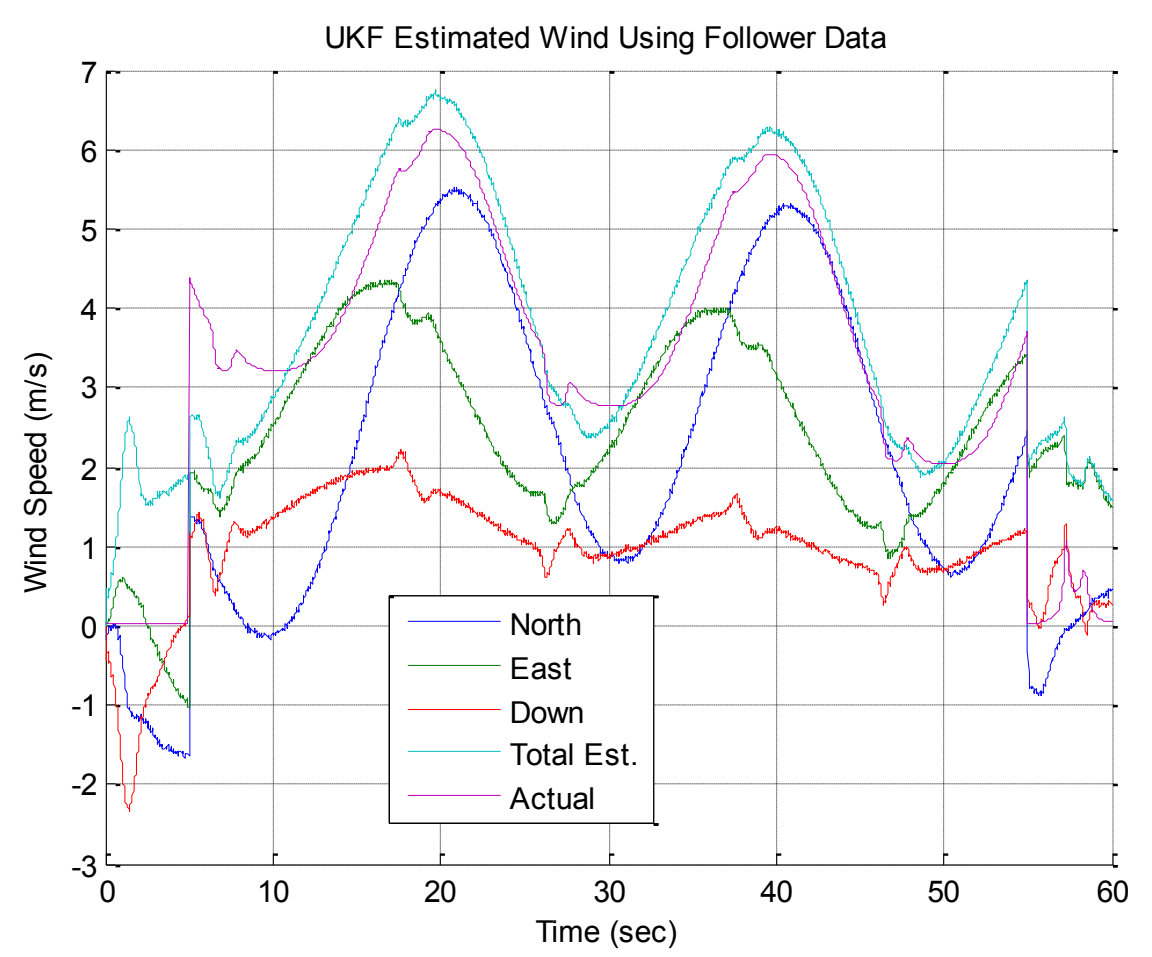

Figure 5.48 UKF wind estimation using flight data from follower only

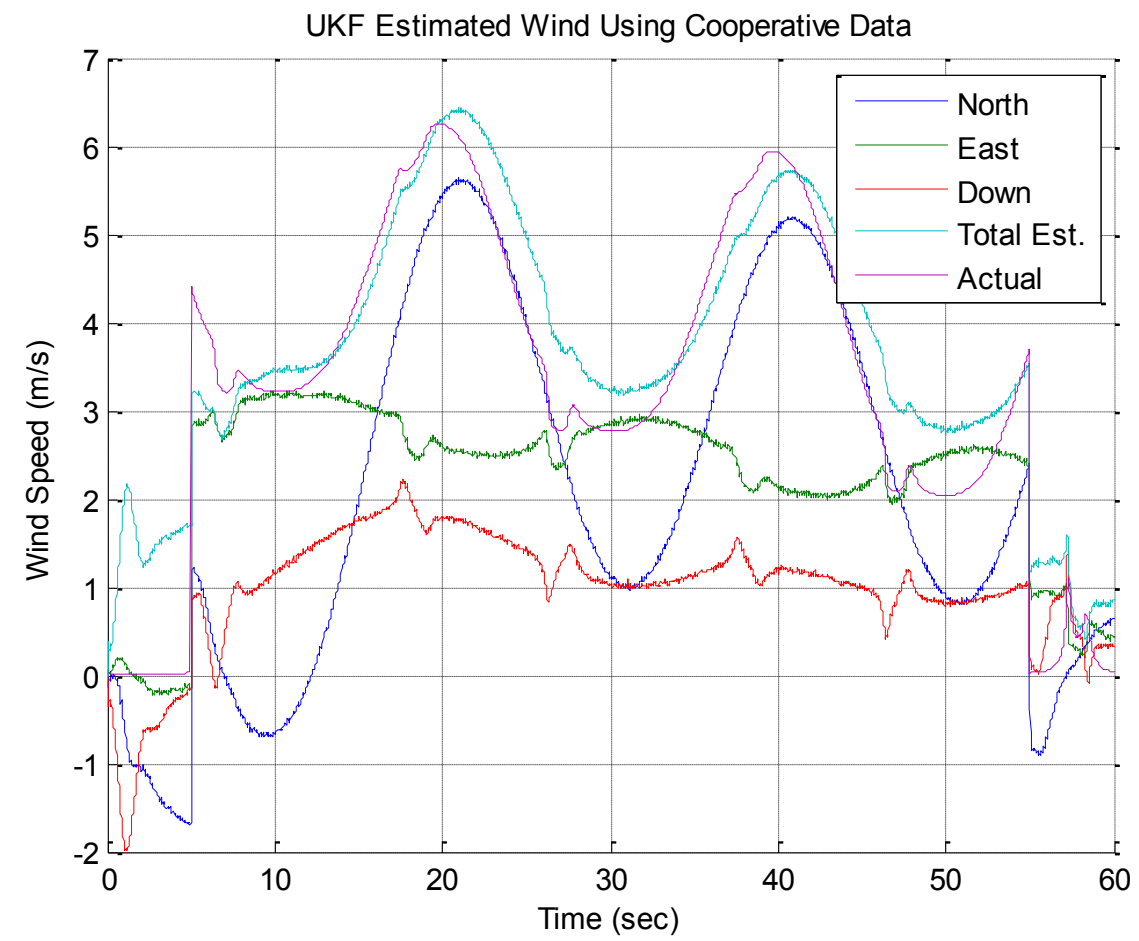

Figure 5.49 UKF wind estimation using cooperative wind estimation techniques 


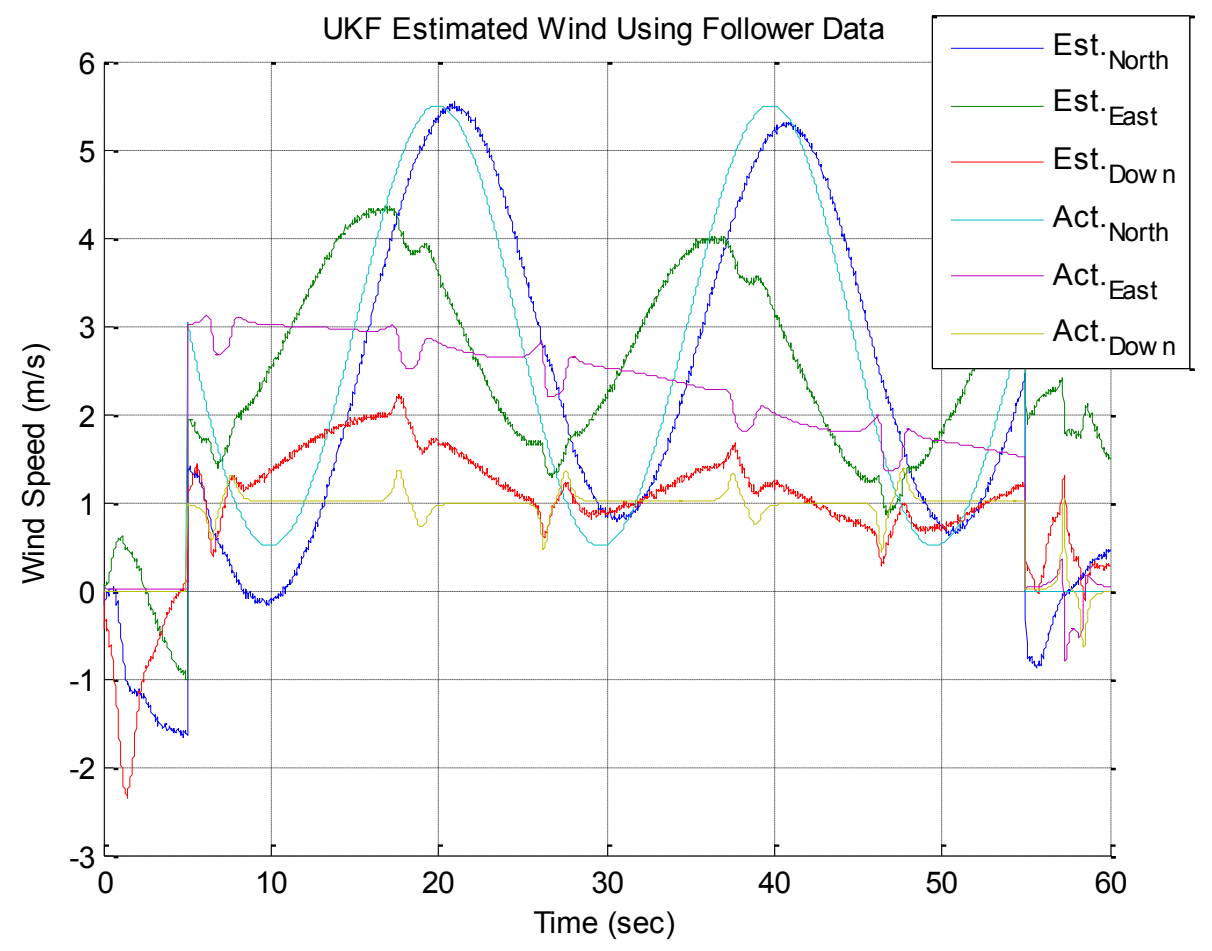

Figure 5.50 Wind states of UKF estimation using follower data

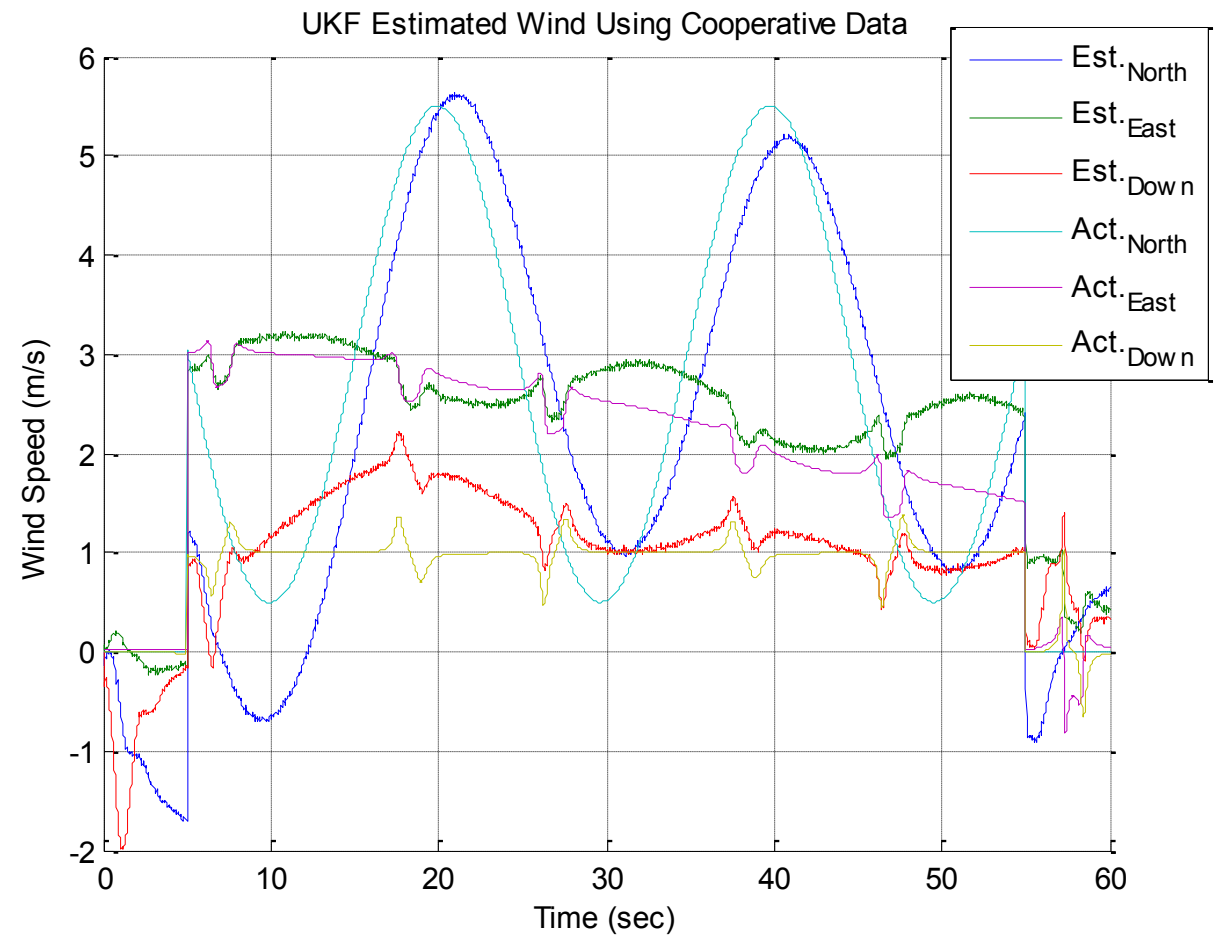

Figure 5.51 Wind states of UKF estimation using cooperative flight data 
Table 5.11 Cooperative wind estimation statistics

\begin{tabular}{lll}
\hline & Mean of Error $(\mathrm{m} / \mathrm{s})$ & Std of Error $(\mathrm{m} / \mathrm{s})$ \\
\hline Leader X & 0.6523 & 0.3809 \\
Leader $Y$ & 0.2718 & 0.2571 \\
Leader Z & 0.3757 & 0.3214 \\
\hline Leader Norm & 0.8003 & 0.5608 \\
\hline Follower X & 0.5958 & 0.3522 \\
\hline Follower Y & 0.9104 & 0.5857 \\
\hline Follower Z & 0.3751 & 0.3590 \\
\hline Follower Norm & 1.1509 & 0.7720 \\
\hline Coop X & 0.7569 & 0.4283 \\
Coop Y & 0.3282 & 0.2765 \\
\hline Coop Z & 0.3539 & 0.3290 \\
\hline Coop Norm & 0.8977 & 0.6068 \\
\hline
\end{tabular}




\section{Conclusion and Future Work}

\subsection{Conclusions}

This research set out to investigate wind estimation and wake modeling of small UAVs using a cost effective ADS, IMU, and GPS. This research can be used as better warning systems for both adverse weather conditions and wake encounters as well as used for energy harvesting through wind soaring and formation flight. Aside from these main topics of research a number of other areas of interest were investigated including the following:

1. Cooperative wind estimation where the estimated wind from the leader is used as a measurement for the follower's estimation algorithm

2. A comparison of using the proposed UKF for wind estimation to a more direct method of calculating the wind states

3. Gust alleviation in which the follower can control for gusts sensed by the leader in order to reduce the effects of gusts on aircraft accelerations

4. GCS development which included hardware and software development for GCS controlled flights as well as research into quality communication between the GCS and UAV

5. Wake visualization was conducted in order to physically validate the vortex model used in wake modeling for simulation and gust alleviation purposes

The research described here successfully showed through flight data the validity of using a UKF for wind estimation with a comparison to ground truth. Flight data was also used to sense the wake of a small UAV using an onboard ADS. Finally, wake visualization is shown using flight data which clearly shows the counter rotating vortex pair caused by the physics of the aerodynamics of flight.

Through modeling and simulation it was shown that cooperative wind estimation can increase the accuracy of wind estimation on the follower aircraft as it encounters both the global wind field and the leader's wake. Simulations have also shown that gust alleviation is possible through surface deflections that are proportional to the encountered gust. 


\subsection{Future Work}

Expanding on the research done here there are a number of areas that future research can be conducted. These include the following:

1. Cooperative wind and gust estimation during flight. This would allow for the simulation results to be validated and hopefully result in a wind estimation algorithm that exceeds current accuracy. Not only is this an interesting problem in itself but it will also benefit further research into gust alleviation control as well as energy harvesting through formation flight.

2. Wind gust alleviation control during formation flight. Simulations discussed previously have shown huge benefits can be achieved through simple surface deflections proportional to the magnitudes of the wind states and there derivatives. It would be interesting and beneficial to both manned and unmanned aviation if this type of gust suppression could be shown to work in actual flights. This type of research could lead to more comfortable flights for passengers which in turn mean safer flights because objects in the cabin will move around less.

3. Building on the ability of the follower to estimate the wind field as well as the induced wind from the leader's wake it would be worthwhile to investigate "sweet spot" flying under autonomous control. As discussed previously flying in the "sweet spot" of the leader quickly becomes a difficult task for human pilots and in some regions of the wake it is impossible to trim the aircraft for flight which increases the word load. Because of this autonomous control of the follower to fly within the sweet spot of the leader would drastically increase energy harvesting of UAVs and manned aircraft for long endurance flights. This challenge is also interesting because a new type of control would have to be introduced where instead of using aircraft states, which are effected by the wake, to fly in formation the controller would need to utilize the knowledge of the wind states for maximum benefit.

4. Another interesting area of research to build on this project would be to show the decrease in drag resulting from formation flight. This would help in autonomous control 
if the UAV could calculate its drag but it would also help to validate where the "sweet spot" is and to quantify its benefit.

5. One of the things mentioned in the section regarding the UKF in simulation data showed that as the UAV was flying at a different heading the filter converged quicker and its statistics were better. Given this information it would be beneficial to look into convergence of this UKF based on various heading directions and flight maneuvers. A study into the observability of 3D wind would help in understanding how to best implement and tune the UKF for the best possible wind estimation.

6. Finally, research into a 3D model of the wake would help to increase the fidelity of wake simulations as well as give valuable information to other areas of research such as "sweet spot" flight control and cooperative wind estimation. It has been shown through multiple research efforts as well as through the wake visualization discussed above that there is a component of the wake that moves along the $x$ axis of the aircraft which is not taken into account in the wake modeling used in this research. 


\section{Bibliography}

[1] D. Alexander, Nature's Flyers: Birds, Insects, and the Biomechanics of Flight. The Johns Hopkins University Press, 2002.

[2] I. Bajec and F. Heppner, "Organized flight in birds," Animal Behaviour, vol 78. iss. 4, pp 777-789, 2009.

[3] P. B. S., Lissaman, and C. A., Shollenberger, "Formation Flight of Birds," Science, Vol. 168, 22 May 1970, pp. 1003-1005.

[4] M. Allen and V. Lin, "Guidance and control of an autonomous soaring UAV," NASA Technical Memorandum TM-2007-214611, NASA Dryden Flight Research Center, 2007.

[5] D. Edward, "Implementation details and flight test results of an autonomous soaring controller," in Proceedings of the AIAA Guidance Navigation \& Control Conf., no. AIAA2008-7244, August 2008.

[6] K. Andersson, I. Kaminer, V. Dobrokhodov, and V. Cichella, "Thermal centering control for autonomous soaring: Stability analysis and flight test results," AIAA Journal of Guidance, Control, and Dynamics, vol. 49, no. 8, pp. 1292-1302, 2012.

[7] H. Chao and Y. Q. Chen, Remote Sensing and Actuation Using Unmanned Vehicles. Hoboken, NJ: Wiley-IEEE Press, 2012.

[8] D. Gray, "Using GPS to Accurately Establish True Airspeed." [http://www.ntps.edu/HTML/Downloads], 1998.

[9] S. McLaren, "Velocity estimate following air data system failure," Master's thesis, Air Force Institute of Technology, 2008.

[10] A. Cho, J. Kim, S. Lee, and C. Kee, "Wind estimation and airspeed calibration using a UAV with a single-antenna GPS receiver and pitot tube," IEEE Transactions on Aerospace and Electronics Systems, vol. 47, no. 1, pp. 109-117, 2011.

[11] R. Beard and T. McLain, Small Unmanned Aircraft: Theory and Practice. Princeton University Press, 2012.

[12] H. Palanthandalam-Madapusi, A. Girard, and D. Bernstein, "Wind field reconstruction using flight data," in Proceedings of the 2008 American Control Conference, pp. 18631868, June 2008.

[13] M. Kumon, I. Mizumoto, Z. Iwai, and M. Nagata, "Wind estimation by unmanned air vehicle with delta wing," in Proceedings of the IEEE Robotics \& Automation Conf., pp. 1896-1901, April 2005.

[14] D. Baird, R. Tolson, S. Bougher, and B. Steers, "Zonal wind calculations from mars global surveyor accelerometer and rate data," AIAA Journal of Spacecraft and Rockets, vol. 44, no. 6, pp. 1180-1187, 2007.

[15] D. Saban, J. Whidborne, and A. Cooke, "Simulation of Wake Vortex Effects for UAVs in Close Formation Flight," Aeronautical Journal, vol 113, iss. 1149, pp727-738, 2009. 
[16] W. Okolo and A. Dogan, "Determination of Sweet Spot for Trailing Aircraft in Formation Flight," AIAA Atmospheric Flight Mechanics Conference, Portland, 2011, AIAA 2011-6302.

[17] M. J. Vahon, R. J. Ray, K. R. Walsh, and K. Ennix, "F?A-18 Performance Benefits Measured During the Autonomous Formation Flight Project," NASA Dryden Flight Research Center September, 2003.

[18] J. Pahle, D. Berger, M. Venti, C. Duggan, J. Faber, and K. Cardinal, "An Initial Flight Investigation of Formation Flight for Drag Reduction on the C-17 Aircraft," AIAA Atmospheric Flight Mechanics Conference, Minneapolis, 2012.

[19] A. Kaden and R Luckner, "Modeling Wake Vortex Roll-Up and Vortex-Induced Forces and Moments for Tight Formation Flight," AIAA Guidance, Navigation, and Control Conference, Boston, 2013, AIAA 2013-5076.

[20] T. L., Jordan, J.V., Foster, R.M., Bailey, and C.M., Belcastro, “ AirSTAR: A UAV Platform for Flight Dynamics and Control System Testing" 25th AIAA Aerodynamic Measurement Technology and Ground Testing Conference, San Francisco, 2006, AIAA 2006-3307.

[21] B., Kayayurt, I., Yayla, A., Yapici, and C., Küçükoğuz, "Ground Control Station Avionics Software Development in ANKA UAV", IEEE/AIAA Digital Avionics Systems Conference (DASC), Seattle, vol., no., 2011, pp.5B6-1-5B6-7.

[22] "BEAR: Berkeley Aerobot Team," URL: http://robotics.eecs.berkeley.edu/bear/testbeds.html [cited 24 January 2013].

[23] D.E., Cox, K., Cunningham, and T., Jordan, "Subscale Flight Testing for Aircraft Loss of Control: Accomplishments and Future Directions," AIAA Guidance, Navigation, and Control Conference, Minneapolis, 2012, AIAA 2012-5029.

[24] "Generation IV Ground Control Station" URL: http://www2.I3com.com/uas/tech_uas/gen_iv.htm [cited 24 January 2013].

[25] K.A., Gluck, J.T., Ball, G., Gunzelmann, M.A., Krusmark, D.R., Lyon, and N.J., Cooke, “A Prospective Look at a Synthetic Teammate for UAV Applications" AIAA Infotech@Aerospace Conference, Arlington, 2005, AIAA 2005-6970.

[26] J., Franke, V., Zaychik, T., Spura, and E. Alves, "Inverting the Operator/Vehicle Ratio: Approaches to Next Generation UAV Command and Control." Association for Unmanned Vehicle Systems International and Flight International, Baltimore, 2005.

[27] I., Maza, F., Caballero, R., Molina, N., Peña, and A., Ollero, "Multimodal Interface Technologies for UAV Ground Control Station" Journal of Intelligent and Robotic Systems, Vol. 57, No. 1-4, January 2010, pp. 371-391.

[28] A.P., Tvaryanas, "Visual Scan Patterns During Simulated Control of an Uninhabited Aerial Vehicle (UAV)" Aviation, Space, and Environmental Medicine, Vol. 75, No. 6, June 2004, pp. 531-538. 
[29] R., Marsh, K., Ogaard, M., Kary, J., Nordlie, and C. Theisen, "Development of a Mobile Information Display System for UAS Operations in North Dakota," International Journal of Computer Information Systems and Industrial Management Applications, Vol. 3, 2011, pp. 435-443.

[30] Z., Merceruio, and K., Phillips, "Aerodynamic and Thrust Force Modeling for a Propulsion Assisted Control Aircraft Test Bed", AIAA Guidance, Navigation, and Control Conference 2011. Portland, OR. August, 2011.

[31] J. Gross, "Sensor Fusion Based Fault-Tolerant Attitude Estimation Solutions for Small Unmanned Aerial Vehicles," Doctor of Philosophy in Aerospace Engineering, Department of Mechanical and Aerospace Engineering, West Virginia University, 2011.

[32] G. Campa, "RTAl-Target HOWTO." Real Time Application Interface. [Online] [Cited: October 21, 2013.] www.rtai.org.

[33] M. Rhudy, J. Gross, Y. Gu, and M. Napolitano, "Fusion of GPS and Redundant IMU Data for Attitude Estimation," AIAA Guidance, Navigation, and Control Conference, Minneapolis, 2012, AIAA 2012-5030

[34] Z. Sarris, "Survey of UAV Applications in Civil Markets" IEEE Mediterranean Conference on Control and Automation, Croatia, 2001.

[35] H. Ranter, "Airliner Accident Statistics 2006," Tech. rep., Aviation Safety Network, 2007.

[36] S., Gururajan, A., McGrail, Y., Gu, B., Seanor, M., Napolitano, J., Prucz, and K., Phillips, "Identification of Aerodynamic Parameters for a Small UAV from Flight Data", 52nd Israel Annual Conference on Aerospace Sciences, Technion-I.I.T, Haifa, Israel, Feb 29 - Mar 1, 2012.

[37] M.O., Rauw, "FDC 1.2 - A Simulink Toolbox for Flight Dynamics and Control Analysis," 2nd ed., Haarlem, The Netherlands, 1997.

[38] Instrument Flying Handbook, U.S. Department of Transportation Federal Aviation Administration, Oklahoma City, 2001, Chap. 5.

[39] Atmospheric Flight Dynamics, Describing atmospheric turbulence, www.aerostudents.com viewed on Nov. 2, 2013.

[40] F. M. Hoblit, Gust Loads on Aircraft: Concepts and Applications, ISBN: 0-930403-45-2.

[41] Peet Bros., “Owner's Manual.", 3 Sept 2013 <http://www.peetbros.com/pdf/ULTIMETER2100Manual.pdf>

[42] M. Rhudy, T. Larrabee, H. Chao, Y. Gu, and M. Napolitano, "UAV Attitude, Heading, and Wind Estimation Using GPS/INS and an Air Data System", AIAA Guidance, Navigation, and Control Conference, Boston, 2013, AIAA 2013-5201

[43] J.K., Uhlmann, and S.K., Julier, "A New Extension of the Kalman Filter to Nonliner Systems," Int. Symposium, Aerospace/Defense Sensing Simulation and Controls. 
[44] J. Roskam, Airplane Flight Dynamics and Automatic Flight Controls, DAR corporation, Lawrence, KS. 2003, Chap. 1.

[45] J. Langelaan, N. Alley, and J. Neidhoefer, "Wind Field Estimation for Small Unmanned Aerial Vehicles," Journal of Guidance, Control and Dynamics, vol. 24, no. 4, pp. 1016-1030, 2011.

[46] U.S. Environmental Protection Agency, "Meterological Monitoring Guidance for Regulatory Modeling Applications," February, 2000.

[47] D., Hinton, and C. R., Tatnall, "A Candidate Wake Vortex Strngth Definition for Application to the NASA Aircraft Vortex Spacing System (AVOSS), NASA Technical Memorandum 110343.

[48] D., Burnham, and J., Hallock, Chicago Monostatic Acoustic Vortex Sensing System, Vol. IV. Wake Vortex Decay, DOT/FAA/RD-79-103 IV, July 1982.

[49] T., Gerz, F., Golzapfel, and D., Darracq, "Commercial Aircraft Wake Vortices," Progress in Aerospace Science, vol. 38, pp181-208, 2002.

[50] Y., Gu, G., Campa, B., Seanor, S., Gururajan and M.R., Napolitano (2009). Autonomous Formation Flight: Design and Experiments, Aerial Vehicles, Thanh Mung Lam (Ed.), ISBN: 978-953-7619-41-1, InTech, Available from: http://www.intechopen.com/books/aerial_vehicles/autonomous_formation_flight_design_and_experiments

[51] D., Tancredi, "Development of Adaptive Control Laws for Aircraft Actuator Fault Accommodation," Masters of Science in Aerospace Engineering, West Virginia University, 2011.

[52] K., Phillips, G., Campa, S., Gururajan, B., Seanor, M., Napolitano, Y., Gu., and M., Fravolini, "Parameter Identification for Application within a Fault-Tolerant Flight Control System," AIAA MST, Toronto, 2010.

[53] Stevens, and Lewis, "Aircraft Control and Simulation," 2nd ed. London, Springer-Verlag, 1995. 